



\title{
On the evolution and simulation of strange-mode instabilities
}

\author{
Dissertation \\ zur Erlangung des Doktorgrades \\ der Mathematisch-Naturwissenschaftlichen Fakultäten \\ der Georg-August-Universität zu Göttingen \\ vorgelegt von \\ Matthias Grott \\ aus Hamburg
}

Göttingen 2003 
D7

Referent: Prof. Dr. Wolfgang Glatzel

Korreferent: Prof. Dr. Gerald Warnecke

Tag der mündlichen Prüfung: 22.08.03 


\section{Danksagung}

Viele Menschen haben zum Gelingen dieser Arbeit beigetragen, und ich möchte mich an dieser Stelle bei ihnen bedanken. Aufgrund der großen Anzahl können unmöglich alle beteiligten Personen in dieser Danksagung Platz finden, doch möchte ich einige von ihnen hier namentlich erwähnen:

Herrn Prof. Dr. K.J. Fricke danke ich für die vielfältige Unterstützung und das Interesse, das er der vorliegenden Arbeit entgegen gebracht hat.

Mein besonderer Dank gilt Prof. Dr. W. Glatzel und Dr. Sergei Chernigovski, die mir alle wichtigen Kenntnisse über stellare Pulsationen und numerische Simulationen vermittelt haben. Sie haben meiner Arbeit großes Interesse entgegen gebracht und standen für Fragen immer zur Verfügung. Sergei Chernigovski danke ich für das zur Verfügungstellen der Grundversion des nicht-linearen Pulsationsprogrammes. Ferner wäre ohne den von Wolfgang Glatzel und Dr. Alfred Gautschy erstellten numerische Stabilitätsanalyse-Code ein Teil dieser Arbeit nicht möglich gewesen.

Herrn Prof. Dr. G. Warnecke danke ich für die Übernahme des Korreferats.

Ich bin insbesondere Jörg Huber, Ingo Berentzen und Dagmar Krefting für viele interessante Diskussionen zu Dank verpflichtet. Ingo Berentzen war ferner am Layout der vorliegenden Arbeit maßgeblich beteiligt. Außerdem haben alle drei immer versucht, meine Eigenheiten zu ertragen.

Mein Dank gilt auch den MitarbeiterInnen der Abteilung II der Universitäts Sternwarte. Durch das entspannte Arbeitsklima gingen viele Dinge leichter von der Hand. Besonders hervorheben möchte ich die aufopferungsvolle Tätigkeit unseres Kaffeewartes Thomas Lilly, ohne die diese Arbeit nicht möglich gewesen wäre.

Die numerischen Rechnungen wurden auf den Parallelrechnern der Gesellschaft für wissenschaftliche Datenverarbeitung Göttingen (GWDG) durchgeführt. Die deutsche Forschungsgemeinschaft hat die Arbeit mit einem Stipendium im Rahmen des Graduiertenkollegs "Strömungsinstabilitäten und Turbulenz" finanziell unterstützt. 



\section{Contents}

\section{Introduction}

1.1 Luminous stars and the Humphreys-Davidson (HD) limit . . . . . . . . 1

1.2 Instability mechanisms . . . . . . . . . . . . . . . . 4

1.3 Objectives of this study . . . . . . . . . . . . . 8

\section{Basic equations and methods}

\section{Stellar structure}

2.1 Equations of stellar structure . . . . . . . . . . . . . . . . . . 13

2.2 Stellar envelope models . . . . . . . . . . . . . . . 15

\section{Linear stability analysis}

3.1 Radial perturbation equations . . . . . . . . . . . . . . . 17

3.2 The work integral . . . . . . . . . . . . . . . . 21

3.3 Mode interaction . . . . . . . . . . . . . . . . . 22

3.4 Numerical method . . . . . . . . . . . . . . . . . 28

4 Non-linear evolution of instabilities

4.1 Relaxation of initial models . . . . . . . . . . . . . . . 31

4.2 Non-linear equations . . . . . . . . . . . . . . . . . . 32

4.3 Numerical method . . . . . . . . . . . . . . . . 33

\section{Results}

\section{Luminous blue variables}

5.1 Hydrostatic envelope models . . . . . . . . . . . . . . . . . 39

5.2 Linear stability analysis . . . . . . . . . . . . . . . . . . . . . . 42

5.3 Non-linear evolution of instabilities . . . . . . . . . . . . . 45

\section{Captured shocks}

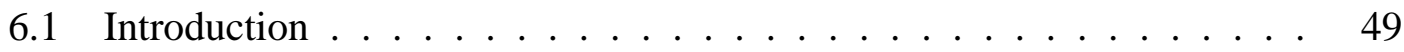


6.2 Basic assumptions and methods ............... 50

6.3 Numerical results . . . . . . . . . . . . . . . . . . 53

6.4 An analytical model . . . . . . . . . . . . . . . . . 61

6.5 Conclusions . . . . . . . . . . . . . . . . 71

7 Domain decomposition

7.1 Introduction . . . . . . . . . . . . . 76

7.2 Basic equations and assumptions . . . . . . . . . . . . 77

7.3 Domain decomposition . . . . . . . . . . . . . . . . . . 78

7.4 Application to a P Cygni model . . . . . . . . . . . . . . . . 88

7.5 Conclusions . . . . . . . . . . . . . . . . . . 95

\section{Grid reconstruction}

8.1 Introduction . . . . . . . . . . . . . . . . . . . . 999

8.2 Basic equations and assumptions . . . . . . . . . . . . . . 100

8.3 Grid reconstruction . . . . . . . . . . . . . . . . . . 104

8.4 Conclusions . . . . . . . . . . . . . . . . . . 113

\section{Summary}

9.1 Summary of results . . . . . . . . . . . . . . . . . 117

9.2 Conclusions . . . . . . . . . . . . . . . . . . . . . . . . 119

9.3 Future research . . . . . . . . . . . . . . . 120

\section{Appendix}

A On a criterion for adiabatic instability

B The work integral 


\section{Introduction}

\subsection{Luminous stars and the Humphreys-Davidson (HD) limit}

One of the open questions in stellar evolution theory is the explanation of the so called Humphreys-Davidson limit (Humphreys \& Davidson 1979), a luminosity limit in the Hertzsprung-Russel diagram (HRD), above which no stable stars are observed. For high temperatures this limit is temperature dependent, whereas for cooler supergiants it is independent of temperature. Figure 1.1 shows the upper part of the observed HRD for the Galaxy and the Large Magellanic Cloud, indicating the HD limit by a solid line. Empirically, it implies the following relation between luminosity and temperature (Humphreys \& Davidson 1979):

$$
\begin{aligned}
& \log \frac{L}{L_{\odot}}=5.42+2.34 \log \frac{T_{\text {eff }}}{10^{4} K} \quad \text { for } \quad 15000 K \leq T_{\text {eff }} \leq 30000 K \\
& \log \frac{L}{L_{\odot}}=5.8 \quad \text { for } \quad 3000 K \leq T_{\text {eff }} \leq 15000 K
\end{aligned}
$$

where $L_{\odot}=3.85 \cdot 10^{33} \mathrm{erg} \mathrm{sec}^{-1}$ is the solar luminosity and $L$ and $T_{\text {eff }}$ are the luminosity and effective temperature, respectively. In the vicinity of the temperature dependent part of the HD limit a particularly interesting class of luminous blue supergiants having temperatures $T_{\text {eff }}$ between $8000 \mathrm{~K}$ and $27000 \mathrm{~K}$ and luminosities of $\frac{L}{L_{\odot}} \approx 10^{6}$ is encountered. These so called luminous blue variables (LBVs) or S Dor stars may be characterised as follows (Humphreys R.M. \& Davidson K.1994).

LBVs show photometric variability over a wide range of amplitudes and time scales. The most spectacular variations are giant eruptions during which the star can become brighter by more than $2^{\mathrm{m}}$ in the visual band. Examples for giant eruptions are $\eta$ Car's dramatic outburst from 1837 to 1860 and P Cyg's behaviour around 1600. After these major outbursts the stars may fade rapidly and remain relatively quiescent for long periods. Although the timescales for the giant eruptions remain uncertain, hundreds to thousands of years seem to be reasonable estimates for the frequency of these events. During eruptions, the luminosity of the objects stays approximately constant, although a variation of one magnitude in bolometric brightness was reported for the famous $\eta$ Car eruption (Davidson 1988). Figure 1.2 gives some examples for observed eruptions of LBVs in the HR diagram. Dashed lines indicate the transitions from quiescence (maximum temperature) to visual maximum (minimum temperature) at constant luminosity. 

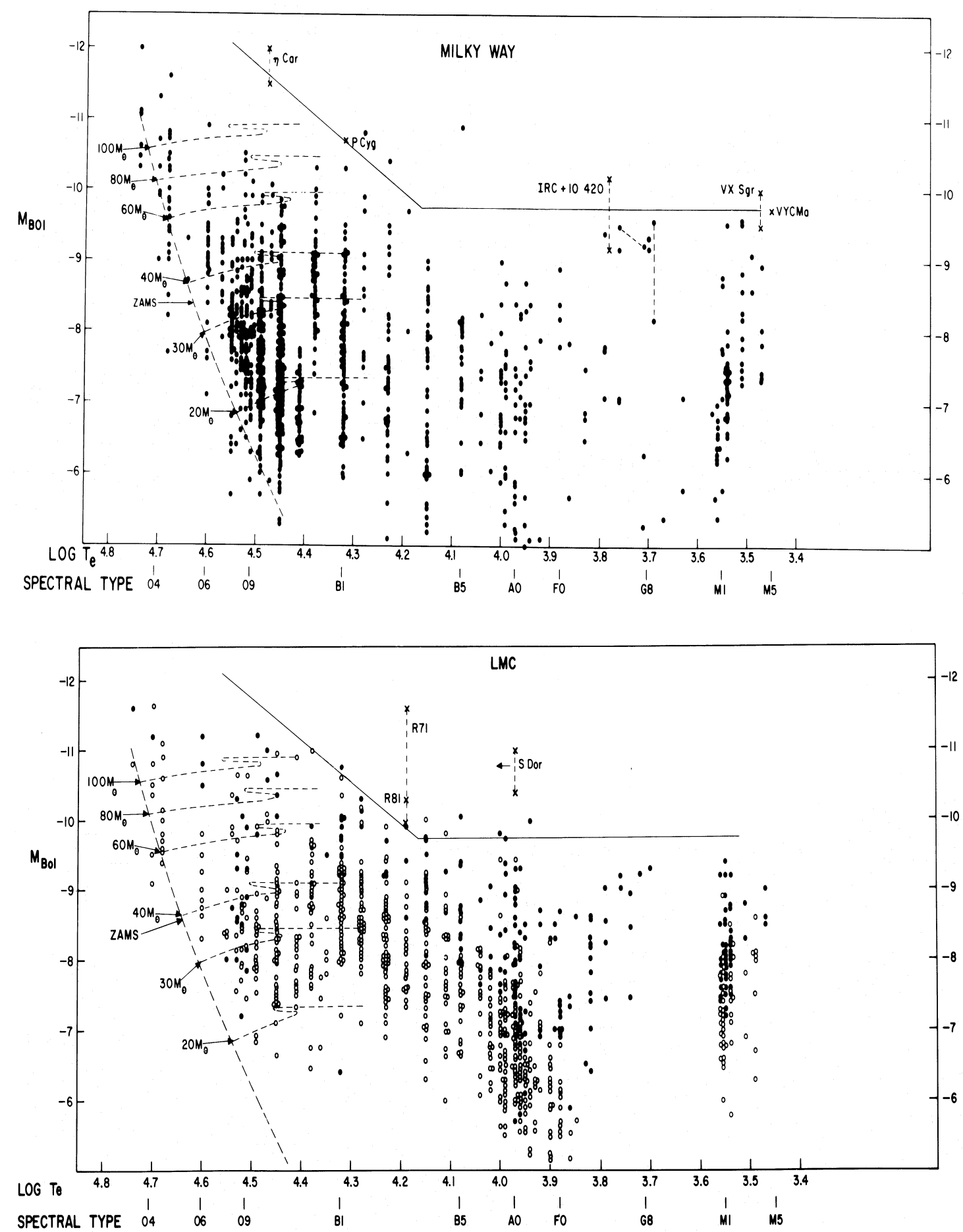

Figure 1.1: The Hertzsprung-Russel diagram for the galactic and LMC supergiants. The position of the ZAMS and selected evolutionary tracks with mass loss are shown as dashed lines. The solid line defines the approximate upper boundary of the supergiant luminosities. The positions of the peculiar stars $\eta$ Car and P Cyg in the Milky Way and $\mathrm{S}$ Dor in the LMC are indicated. Both figures are taken from Humphrey and Davidson, 1979. 


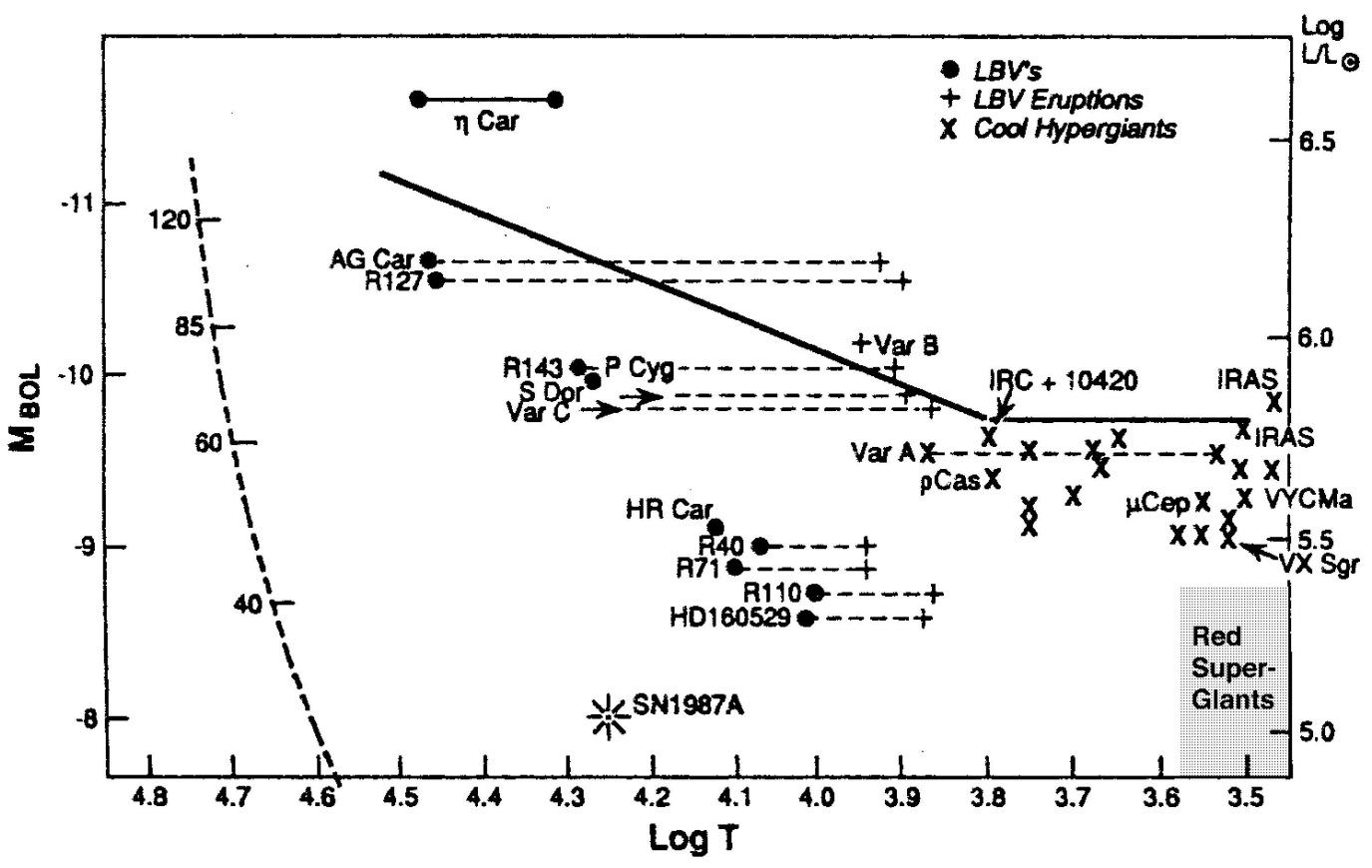

Figure 1.2: A schematic HR diagram for the most luminous stars. The dashed lines represent the LBV transition from quiescence ( $\bullet$ ) to the eruption (+) stage. The most luminous cool supergiants are also plotted. The empirical HD limit is shown as a solid line. The figure is taken from Humphreys and Davidson, 1994.

Smaller eruptions of $1-2^{\mathrm{m}}$ are observed on time scales of 10-40 years and might even look semi regular at times. At visual maximum, the stars atmosphere is considerably expanded, while the luminosity remains practically unchanged. The visual brightening is caused by a shift of the emission from ultraviolet to visual wavelengths. Superimposed on these long-term normal eruptions are smaller oscillations of about half a magnitude, acting on the time scale of months to a few years. In addition, microvariations of $\leq 0.1^{\mathrm{m}}$, which have also been reported for normal supergiants, have been observed for individual LBVs.

Associated with the LBV eruptions are high mass loss rates of typically $10^{-5}-10^{-4}$ $M_{\odot} \mathrm{yr}^{-1}$, being 10-100 higher than those of normal supergiants with comparable luminosities. If one includes the giant eruptions in this estimate, the mass loss rates can be as high as $10^{-2} M_{\odot} \mathrm{yr}^{-1}$ (Davidson 1988).

Most LBVs show some evidence for circumstellar ejecta. In the case of $\eta \mathrm{Car}$ it is even opaque enough to obscure the star and clearly visible as the "homunculus" nebula (see figure 1.3). Usually the presence of a ring nebula or circumstellar shell produced by the high mass loss is more common. Spectroscopic analyses of the ejecta show that they are nitrogen and helium enriched, i.e., presumably CNO-processed material has brought to the surface by mixing and mass loss, implying that LBVs are evolved stars.

To summarise, an LBV may be defined as an evolved, very luminous hot supergiant 


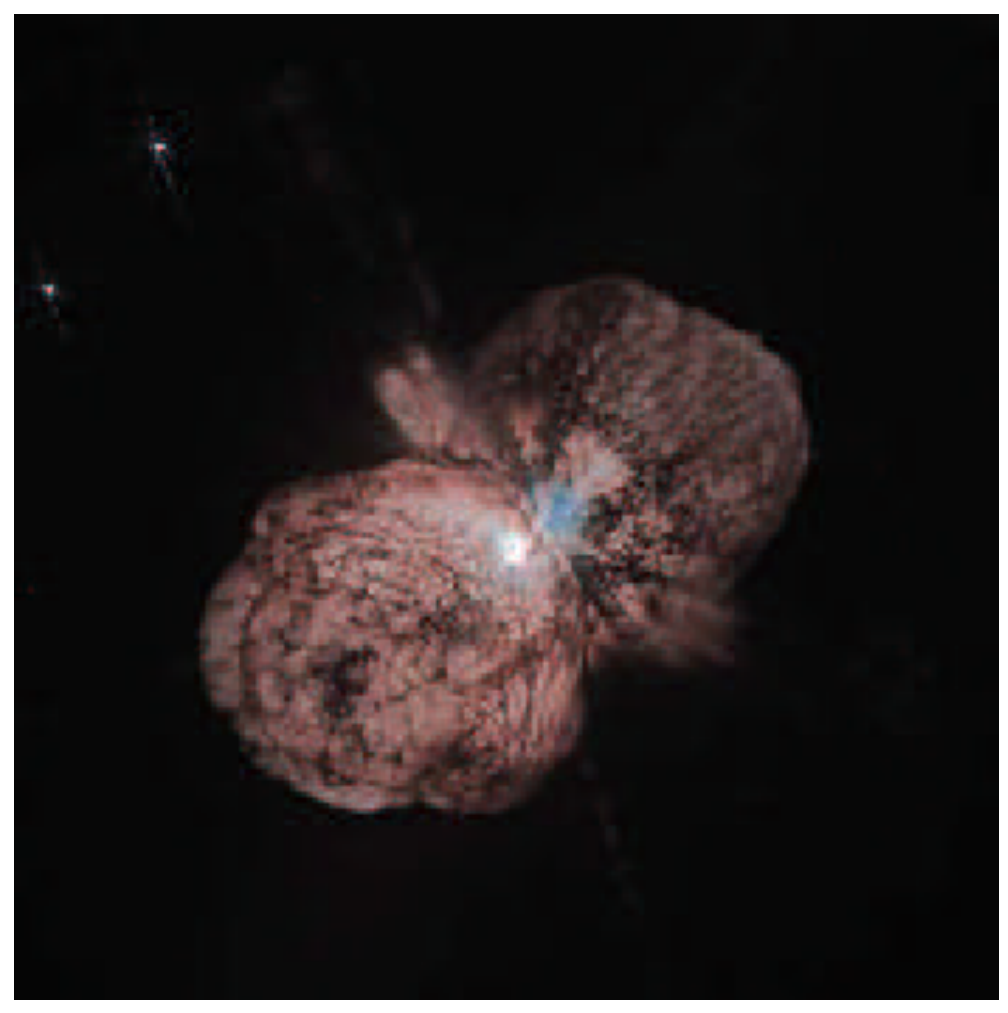

Figure 1.3: Image of the homunculus nebula surrounding the supergiant $\eta$ Car, as taken by the HST (Jon Morse, University of Colorado, and NASA).

which suffers irregular eruptions like, e.g., S Dor and AG Car, or more rarely giant eruptions as $\eta$ Car and P Cyg. Eruptions and variability are usually attributed to an instability which results in a considerably enhanced averaged mass outflow, leading to the formation of an expanded atmosphere at visual maximum. The visual brightness variations are caused by a shift in the stars spectral energy distribution. Many mechanisms for the instability driving the mass outflow have been discussed in the past, but no consensus about the correct interpretation has been reached so far.

\subsection{Instability mechanisms}

The first instability mechanism envisioned for these stars was the $\epsilon$-mechanism (Ledoux 1941, Schwarzschild \& Harm 1959). This mechanism is energised in the stellar core and depends on nuclear reactions. According to Ziebarth (1970), the mass above which stars become unstable with respect to the $\epsilon$-mechanism lies at $94 M_{\odot}$. However, this process is strongly damped by internal composition gradients. Therefore evolved stars like LBVs are unlikely candidates for this instability. Studies of its non-linear evolution by Appenzeller (1970) remain inconclusive since the amplitude of the pulsation was 
enhanced in this simulation by artificial energy input.

De Jager (1984) proposed that the instabilities might be caused by strong turbulent pressure gradients in the convective zones of the envelope. However, this process is only efficient for temperatures below $6000 \mathrm{~K}$, since for higher temperatures the relative contribution of convection to the luminosity amounts to at most one per cent and the contribution of the turbulent pressure to the total pressure reaches at maximum $10^{-6}$.

Other instability mechanisms that have been discussed are connected to the high intrinsic luminosities of LBVs, which are close to the so called Eddington limit. This is an upper limit to the luminosity to mass ratio for a static stellar envelope. The Eddington factor is usually defined by

$$
\Gamma=\frac{\kappa L}{4 \pi c G M}
$$

where $c$ is the speed of light, $G$ the gravitational constant, $L$ the luminosity and $M$ the mass of the star. It is calculated considering only the contribution of electron scattering to the opacity $\kappa$. If $\Gamma$ exceeds unity, the acceleration due to radiation pressure exceeds the gravitational acceleration and the star can no longer stay in hydrostatic equilibrium. Typically, LBVs have Eddington factors of $\sim 0.5$ or higher. Motivated by this fact, Davidson (1988) and Appenzeller (1988) have proposed a modified Eddington limit, i.e., one in which the opacity is temperature dependent. Due to an opacity maximum around $T \approx 15000 K$, caused by singly ionised elements of the iron group, the luminosity can exceed the Eddington luminosity locally thus resulting in $\Gamma \geq 1$, which is thought to destabilise the star. However, LBVs with effective temperatures up to $\approx 35000 K$ have been observed.

An explanation in the same direction was proposed by Langer (1998). He argued that stellar rotation can reduce the Eddington luminosity considerably by including the centrifugal acceleration into the force balance which reads

$$
g_{\text {total }}=g_{\text {grav }}+g_{\text {rot }}+g_{\text {rad }}
$$

The maximum radiative acceleration is reduced by the centrifugal acceleration. However, in his approach he assumed the radiation field to be spherically symmetric, even in the presence of stellar rotation. As Glatzel (1998) pointed out, this contradicts von Zeipels theorem of gravity darkening (von Zeipel 1924, Tassoul 1978), which predicts vanishing flux at the equator for critical rotation. Langer (1999) argued that according to a generalised von Zeipels theorem derived by Kippenhahn (1977), stellar rotation may either enhance or decrease the radiative flux on the equator, depending on the internal rotation law. However, to our knowledge, no significant deviations from the classical von Zeipels theorem have been reported.

Other mechanisms invoke instabilities located below the photosphere of the LBVs. The famous $\kappa$-mechanism, that drives the $\delta$ Cephei pulsations, has also been proposed as an explanation for LBVs by Moskalik and Dziembowski (1992). However, this has not been verified (cf. Glatzel \& Kiriakidis 1993b, Stothers \& Chin 1993). 
In his "geyser model", Maeder $(1988,1992)$ claims that subphotospheric density inversions are responsible for instability and variability in LBVs. They are connected to the convection zones in the stellar envelope and caused by a negative entropy gradient caused by inefficient convection, which can be expressed as

$$
\frac{\partial s}{\partial r} \propto \frac{1}{u_{s}^{2}} \frac{\partial p}{\partial r}-\frac{\partial \rho}{\partial r}
$$

where $u_{s}$ is the local sound speed, $p$ the pressure and $\rho$ the density. If the (negative) pressure gradient is not big enough, a positive density gradient, and hence a density inversion, is needed to provide the negative entropy gradient prescribed by the theory of convection adopted. Since a deviation from adiabatic behaviour is needed to have a significant negative entropy gradient in convection zones, this effect is only observed in the outer layers of stellar envelopes.

In incompressible fluids, density inversions lead to Rayleigh-Taylor instabilities, which Maeder claims to cause the observed LBV behaviour. However, density inversions are not only observed in the envelopes of LBVs, but also in a variety of other "normal" stars, which according to Maeder, should then also be unstable. This is not the case. Physically, the Rayleigh-Taylor instability has the same origin as the convective instability, namely a negative entropy gradient, but with the additional assumption of incompressibility. It seems therefore inconsistent to examine a convective stratification with respect to Rayleigh-Taylor instabilities. Therefore, it is questionable, if density inversions play an important role in the explanation of the LBV phenomenon.

Stothers and Chin $(1993,1994)$ argued that dynamical instabilities could be responsible for the observed outbursts in LBVs. They assume adiabatic changes of state and that the stellar envelope is acoustically separated from the core of the star by the opacity peak due to the contribution of heavy elements around $T=200000 K$. On this basis they derive the approximation

$$
\sigma^{2} \approx \frac{\left(3\left\langle\Gamma_{1}\right\rangle-4\right) \int_{r^{*}}^{R} p \mathrm{~d}\left(r^{3}\right)}{\int_{r^{*}}^{R} \frac{1}{3} r^{2} \rho \mathrm{d}\left(r^{3}\right)}, \quad\left\langle\Gamma_{1}\right\rangle=\frac{\int_{r^{*}}^{R} \Gamma_{1} p \mathrm{~d}\left(r^{3}\right)}{\int_{r^{*}}^{R} p \mathrm{~d}\left(r^{3}\right)}
$$

for the lowest eigenfrequency $\sigma$ of the stellar pulsation spectrum (Stothers 1999b). $\Gamma_{1}$ is the adiabatic index, $R$ the stellar radius and $r^{*}$ denotes the bottom of the stellar envelope. Highly questionable in this approach is the neglect of a surface term of comparable order to the integrals (see appendix A). If the mean adiabatic index $\left\langle\Gamma_{1}\right\rangle$ drops below $\frac{4}{3}, \sigma^{2}$ becomes negative, which corresponds to instability. Since in radiation pressure dominated envelopes as, e.g., the envelopes of LBVs, the adiabatic index $\Gamma_{1}$ is close to $\frac{4}{3}$, the value of $\Gamma_{1}<\frac{4}{3}$ in the ionisation zones of $\mathrm{H}$ and $\mathrm{He}$ is sufficient to provide a mean adiabatic index of $\left\langle\Gamma_{1}\right\rangle<\frac{4}{3}$. Using this approach, Stothers (1999a) analysed the envelopes of prominent LBVs and found them to lie well within the realm of dynamical instability.

However, Glatzel and Kiriakidis (1998) argued, that the adiabatic approximation is invalid for the considered stellar models and the non-adiabatic analysis should be used 


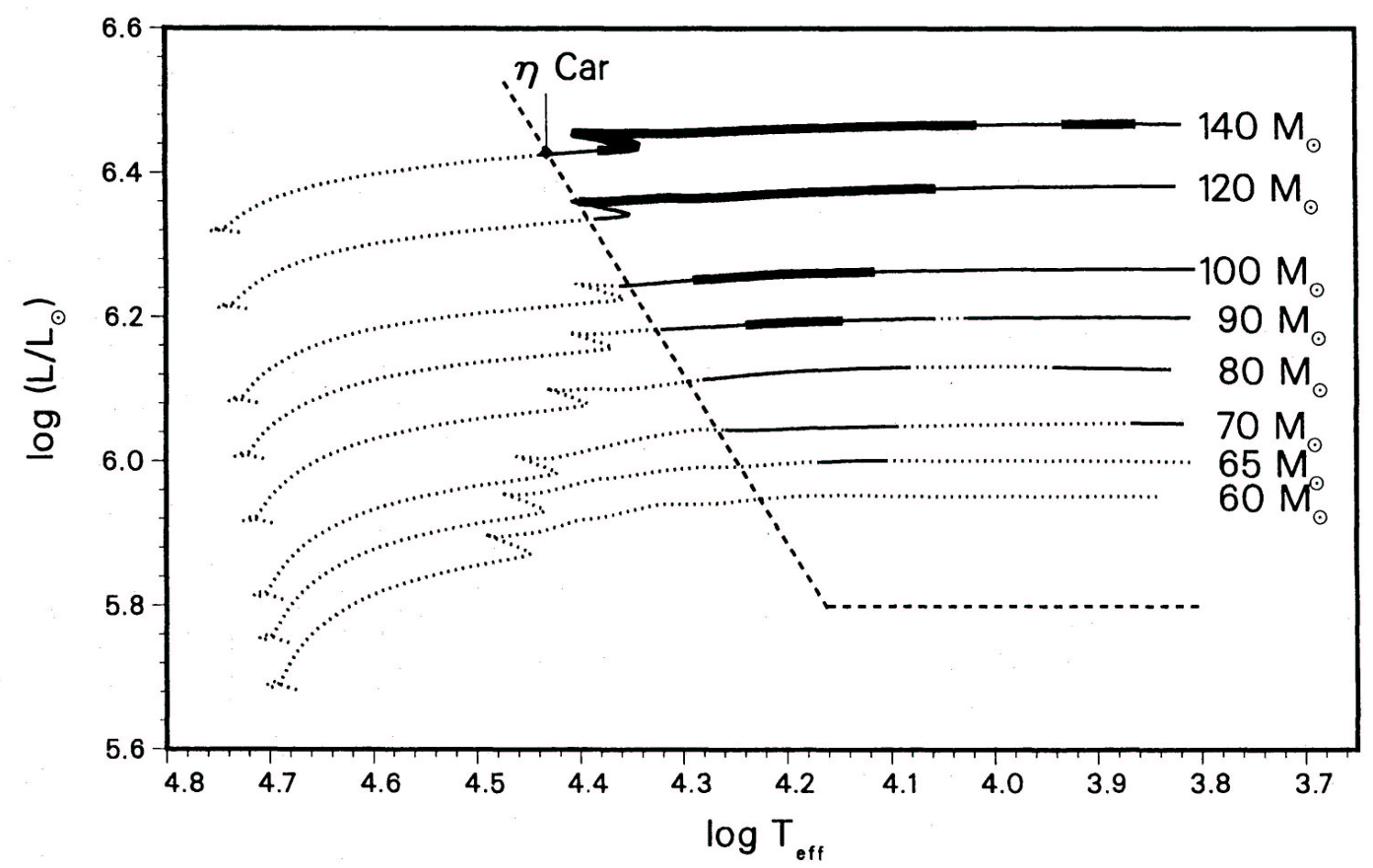

Figure 1.4: HR diagram containing the evolutionary tracks of eight stars (dotted lines) with the initial chemical composition $(X, Y, Z)=(0.746,0.25,0.004)$ and the initial masses indicated. Unstable phases are denoted by solid lines, and thick lines correspond to dynamical growth rates $\left|\sigma_{i}\right|>0.1$ in dynamical units. Together with the observed position of $\eta$ Car the location of the HD limit is shown as a dashed line. The figure is taken from Kiriakidis, Fricke \& Glatzel, 1993.

instead. Furthermore, the assumption that the stellar envelope is completely dynamically isolated from the stellar core seems questionable. Rather, the whole star must be tested for dynamical instability. In particular, the fundamental mode remains largely unaffected by the inversion of the sound speed around $T=200000 \mathrm{~K}$ and therefore the assumptions made do not hold. The neglect of the surface term is a severe error in the calculation. Finally, repeating the analysis of Stothers and Chin $(1993,1994)$, Glatzel and Kiriakidis (1998) did not find any dynamical instability. To summarise, this approach suffers from physical misconcepts (adiabatic approximation) and severe errors (surface terms) which casts doubt on the suggestion that dynamical instabilities play an important role in the explanation of the LBV phenomenon.

When investigating the stability of extreme helium stars, Gautschy and Glatzel (1990a) found unexpected new modes in the stellar pulsation spectrum which were called "strange modes". These modes provide resonances among the acoustic modes thus leading to instabilities. They are associated with growth rates in the dynamical regime. Subsequently they have also been identified in a variety of other stars, including Wolf-Rayet stars (Glatzel, Kiriakidis \& Fricke 1993) and massive stars (Glatzel \& Kiriakidis 1993a). Furthermore, they are not limited to spherical symmetry (Glatzel \& Kaltschmidt 2002, 
Glatzel \& Mehren 1996). Kiriakidis et al. showed (1993), that the boundary of the domain in the Hertzsprung-Russel diagram above which all stellar models are unstable irrespective of their metallicity -, coincides with the observed HD limit. As an example, the results of their linear stability analysis of stellar models with initial chemical composition $(X, Y, Z)=(0.746,0.25,0.004)$ is shown in figure 1.4 . Solid lines in the diagram correspond to unstable phases, thick lines to those with extremely high growth rates ( $\left|\sigma_{I}\right|>0.1$ in dynamical units). The observed HD-limit is shown as a dashed line. For stars with higher metallicity the instability region covers the whole range above the HDlimit and can even extend down to the main sequence. From this point of view, strange mode instabilities may therefore be suspected to be related to the LBV phenomenon.

\subsection{Objectives of this study}

This work is dedicated to the study of strange mode instabilities in the non linear regime. To this end, we have to construct stellar envelope models of LBVs having stellar parameters that fall into the instability domain of the HR diagram identified by Kiriakidis et al. (1993). Using the Riccati method (Gautschy \& Glatzel 1990b), we shall verify in a second step that these models suffer from multiple strange modes instabilities.

Then, the evolution of the instabilities will be followed into the non linear regime by direct numerical simulations. Strange mode instabilities, which act on the dynamical timescale, generate sound waves, which travel outwards and steepen to form shock waves with the result of global stellar pulsation.

For the models considered, a new phenomenon has appeared: After several pulsations a shocks is captured in the hydrogen ionisation zone and starts to oscillate on timescales much shorter than the dynamical one. Therefore, the question has to be addressed, whether the rapid shock oscillations observed in the numerical simulations are of physical origin, or numerical artifacts. If they are found to be of physical origin, an adequate numerical treatment of the different timescales involved has to be developed, as the rapid shock oscillations limit the integration time-step appreciably, preventing a long term study of the model.

For selected models, the strange mode instability was found to transfer mass from the inner region of the stellar envelope into its outer parts, which, due to the Lagrangian description adopted, leads to a reduced resolution in the instability region. As a consequence, the driving instability is suppressed and the mass flow out of the instability domain ceases. Should the mass flow observed in the early phases of the evolution of the instabilities indicate an outburst associated with mass flow of the object, this numerical problem would be crucial and ultimately provide a wrong answer to the basic question, whether strange mode instabilities are related to the LBV phenomenon. This calls for a solution of the resolution problem in the instability region. For this purpose a grid reconstruction algorithm will be developed.

This study consists of the following stages: In the first part, the basic equations and methods will be presented. In particular, the construction of initial models, their linear 
stability analysis and the non linear simulation of the instabilities will be discussed in general there. In the second part, these methods will be applied to models for LBVs including a review of their stability properties and the non linear evolution of strange mode instabilities they are suffering from. Subsequently, three research papers are presented, which address the following questions: The first paper investigates the origin of rapid shock oscillations, the second paper is dedicated to the adequate numerical treatment of the different timescales involved in their numerical simulation. The third paper deals with grid reconstruction required by the mass flow out of the instability domain caused by the instability itself. A summary together with a discussion of the presented results follows. 


\section{Part I}

\section{Basic equations and methods}





\section{Stellar structure}

\subsection{Equations of stellar structure}

In this section the equations of stellar structure are presented. For the present study, rotation and magnetic fields are neglected and we restrict ourselves to a spherically symmetric configuration. Appropriate for this situation is the Lagrangian description of hydrodynamics, i.e., the time $t$ and the mass $m$ inside a sphere of radius $r$ are the independent variables. The dependent variables are the radius $r$, the pressure $p$, the density $\rho$, the temperature $T$ and the luminosity $L$.

\subsubsection{Mechanical equations}

In the Lagrangian description mass conservation implies that the mass $m$ inside a sphere of radius $r$ is given by to the integral of the density $\rho$ over the volume of the sphere. In differential form this may be expressed as (see, e.g., Kippenhahn \& Weigert 1990)

$$
\frac{\partial r}{\partial m}=\frac{1}{4 \pi r^{2} \rho}
$$

Momentum conservation requires that the acceleration $\frac{\partial^{2} r}{\partial t^{2}}$ of a mass shell is composed of the gravitational acceleration $-\frac{G m}{r^{2}}$ ( $G$ is the gravitational constant) and the acceleration $4 \pi r^{2} \frac{\partial p}{\partial m}$ caused by the pressure gradient. It may thus be written as

$$
\frac{\partial p}{\partial m}=-\frac{G m}{4 \pi r^{4}}-\frac{1}{4 \pi r^{2}} \frac{\partial^{2} r}{\partial t^{2}}
$$

In the case of a hydrostatic configuration, the acceleration caused by the pressure gradient balances the gravitational acceleration and we have $\frac{\partial^{2} r}{\partial t^{2}}=0$.

\subsubsection{Energy conservation}

The luminosity $L$ passing through a spherical mass shell of a star may be affected by the following heat sinks and sources: Firstly, if nuclear reactions are taking place inside the shell, the nuclear energy generated and any energy losses caused by the escape of neutrinos have to be taken into account. Secondly, the heat content of the shell may 
change with time. The change of the luminosity across the mass shell is therefore given by

$$
\frac{\partial L}{\partial m}=\epsilon-\frac{\partial q}{\partial t}
$$

where $\epsilon$ is the specific nuclear energy generation rate, including possible energy losses by neutrinos, and $q$ is the specific heat content. According to the first law of thermodynamics, the change of the heat content of a mass element is composed of a change of its internal energy $d E$ and the mechanical work $p d V$ done on its surroundings. Therefore, equation 2.3 may also be written as

$$
\frac{\partial L}{\partial m}=\epsilon-\frac{p}{\rho^{2}} \frac{\partial \rho}{\partial t}-\frac{\partial E}{\partial t}
$$

Alternatively, using the second law of thermodynamics $(d q=T d s$, where $s$ is the specific entropy) and introducing the definitions $\alpha=\left.\frac{d \log \rho}{d \log p}\right|_{T}$ and $\delta=-\left.\frac{d \log \rho}{d \log T}\right|_{p}$ the relation

$$
\frac{\partial L}{\partial m}=\epsilon-c_{p} \frac{\partial T}{\partial t}+\frac{\delta}{\rho} \frac{\partial p}{\partial t}
$$

can be derived (see, e.g., Kippenhahn \& Weigert 1990). $c_{p}$ is the specific heat at constant pressure. Depending on the context, different formulations of energy conservation may be appropriate.

\subsubsection{Energy transport}

For the stellar models considered in this study, energy is transported by convection and radiation. The luminosity $L$ is therefore composed of a convective $\left(L_{\text {con }}\right)$ and a radiative ( $\left.L_{\mathrm{rad}}\right)$ part. In the interior of a star, energy transport by radiation can be described by a diffusion equation, i.e., the radiative luminosity is proportional to the mean free path of the photons and the gradient of the energy density. Expressing the gradient of the energy density in terms of the temperature gradient we obtain

$$
\frac{\partial T}{\partial m}=-\frac{G m T}{4 \pi r^{4} p} \nabla
$$

with

$$
\nabla=\nabla_{\mathrm{rad}}=\frac{3}{16 \pi a c G} \frac{\kappa p L_{\mathrm{rad}}}{m T^{4}}
$$

where $a$ is the radiation constant, $c$ the speed of light and $\kappa$ the Rosseland mean of the opacity of the stellar matter (see section 2.1.5).

Convection sets in when the temperature gradient becomes too steep. According to the Schwarzschild criterion for the onset of convection, this is the case if

$$
\frac{d \log T}{d \log p} \geq\left.\frac{d \log T}{d \log p}\right|_{\text {ad }}
$$

Then $\nabla$ in equation 2.6 is equal to $\nabla_{\text {mlt }}$, which is determined here by use of the mixing length theory (Böhm-Vitense 1958). 


\subsubsection{Equation of state}

To close the system of equations a relation between pressure $p$, density $\rho$ and temperature $T$ is needed. This is determined by the properties of the stellar matter. For the models considered, the stellar matter can be described as a mixture of ideal gas and radiation. Pressure $p$ and internal energy $E$ are then given by

$$
\begin{aligned}
p & =\frac{R}{\mu} \rho T+\frac{1}{3} a T^{4} \\
E & =\frac{3}{2} \frac{R}{\mu} T+\frac{a T^{4}}{\rho}+E_{\text {ion }}
\end{aligned}
$$

$R$ is the gas constant and $\mu$ the mean molecular weight, which depends on ionisation. $E_{\text {ion }}$ contains the contribution of ionisation to the internal energy. It has to be calculated from statistical equilibrium of the different ionisation states using the Saha equation. Ionisation energies are provided by atomic physics (see, e.g., Kippenhahn \& Weigert 1990).

\subsubsection{Opacity}

The Rosseland mean of the opacity is calculated from the frequency dependent opacity coefficient $\kappa_{\nu}$ by averaging with the frequency dependent flux $F_{\nu}$ :

$$
\kappa=\frac{\int_{0}^{\infty} \kappa_{\nu} F_{\nu} d \nu}{\int_{0}^{\infty} F_{\nu} d \nu}
$$

For large optical depths the flux $F_{\nu}$ is determined by the Planck function $B_{\nu}(T)$, and we get

$$
\frac{1}{\kappa}=\frac{\pi}{a c T^{3}} \int_{0}^{\infty} \frac{1}{\kappa_{\nu}} \frac{\partial B_{\nu}(T)}{\partial T} d \nu
$$

The opacity coefficient $\kappa_{\nu}$ has to be determined by atomic physics. In this study, the OPAL tables (Iglesias, Rogers \& Wilson 1992, Rogers \& Iglesias 1992) have been used for the Rosseland mean of the opacity.

\subsection{Stellar envelope models}

In the present study, the evolution of strange mode instabilities in the non-linear regime is examined. Since the stellar core remains largely unaffected by this type of instability, it is sufficient to restrict the computations to the stellar envelope, replacing the stellar core by suitable boundary conditions. Stellar envelopes can be constructed for given luminosity $L$, effective temperature $T_{\text {eff }}$ mass $M$ and chemical composition $(X, Y, Z)$, i.e., the observed chemical composition and position in the HR diagram together with an estimate for the mass. 
In the case of a hydrostatic stellar envelope in thermal equilibrium, equations 2.1, 2.2, 2.4 and 2.6 are considerably simplified. All time derivatives and the nuclear energy generation vanish. Therefore, the luminosity is constant in the envelope, and the problem is reduced to a third order ordinary differential system:

$$
\begin{aligned}
\frac{\partial r}{\partial m} & =\frac{1}{4 \pi r^{2} \rho} \\
\frac{\partial p}{\partial m} & =-\frac{G m}{4 \pi r^{4}} \\
\frac{\partial T}{\partial m} & =-\frac{G m T}{4 \pi r^{4} p} \nabla
\end{aligned}
$$

$\nabla$ is determined according to the mixing length theory. To integrate equations 2.132.15 , three boundary conditions are needed. One of them is given by the effective temperature $T_{\text {eff }}$ at $m=M$. Using the Stefan Boltzmann law

$$
L=4 \pi R^{2} \sigma_{S B} T_{\mathrm{eff}}^{4}
$$

the radius $R$ of the photosphere is determined by the given luminosity and effective temperature. (The Stefan Boltzman constant $\sigma_{S B}$ is equal to $\frac{a c}{4}$.) The third boundary condition is provided by the photospheric pressure $p_{\text {eff. }}$. It can be estimated by integrating the equation of hydrostatic equilibrium 2.13 and the definition of the optical depth $\tau$, $d \tau=-\kappa \rho d r$, from infinity to the photosphere. Denoting the opacity at the photosphere by $\kappa_{\text {eff }}$

$$
p_{\text {eff }}=\frac{2}{3} \frac{G M}{R^{2}} \frac{1}{\kappa_{\text {eff }}}
$$

is obtained. With these boundary conditions the differential system $2.13-2.15$ poses an initial value problem which is integrated from the photosphere to some maximum temperature $T_{\max }$. The numerical integration can be carried out by standard methods. We use a Runge-Kutta predictor corrector scheme which is accurate to fourth order (see, e.g., Press et al. 1992). 


\section{Linear stability analysis}

\subsection{Radial perturbation equations}

We consider the stability of hydrostatic envelope models with respect to infinitesimal radial perturbations. Let the hydrostatic background model be given by the functions $r_{0}(m), p_{0}(m), T_{0}(m)$ and $L_{0}(m)$ and the corresponding perturbations be denoted by $r^{\prime}(m), p^{\prime}(m), T^{\prime}(m)$ and $L^{\prime}(m)$ such that

$$
x(m, t)=x_{0}(m)+x^{\prime}(m, t) \quad \text { for } \quad \mathrm{x} \in\{\mathrm{r}, \mathrm{p}, \mathrm{T}, \mathrm{L}\}
$$

Assuming the perturbations to be infinitesimal, equations 2.1, 2.2,2.5 and 2.6 together with the equation of state and the opacity yield to first approximation a system of linear differential equations for the perturbations. Since the background model is time independent, the separation ansatz

$$
x^{\prime}(m, t)=x_{1}(m) \cdot e^{i \omega t} \quad \text { for } \quad \mathrm{x} \in\{\mathrm{r}, \mathrm{p}, \mathrm{T}, \mathrm{L}\}
$$

represents a solution and yields a system of ordinary differential equations with independent variable $m$ and $\omega$ as a parameter. Together with suitable boundary conditions we obtain a boundary eigenvalue problem for the eigenfrequency $\omega$. In general, $\omega$ will be complex, i.e., $\omega=\omega_{R}+i \omega_{I}$. Depending on the sign of $\omega_{I}$, perturbations will grow or decay exponentially. Negative values of $\omega_{I}$ indicate instability, positive values correspond to damped oscillations.

A form of the linear perturbation equations, which is particularly suitable for a numerical treatment was given by Baker and Kippenhahn (1962). Apart from a slight modification which avoids singularities in the coefficients (Gautschy \& Glatzel 1990a) we adopt essentially their notation. Convection is treated in the standard frozen in approximation (see Baker \& Kippenhahn 1965), i.e., the convective flux is kept constant and equal to its value in the hydrostatic envelope. The complete set of equations, including terms arising from nuclear energy generation which vanish for the envelope models considered here, then reads

$$
\begin{aligned}
& x^{2} \zeta^{\prime}=C_{4}^{*}\left(3 \zeta+C_{5} p-C_{6} t\right) \\
& x^{2} l^{\prime}=C_{1}^{*}\left(C_{10}^{*} \frac{d L_{0}}{d m} l-\left[(i \sigma)+C_{10} C_{12}\right] p+\left[(i \sigma) C_{2}-C_{10} C_{11}\right] t\right) \\
& p^{\prime}=-\left(4+C_{3} \sigma^{2}\right) \zeta-p \\
& t^{\prime}=C_{7}\left(-4 \zeta+C_{13} l+C_{8} p-C_{9} t\right)
\end{aligned}
$$


$\zeta, l, p$ and $t$ are the relative perturbations of radius, luminosity, pressure and temperature, respectively. The independent variable is $\ln p_{0}$. Correspondingly, dashes denote derivatives with respect to $\ln p_{0}$, i.e. $[\ldots]^{\prime}=\frac{d}{d \ln p_{0}}[\ldots] . \sigma=\omega \sqrt{R^{3} / 3 G M}$ is the eigenfrequency normalised to the global free fall time $\tau_{f f}$ and $x=r / R$ is the relative radius.

The coefficients in equations 3.3-3.6 are given by

$$
\begin{aligned}
C_{1} & =\sqrt{\frac{4 \pi \bar{\rho}}{G} \frac{4 \pi r^{4} P^{2} \delta}{m L \rho},} & C_{2} & =\frac{c_{p} \rho T}{P \delta} \\
C_{3} & =\frac{4 \pi r^{3}}{m} \bar{\rho}, & C_{4} & =\frac{P r}{G m \rho} \\
C_{5} & =\alpha, & C_{6} & =\delta \\
C_{7} & =\nabla_{0}, & C_{8} & =\left(\frac{\partial \log \kappa}{\partial \log P}\right)_{T} \\
C_{9} & =4-\left(\frac{\partial \log \kappa}{\partial \log T}\right)_{P}, & C_{10} & =\frac{\epsilon}{L} \frac{4 \pi r^{4} P}{G m C_{1}} \\
C_{10}^{*} & =\frac{4 \pi r^{4} P}{L G m C_{1}}, & C_{11} & =\left(\frac{\partial \log \epsilon}{\partial \log P}\right)_{T} \\
C_{12} & =\left(\frac{\partial \log \epsilon}{\partial \log T}\right)_{P}, & C_{13} & =\frac{L}{L^{R}}
\end{aligned}
$$

where $c_{p}$ is the specific heat at constant pressure, $L^{R}$ the radiative luminosity and $\bar{\rho}=$ $\frac{3 M}{4 \pi R^{3}}$ the mean density of the star. All quantities in equations 3.7 refer to the background model. To avoid singularities in the coefficients $C_{1,4}^{*}=x^{2} C_{1,4}$ are used instead of $C_{1}$ and $C_{4}$. The latter diverge as $r^{-2}$ for $r \rightarrow 0$. For envelope models $C_{10}, C_{11}$ and $C_{12}$ vanish.

\subsubsection{Boundary conditions}

Together with two inner and two outer boundary conditions the system of the linear ordinary differential equations 3.3-3.6 poses a boundary eigenvalue problem. Two of the boundary conditions are obtained from the condition that the solution has to be regular at $x=0$ :

$$
\begin{array}{cc}
3 \zeta+C_{5} p-c_{6} t & =0 \\
c_{10}^{*} \frac{d L_{0}}{d m} l-\left(i \sigma+C_{10} C_{11}\right) p+\left(i \sigma C_{2}-C_{10} C_{12}\right) t & =0
\end{array}
$$

The choice of the outer boundary conditions is ambiguous, since the boundary of the model does not coincide with the physical boundary of the star. For the thermal outer boundary condition we require the Stefan Boltzmann law to be valid, which in its linear form reads

$$
2 \zeta-l+4 t=0
$$


For the mechanical outer boundary condition we consider three possibilities:

$$
\begin{array}{cc}
p & =0 \\
\left(4+3 \sigma^{2}\right) \zeta+p & =0 \\
\alpha p-\delta t & =0
\end{array}
$$

The first condition corresponds to a force free boundary. The second condition is appropriate for an isothermal atmosphere and was derived by Baker and Kippenhahn (1965). They showed, that in this case the gradient of the relative pressure perturbation has to vanish at the outer boundary. Finally, the third condition implies a vanishing Lagrangian density perturbation.

Even if the ambiguity in the mechanical outer boundary condition seems unsatisfactory, it turns out to have negligible influence on the results of the linear stability analysis. This is due a strong gradient of the sound speed just below the photosphere, which leads to an almost complete reflection of sound waves, thus reducing the influence of the outer boundary condition.

\subsubsection{Approximations}

The solution of the perturbation equations 3.3-3.6 (see section 3.4) yields a spectrum of eigenfrequencies $\sigma$ and the corresponding eigenfunctions with the relative perturbations of radius (relative Lagrangian displacement), pressure, temperature and luminosity as components. Both modes and stability properties may have various physical origins. To distinguish between different types of modes and different physical processes several approximations to equations 3.3-3.6 may be considered. To obtain a continuous transition from the exact treatment to the approximation, it is convenient to introduce a parameter $\Phi$ with $\Phi=1$ corresponding to the exact problem and $\Phi \rightarrow 0, \infty$ to the approximation.

\section{Secular approximation}

Disregarding the restoring forces responsible for the existence of acoustic modes, the secular spectrum is isolated. This is achieved by introducing the parameter $\Phi$ into the Euler equation according to

$$
p^{\prime}=-\left(4+\Phi \cdot C_{3} \sigma^{2}\right) \zeta-p
$$

The limit $\Phi \rightarrow 0$ corresponds to vanishing acceleration. Should the considered mode still exist in the secular approximation is has to be of thermal origin. Vice versa, should the considered mode disappear in this limit, it has a mechanical origin.

Usually the acoustic and secular spectra are well separated, where the ratio of secular and acoustic eigenvalues corresponds to the ratio of Kelvin-Helmholtz and dynamical timescale. In the normalisation chosen this implies $|\sigma| \ll 1$ for the secular spectrum. 


\section{Adiabatic approximation}

The local dynamical timescale $\tau_{\text {dyn }}$ is defined as the time needed by a sound wave to travel across a shell of thickness $\Delta r$. The local thermal timescale $\tau_{\text {therm }}$ is the time needed to radiate the heat content of this shell at the local luminosity. Due to the dependence on $\Delta r$ only the ratio of these timescales is well defined:

$$
\frac{\tau_{\text {therm }}}{\tau_{\text {dyn }}}=\frac{4 \pi r^{2} \rho c_{p} T c_{s}}{L}
$$

The adiabatic approximation is valid if the timescale for the changes of state is small compared to that for heat exchange, i.e., if the ratio of thermal to dynamical timescale is large. From equation 3.12 we deduce that the deviations from adiabaticity increase with the luminosity to mass ratio.

In the adiabatic approximation an algebraic relation between relative temperature and pressure perturbations exists: $t=\nabla_{a d} \cdot p$. It decouples and closes the mechanical part of the perturbation equations (3.3 and 3.5), thus reducing the problem to a second order differential system. This may be written as a second order differential equation of the form

$$
\mathcal{L} \zeta=\sigma^{2} \zeta
$$

Together with the boundary conditions 3.8 and 3.10 the differential operator $\mathcal{L}$ is self adjoint. Thus equation 3.13 represents a standard Sturm-Liouville problem, which implies that all eigenvalues $\sigma^{2}$ are real. Therefore $\sigma$ is either real or purely imaginary corresponding to neutrally stable oscillations or non oscillatory instabilities. On the basis of a variational principle for equation 3.13 a sufficient condition for dynamical instability can be derived (see, e.g., Cox 1980): If the volumetric pressure weighted mean of the adiabatic index $\Gamma$ falls below $4 / 3$, the star is dynamically unstable.

From the exact problem the adiabatic approximation is obtained by introducing $\Phi$ into the equation for energy conservation according to

$$
l^{\prime}=C_{1} \cdot \Phi \cdot(i \sigma)\left(-p+C_{2} t\right)
$$

and taking the limit $\Phi \rightarrow \infty$.

\section{NAR approximation}

For stars characterised by large deviations from adiabatic behaviour the non-adiabatic reversible (NAR) or zero thermal timescale approximation provides an appropriate description. Writing the differential of the entropy as

$$
d s=c_{p} \frac{\alpha}{\delta}\left(\frac{d p}{p}-\Gamma_{1} \frac{d \rho}{\rho}\right)
$$

it is evident that the entropy can be kept constant by requiring either the term in brackets or the specific heat to vanish. The first case corresponds to the adiabatic, the second 
to the NAR approximation. Physically, the NAR-approximation implies that, due to a negligible specific heat of the envelope, luminosity perturbations cannot be sustained. Changes in the heat content of the stellar matter are radiated away instantly. Therefore, this approximation is valid if the ratio of thermal to dynamical timescale $\tau_{\text {therm }} / \tau_{\text {dyn }}$ is much smaller than unity. Should an instability exist in the NAR-limit the classical $\kappa$ mechanism is ruled out as its origin, since this Carnot-type process relies on a finite heat capacity.

Writing the coefficient $C_{1}$ in equation 3.7 as

$$
C_{1}=\frac{r_{0}^{2} p_{0}}{G m \rho_{0}} \sqrt{\frac{\rho_{0}}{\Gamma_{1} p_{0}}} \nabla_{\mathrm{ad}} \frac{\tau_{\text {therm }}}{\tau_{\mathrm{dyn}}}
$$

we see that the NAR-approximation is equivalent to the limit $C_{1} \rightarrow 0\left(\tau_{\text {therm }} / \tau_{\text {dyn }} \propto c_{p}\right)$. Therefore, it can be obtained from the exact problem by introducing $\Phi$ into the equation of energy conservation according to

$$
l^{\prime}=C_{1} \cdot \Phi \cdot(i \sigma)\left(-p+C_{2} t\right)
$$

and considering the limit $\Phi \rightarrow 0$. For $\Phi=0$ the solutions of the system 3.3-3.6 exhibit an additional symmetry: Since the term proportional to $i \sigma$ vanishes, the solutions occur in complex conjugate pairs, i.e., with $\sigma=\sigma_{R}+i \sigma_{I} \sigma^{*}=\sigma_{R}-i \sigma_{I}$ is a solution too. The physical meaning of this symmetry is the time reversibility of the system (hence the term non-adiabatic reversible approximation).

\subsection{The work integral}

The work integral is a widely used tool to identify which regions in the star drive or damp pulsations (see, e.g., Baker \& Kippenhahn 1962 or Cox 1980). For many cases it allows to identify the particular physical mechanisms responsible for instability. In general, it is interpreted as the amount of energy transferred within one pulsation period from thermal to mechanical energy by a Carnot-type process. This interpretation cannot hold for instabilities present in the NAR approximation, since Carnot processes rely on a finite specific heat. Furthermore, the work integral is derived for quasi periodic motions assuming a small ratio $\sigma_{I} / \sigma_{R}$. For the instabilities considered this ratio can be of order unity, thus violating the prerequisite of periodicity. In other words, a meaningful time average, necessary to define the work integral, does not exist.

To overcome this problem, Glatzel (1994) gave an alternative derivation of the work integral, replacing the ill defined time average by an ensemble average over equivalent states of the system. Considering a previously determined eigenfunction, the ambiguity in its phase factor is used to define the average (for a complete derivation, see appendix B). The result is the expression

$$
\frac{\sigma_{i}}{\sigma_{r}} \propto \int d V \frac{1}{\bar{\rho}} \mathfrak{I m}\left(\Delta p^{\dagger} \Delta \rho^{\dagger *}\right)
$$


for the work integral, which may be interpreted in the usual way $\left((\cdot)^{\dagger}\right.$ denotes the spatial part of the eigenfunction and $(\cdot)^{*}$ complex conjugation). The sign of the integrand in equation 3.18 determines whether a region in the star has a damping or exciting influence. The regions inducing instability, and with additional information also the mechanism responsible for it may thus be identified.

For an algebraic relation between (Lagrangian) pressure and density perturbation $\Delta p^{\dagger}=C \cdot \Delta \rho^{\dagger}$ with $C \in \mathbb{R}$ the integrand in equation 3.18 vanishes, implying stability $\left(\sigma_{I}=0\right)$. Physically, a phase shift between pressure and density perturbation is necessary for instability. The algebraic relation implies vanishing phase shift.

We note, that in general instability is attributed to the classical $\kappa$ mechanism, once driving is found close to an opacity maximum. However, as the work integral only indicates the position of driving, this conclusion has to be drawn with caution. For example, in ionisation zones opacity maxima and low values of $\beta$ are often found simultaneously ( $\beta$ is the ratio of gas pressure to total pressure). Thus instabilities depending on a low value of $\beta$ and $\kappa$-instabilities cannot be distinguished on the basis of the differential work integral.

\subsection{Mode interaction}

Considering the acoustic spectrum of stellar models as a function of stellar parameters such as mass or effective temperature, crossings of eigenfrequencies, i.e., mode resonances, are a common phenomenon. The basic properties of mode interactions will be discussed briefly in this section following the analysis by Cairns (1979) and Glatzel (1987).

Let us consider a system with two complex eigenfrequencies $\sigma_{1}$ and $\sigma_{2}$. If $\sigma_{1}$ and $\sigma_{2}$ are independent, they are given by separate dispersion relations:

$$
D_{1}\left(\sigma_{1}\right)=0 \text { and } \mathrm{D}_{2}\left(\sigma_{2}\right)=0
$$

If, however, a physical coupling between the modes exists, the two eigenfrequencies are the solutions of a single dispersion relation of the form

$$
D_{1}(\sigma) \cdot D_{2}(\sigma)=\epsilon(\sigma)
$$

where $\epsilon(\sigma)$ characterises the coupling of the modes. For weak coupling, i.e., for $\epsilon \ll$ $D_{1}, D_{2}$, the solution of equation 3.20 is approximately given by equation 3.19 . If, however, $D_{1}$ and $D_{2}$ have common roots, i.e., if the "independent" eigenfrequencies $\sigma_{1}$ and $\sigma_{2}$ cross each other, the condition $\epsilon \ll D_{1}, D_{2}$ cannot be satisfied. In this case, i.e., at the resonances of $\sigma_{1}$ and $\sigma_{2}$, the coupling between the modes has to be taken into account.

At a mode crossing $\sigma_{1}=\sigma_{2}=: \sigma_{c}$, where $\sigma_{1}$ and $\sigma_{2}$ are determined by equation 3.19 we introduce the difference $\delta$ of the uncoupled eigenvalues and the difference $\Delta$ of the correct eigenvalue $\sigma$ and the uncoupled eigenvalue $\sigma_{1}$ as new variables:

$$
\delta=\sigma_{2}-\sigma_{1} \quad \text { and } \quad \sigma=\sigma_{1}+\Delta
$$


Using equations 3.19 and 3.21 we expand $D_{1}$ and $D_{2}$ in terms of $\Delta$ and $\delta$ and obtain to first order in the approximation:

$$
\begin{aligned}
& D_{1}(\sigma)=\left.\Delta \frac{\partial D_{1}}{\partial \sigma}\right|_{\sigma_{c}} \\
& D_{2}(\sigma)=\left.(\Delta-\delta) \frac{\partial D_{2}}{\partial \sigma}\right|_{\sigma_{c}}
\end{aligned}
$$

Since $\epsilon$ is assumed to be small we may write $\epsilon(\sigma) \approx \epsilon\left(\sigma_{c}\right)$. Inserting this together with equation 3.22 into equation 3.20 a quadratic equation for $\Delta$ is obtained. Its solution is given by

$$
\Delta=\frac{\sigma_{2}-\sigma_{1}}{2} \pm\left[\frac{\left(\sigma_{2}-\sigma_{1}\right)^{2}}{4}+\frac{\epsilon\left(\sigma_{c}\right)}{\left.\left.\frac{\partial D_{1}}{\partial \sigma}\right|_{\sigma_{c}} \cdot \frac{\partial D_{2}}{\partial \sigma}\right|_{\sigma_{c}}}\right]^{\frac{1}{2}}
$$

Sufficiently far for the resonance (i.e., if the first term in brackets is much larger than the second one) the coupling term containing $\epsilon$ may be neglected and we get the uncoupled eigenvalues $\sigma=\sigma_{1}$ and $\sigma=\sigma_{2}$ as a solution. Near the crossing point we have $\sigma_{1} \approx \sigma_{2}$ and the solution is given by

$$
\Delta= \pm\left[\frac{\epsilon\left(\sigma_{c}\right)}{\left.\left.\frac{\partial D_{1}}{\partial \sigma}\right|_{\sigma_{c}} \cdot \frac{\partial D_{2}}{\partial \sigma}\right|_{\sigma_{c}}}\right]^{\frac{1}{2}}
$$

Let us now consider the case where $D_{1}, D_{2}$ and $\epsilon$ are real functions. Then the sign of the term under the square root of equation 3.24 determines whether we get a pair of complex conjugate eigenvalues or a pair of non-crossing real eigenvalues (provided $\sigma_{1}$ and $\sigma_{2}$ are real). The first case, where the eigenvalues $\sigma$ are given by a complex conjugate pair $\sigma=\sigma_{1} \pm i|\Delta|$, is usually addressed as "instability band". The second case, where the real eigenfrequencies avoid each other $\sigma=\sigma_{1} \pm|\Delta|$, is addressed as "avoided crossing". Qualitatively, these coupling schemes are still valid if $\sigma_{1}$ and $\sigma_{2}$ are complex. The modification then consists of a superposition of the coupling effect and the intrinsic imaginary parts of $\sigma_{1}$ and $\sigma_{2}$. Coupling via avoided crossing and instability band is illustrated in Figure 3.1.

\subsubsection{Stability of massive main-sequence stars}

Mode coupling is found in a stability analysis of massive main sequence stars (Glatzel \& Kiriakidis 1993a). These authors considered a zero age main sequence for the chemical composition $X=0.7, Y=0.27, Z=0.03$ with masses between $M=40 M_{\odot}$ and $M=120 M_{\odot}$. The linear stability analysis was performed following the frequencies of the lowest order modes as a function of the mass of the star, i.e., with respect to the previous section, the mass corresponds to the physical parameter $\lambda$ in Figure 3.1.

Figure 3.2 shows the real $\left(\sigma_{r}\right)$ and imaginary $\left(\sigma_{i}\right)$ parts of the lowest order radial eigenfrequencies as a function of the mass of the models. Following the frequencies 


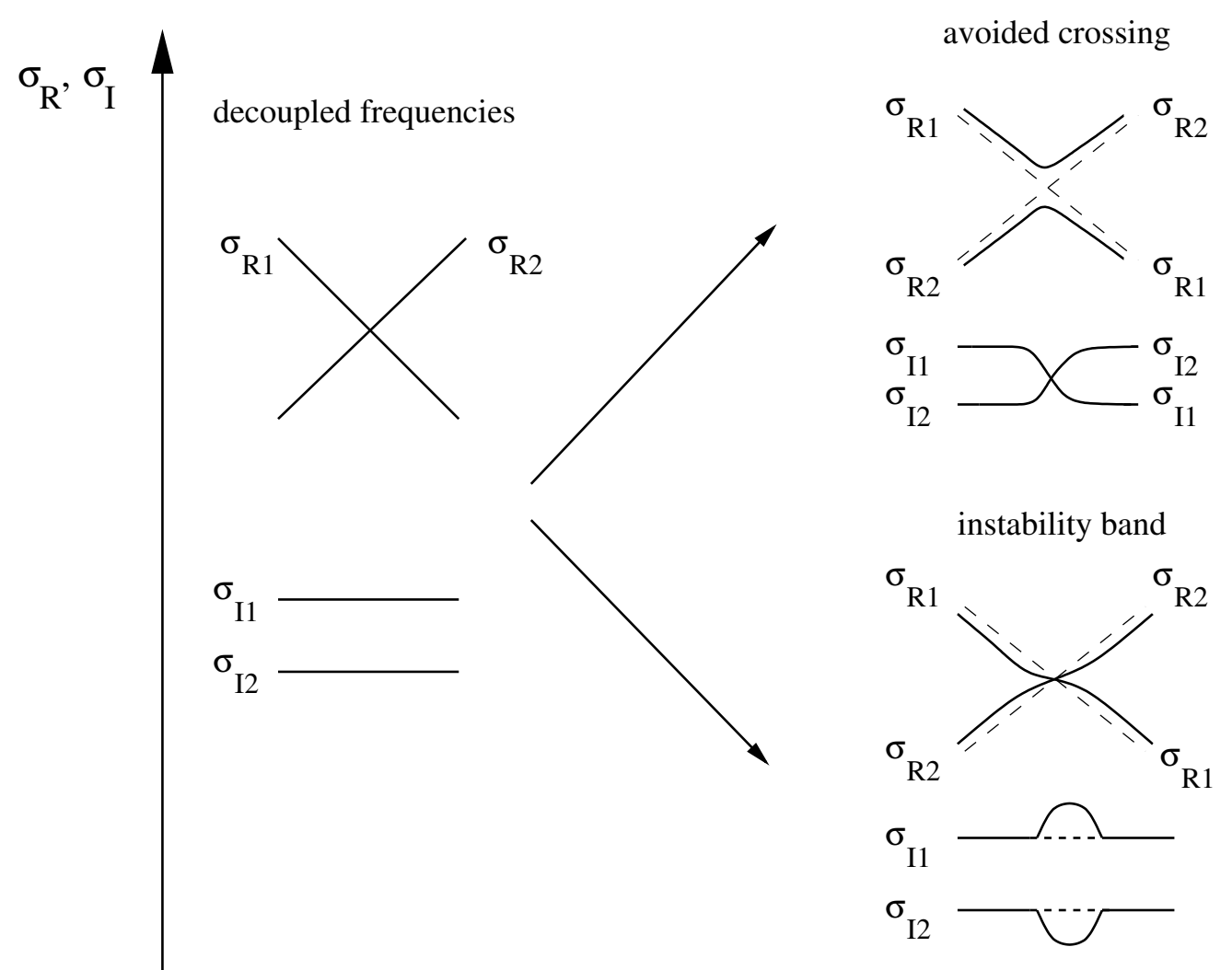

$\lambda$

Figure 3.1: Coupling schemes for the resonance of two modes. Real and imaginary parts of the eigenfrequencies $\sigma_{1}$ and $\sigma_{2}$ are given as a function of some parameter $\lambda$. The decoupled frequencies (left hand side) may couple to form an avoided crossing or an instability band (right hand side).

from low to high masses, a bewildering complexity of the modal diagram is encountered, including various mode crossings.

In order to classify the different modes, their counterparts in the NAR approximation have been calculated. The result is shown in Figure 3.3, where eigenfrequencies are neutrally stable or occur in complex conjugate pairs, according to the discussion presented in section 3.1.2. This facilitates the mode classification considerably. From Figure 3.3 we deduce, that two sets of modes are necessary to describe the modal diagram, one of which, consisting of neutrally stable modes with almost constant frequency, may be identified with the ordinary acoustic spectrum. The second set, consisting of complex conjugate mode pairs (labelled by $S n$ ) is formed at frequencies above $\sigma_{r} \approx 6$ by merging and coupling of neutral modes, corresponding to mode coupling by instability bands. Having very high imaginary parts, the real parts of the eigenfrequencies decrease with mass, thus crossing the ordinary acoustic spectrum.

By gradually switching on the irreversible term in the energy equation the frequen- 

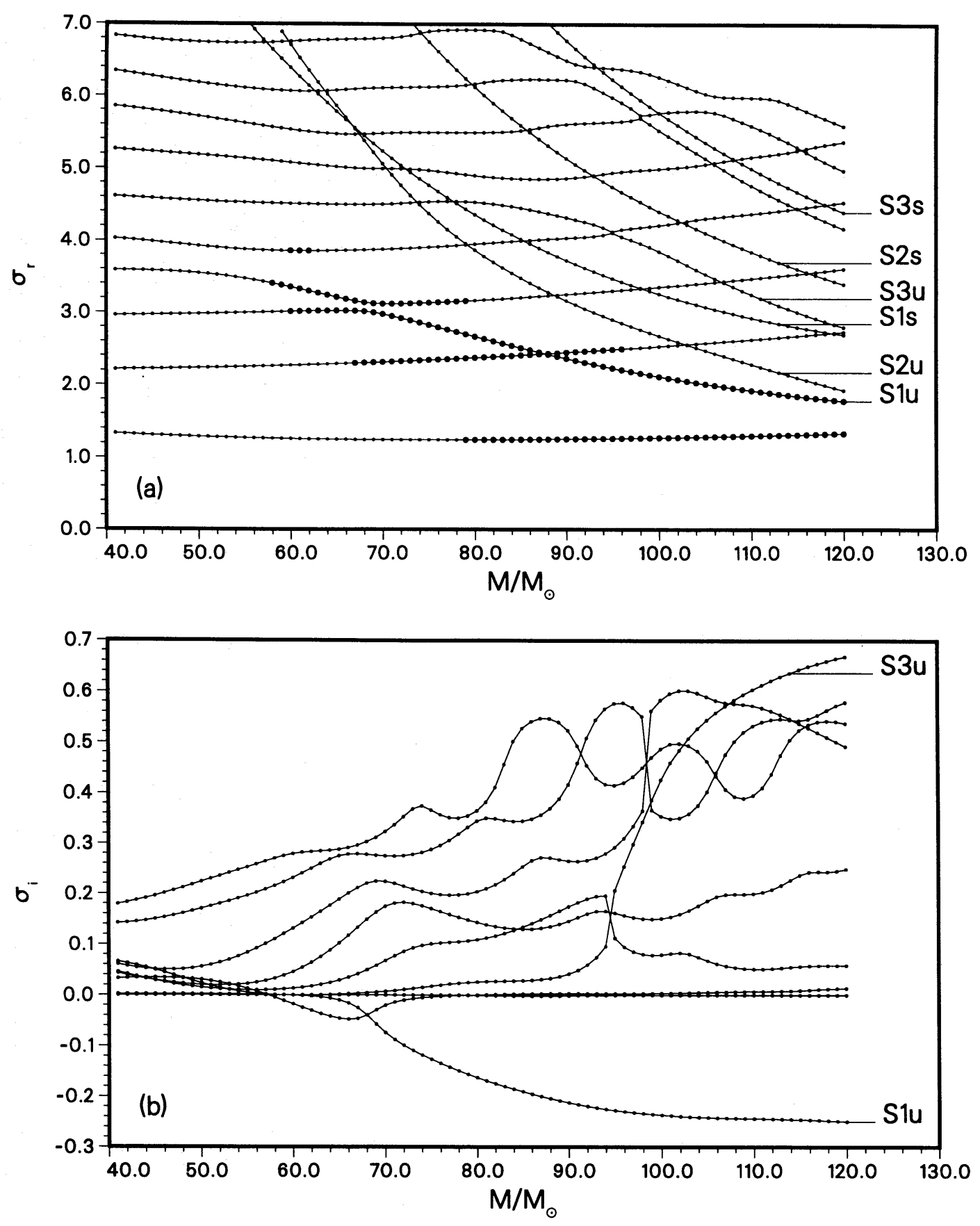

Figure 3.2: Real $\left(\sigma_{r}\right)$ and imaginary $\left(\sigma_{i}\right)$ parts of the eigenfrequencies normalised by the global free fall time of the lowest order radial modes as a function of mass for ZAMS models. In (a) large dots indicate unstable modes. This figure is taken from Glatzel \& Kiriakidis, 1993a. 

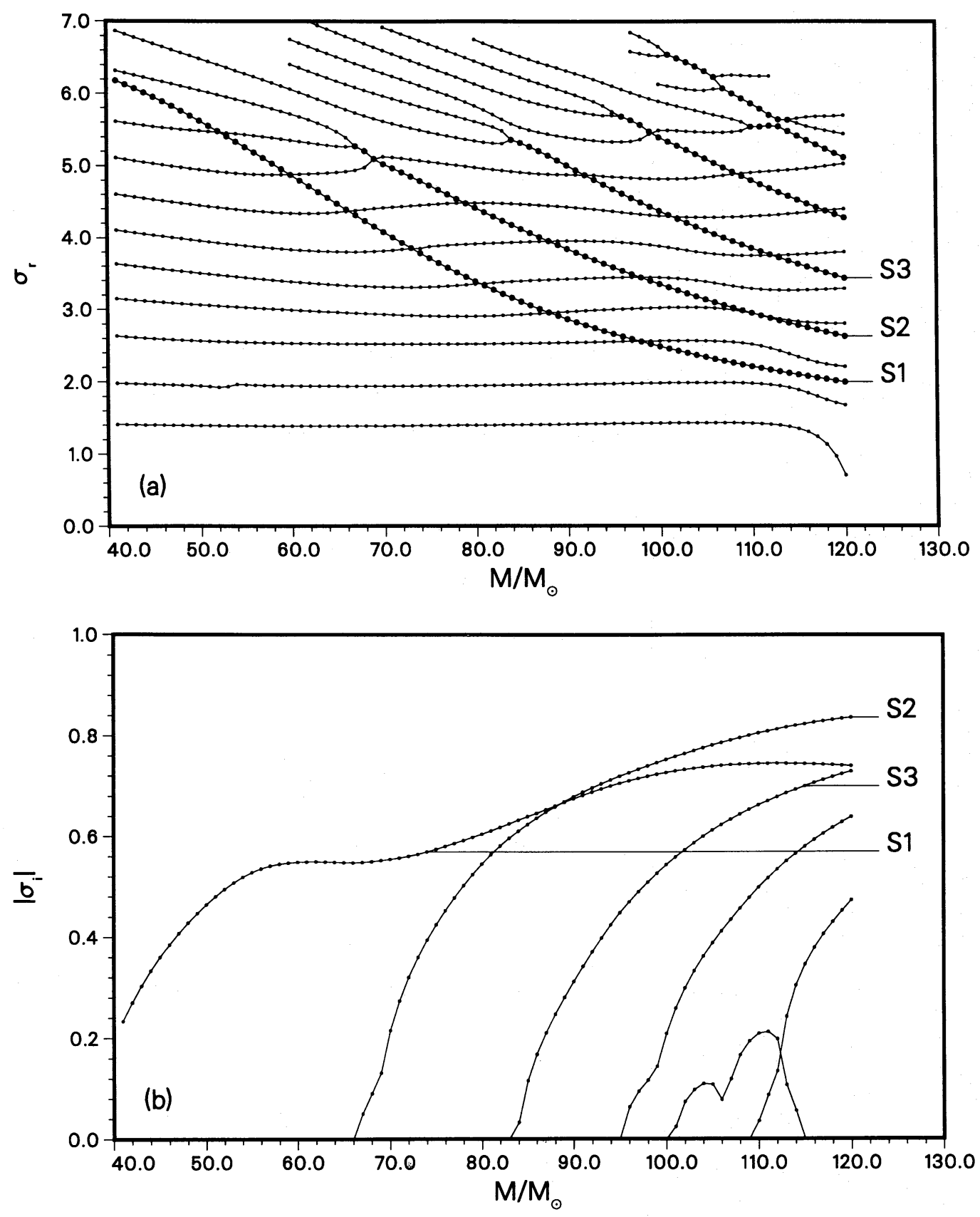

Figure 3.3: Same as Figure 3.2, but according to the NAR approximation. Note that eigenvalues occur in complex conjugate pairs. This figure is taken from Glatzel \& Kiriakidis, 1993a.

cies of the $S n$ modes could be followed continuously to their counterparts in the exact problem, thus enabling a classification of modes in Figure 3.2. During this process, the symmetry of the eigenvalues is lost, i.e., the complex conjugate unstable ( $\mathrm{Snu}$ ) and 
damped (Sns) NAR branches do not show any symmetry or relation in the exact problem. Except for the $S 1 u$ branch, which is unstable for masses above $58 M_{\odot}$ all $S$ modes become stable in the exact treatment. Within the first three $S n$ pairs only $S 1 u$ and $S 3 u$ show interactions with the ordinary acoustic spectrum, where crossings with ordinary modes above the first (with $S 1 u$ ) or third (with $S 3 u$ ) overtone have unfolded into avoided crossings.

This discussion demonstrates that the NAR approximation is useful when interpreting modal diagrams, since then the mode coupling phenomena are obtained in their pure form. For the models considered by Glatzel and Kiriakidis (1993a), the NAR approximation yields only qualitatively correct results and eigenfrequencies can differ significantly in the exact treatment and the NAR approximation. For other objects, such as WolfRayet stars, the NAR treatment yields even quantitatively correct results (see Glatzel, Kiriakidis \& Fricke 1993 and Kiriakidis, Glatzel \& Fricke 1996).

\subsubsection{Physical interpretation}

Mode coupling phenomena are well known in many branches of physics as, e.g., hydrodynamics (Hansen et al. 1976, Glatzel 1987) and plasma physics (Chen 1987). There, the occurrence of avoided crossings and instability bands is sometimes explained in terms of the "pseudo energy" of the interacting modes: Avoided crossings occur if two modes cross whose energy has the same sign. If the sign differs instability bands are generated. In this case growing amplitudes may be interpreted by an exchange of energy among the modes, while the total energy of the system is kept constant. However, in the case of stellar pulsations, no suitable definition for the mode energy has been found so far.

In the context of stellar oscillations, resonances between modes were first discussed by Osaki (1975) who investigated the non-radial adiabatic pulsations of a $10 M_{\odot}$ star. Since then, also instabilities associated with mode coupling phenomena have been identified in a variety of stars both for radial and non-radial perturbations. These include extreme helium stars (Glatzel \& Gautschy1992), massive stars (Glatzel \& Kiriakidis 1993a, Glatzel \& Kiriakidis 1993b, Kiriakidis, Fricke \& Glatzel 1993, non-radial: Glatzel \& Mehren 1996), Wolf-Rayet stars (Glatzel, Kiriakidis \& Fricke 1993, non-radial: Glatzel \& Kaltschmidt 2002), Luminous Blue Variables (Kiriakidis 1992) and the central stars of planetary nebulae (Gautschy 1993). In all cases large deviations from adiabatic behaviour appear to be important, i.e., for instability the ratio of thermal to dynamical timescale has to be small for a large fraction of the stellar envelope. In other words, the ratio of luminosity to mass has to be large ( $>10^{4}$ in solar units). All models with these properties exhibit a pronounced core-envelope structure.

Mode coupling may provide the impression of additional modes (see section 3.3.1). Their unexpected appearance gave rise to the term "strange modes". Concerning their physical origin Gautschy \& Glatzel (1992) argued that strange modes are of acoustic rather than of thermal origin (Shibahashi \& Osaki 1981; Zalewski 1992). A mechanical origin is supported by the fact that the frequencies of strange modes are related to the 
sound travel time between the stellar surface and the position of the outermost opacity peak (Glatzel \& Kiriakidis 1993b). Finally, the instabilities associated with strange modes do exist in the NAR approximation. This proves their mechanical origin, since in this approximation thermal modes as well as the classical instability mechanisms ( $\kappa$ and $\epsilon)$ are excluded.

In a subsequent publication, Glatzel (1994) considered the perturbation problem for vanishing luminosity perturbations (NAR-approximation) and in the limit of negligible gas pressure $(\beta \ll 1)$. He showed, that under these conditions the pressure perturbation is proportional to the density perturbation for high and low wavenumbers. For low wave numbers the sound velocity defined by this proportionality is given by

$$
c_{\kappa}^{2}=\left.\frac{d p}{d \rho}\right|_{\kappa}=\frac{4 \kappa_{\rho}}{-\kappa_{T}} \frac{p_{0}}{\rho_{0}}
$$

where $\kappa_{\rho}$ and $\kappa_{T}$ denote the logarithmic derivatives of the opacity with respect to density and temperature, respectively. It becomes imaginary for positive values of $\kappa_{T}$, which corresponds to evanescent acoustic waves. As a consequence, regions having $\kappa_{T}>0$ act as acoustic barriers dividing the star into several acoustic cavities, each of them providing a separate acoustic spectrum. Each opacity peak (at $\log T \approx 5.3,4.7,4.0$, due to heavy elements, HeII and HeI ionisation, respectively) is associated with a region having $\kappa_{T}>$ 0 , and therefore with an acoustic barrier whose position may change during the evolution of the star. The acoustic spectrum may therefore be understood as a superposition of different sets of modes belonging to the various acoustic cavities and resonances between them.

With respect to a mechanism of the instabilities Glatzel (1994) demonstrated that a phase shift between pressure and density perturbation is a necessary condition for instability (see section 3.2). For high and low wavenumbers the proportionality between pressure and density perturbation implies a vanishing phase lag and thus only allows for neutrally stable oscillatory modes. However, in an intermediate range of wave numbers whose width depends on $\beta$ pressure and density perturbation are connected by a differential relation, which provides a phase shift between them and thus produces damped and excited solutions in complex conjugate pairs (NAR-approximation). According to this study in addition to short thermal timescales dominant radiation pressure is essential for the instability to exist. These conditions prevail in the extended envelopes of hot and luminous objects.

\subsection{Numerical method}

To solve the perturbation equations 3.3-3.6 together with the boundary conditions 3.8, 3.9 and 3.10 we use a method proposed by Gautschy and Glatzel (1990b). It is based on the Riccati method introduced by Scott (1973) and advanced by Davey (1977). As a first 
step the four dependent variables are redistributed to two complex vectors:

$$
\mathbf{u}=\left(\begin{array}{l}
\zeta \\
l
\end{array}\right) \quad \text { and } \quad \mathbf{v}=\left(\begin{array}{l}
t \\
p
\end{array}\right)
$$

The system 3.3-3.6 can then be written in the form

$$
\begin{aligned}
\Lambda \mathbf{u}^{\prime} & =\mathbf{A u}+\mathbf{B} \mathbf{v} \\
\mathbf{v}^{\prime} & =\mathbf{C u}+\mathbf{D} \mathbf{v}
\end{aligned}
$$

with $\Lambda=\operatorname{diag}\left(\mathrm{x}^{2}, \mathrm{x}^{2}\right)$ and $2 \times 2$ matrices $\mathbf{A}, \mathbf{B}, \mathbf{C}$ and $\mathbf{D}$, which contain the background coefficients of equations 3.3-3.6. Introducing the $2 \times 2$ Riccati matrix $\mathbf{R}$ and its inverse $\mathbf{S}=\mathbf{R}^{-1}$ by

$$
\begin{aligned}
& \mathbf{u}=\mathbf{R} \mathbf{v} \\
& \mathbf{v}=\mathbf{S u}
\end{aligned}
$$

a nonlinear matrix differential equation for $\mathbf{R}$ and $\mathbf{S}$, respectively, is obtained using equations 3.26:

$$
\begin{aligned}
\Lambda \mathbf{R}^{\prime} & =\mathbf{A} \mathbf{R}+\mathbf{B}-\Lambda \mathbf{R}(\mathbf{C R}+\mathbf{D}) \\
\Lambda \mathbf{S}^{\prime} & =\Lambda(\mathbf{C}+\mathbf{D S})-\mathbf{S}(\mathbf{A}+\mathbf{B S})
\end{aligned}
$$

These non linear matrix differential equations are the Riccati equations. In the standard form $\Lambda$ is the unit matrix. For given $\mathbf{R}$ or $\mathbf{S} \mathbf{v}$ or $\mathbf{u}$ satisfy a linear differential equation:

$$
\begin{aligned}
\mathbf{v}^{\prime} & =(\mathbf{C R}+\mathbf{D}) \mathbf{v} \\
\mathbf{u}^{\prime} & =\Lambda^{-1}(\mathbf{A}+\mathbf{B S}) \mathbf{u}
\end{aligned}
$$

Both the boundary conditions at the centre (3.8) and at the surface $(3.9,3.10)$ can be written in the form

$$
\mathbf{E u}=\mathbf{F v}
$$

where $\mathbf{E}$ and $\mathbf{F}$ are $2 \times 2$ matrices, which can be read off from equations 3.8-3.10. For the Riccati matrix $\mathbf{R}$ and its inverse $\mathbf{S}$ these conditions imply

$$
\mathbf{R}=\mathbf{E}^{-1} \mathbf{F} \quad \text { and } \quad \mathbf{S}=\mathbf{F}^{-1} \mathbf{E}
$$

Depending on which of the matrices $\mathbf{E}$ or $\mathbf{F}$ is singular at $x=0,1$ this yields unambiguous values for the Riccati matrix $\mathbf{R}$ or its inverse $\mathbf{S}$ there. Thus the boundary value problem has been transformed into an initial value problem. The matrices $\mathbf{R}$ or $\mathbf{S}$ can then be integrated from the boundaries $x=0,1$ towards some point $x_{f i t}$ within the integration interval. Requiring the variables $\mathbf{u}$ and $\mathbf{v}$ to be continuous at $x_{f i t}$ we have

$$
\begin{array}{ll}
\mathbf{u}^{\text {in }}\left(x_{f i t}\right) & =\mathbf{u}^{\text {out }}\left(x_{f i t}\right) \\
\mathbf{v}^{\text {in }}\left(x_{f i t}\right) & =\mathbf{v}^{\text {out }}\left(x_{f i t}\right)
\end{array}
$$


where the indices "in" and "out" denote the quantities determined by the integration from $x=0$ and $x=1$ to $x_{f i t}$, respectively. For the matrices $\mathbf{R}$ and $\mathbf{S}$ the continuity requirements imply

$$
\begin{array}{r}
\left(\mathbf{R}^{\text {in }}\left(x_{f i t}\right)-\left(\mathbf{R}^{\text {out }}\left(x_{f i t}\right)\right) \mathbf{v}=0\right. \\
\left(\mathbf{S}^{\text {in }}\left(x_{f i t}\right)-\left(\mathbf{S}^{\text {out }}\left(x_{f i t}\right)\right) \mathbf{u}=0\right.
\end{array}
$$

A necessary condition for equations 3.36 to have non-trivial solutions is

$$
\begin{array}{cc}
\operatorname{det}\left(\mathbf{R}^{\text {in }}\left(x_{f i t}\right)-\left(\mathbf{R}^{\text {out }}\left(x_{f i t}\right)\right)\right. & =0 \\
\operatorname{det}\left(\mathbf{S}^{\text {in }}\left(x_{f i t}\right)-\left(\mathbf{S}^{\text {out }}\left(x_{f i t}\right)\right)\right. & =0
\end{array}
$$

The only free parameter in these equations is the complex eigenfrequency $\sigma$. Therefore they represent the dispersion relation for $\sigma$ and the problem of determining the eigenfrequencies has thus been reduced to finding the (complex) root of a complex equation. The latter can be achieved iteratively by using, e.g., a complex secant method (see, e.g., Castor 1971). If $x_{f i t}$ corresponds to one of the boundaries the standard Riccati method described by Davey (1977) is recovered. Apart from singularities in $\mathbf{R}$ and $\mathbf{S}$, the Riccati procedure can may be applied equivalently either in terms of $\mathbf{R}$ or $\mathbf{S}$. To avoid singularities, the equations for $\mathbf{S}$ or $\mathbf{R}$ are used, if $|\operatorname{det} \mathbf{R}|$ or $|\operatorname{det} \mathbf{S}|$ exceeds unity, respectively.

One of the main advantages of the Riccati method is that initial guesses for the eigenvalues $\sigma$ can be obtained independently and without any previous knowledge by evaluating the determinant function 3.37. Another advantage is that the independent variable can easily be changed to achieve the optimal resolution for the integration. In the stellar centre the relative radius $x=r_{0} / R$ changes rapidly, whereas $\ln p_{0}$ varies quickly in the outer envelope. Therefore, $x$ and $\ln p_{0}$ are chosen as independent variables for the in-out and out-in integration, respectively.

Once the eigenvalues are known and the Riccati matrices $\mathbf{R}$ and $\mathbf{S}$ have been determined, equations 3.31 can be integrated to calculate the eigenfunctions. Starting with the values of $\mathbf{v}$ at $x_{f i t}$ as determined by equation 3.36 the integration is performed from the fit point towards the boundaries. $\mathbf{u}$ is then determined by $\mathbf{R}$ and $\mathbf{v}$ using $\mathbf{u}=\mathbf{R v}$. We emphasise that for numerical stability, $\mathbf{v}$ and $\mathbf{R}$ have to be integrated in opposite directions. Moreover, $\mathbf{R}$ and $\mathbf{v}$ must not be integrated simultaneously.

Compared to other shooting methods, the Riccati method has the advantage that at least one direction of integration is numerically stable, where the freedom in the choice of $x_{f i t}$ may be used to guarantee numerical stability. It turns out that the stability requirements still allow for a wide range of possible values for $x_{f i t}$. The remaining freedom is used to provide a functional behaviour of the determinant function which allows for fast and robust eigenvalue iteration. For optimum convergence it may be necessary to use different values of $x_{f i t}$ for different modes. 


\section{Non-linear evolution of instabilities}

To study the final results of strange mode instabilities in massive stars their evolution has to be followed into the non linear regime. After the linear phase of exponential growth the amplitudes saturate due to non-linear effects such as energy dissipation by shock waves. Whether strange mode instabilities ultimately lead to the loss of mass from the object depends on the amplitude reached. If velocity amplitude exceeds the escape velocity at the stellar surface mass is lost to the interstellar medium. Glatzel et al. (1999) have reported, that for particular models the amplitude has exceeded the escape velocity, but so far this has not been verified (Dorfi \& Gautschy 2000). If mass loss is found to be a periodic process, mean mass loss rates could directly be determined from non-linear simulations.

The instabilities investigated here have been identified not only for radial, but also for non-radial perturbations (Glatzel \& Mehren 1996; Glatzel \& Kaltschmidt 2002) with growth rates that are comparable to those found for radial instabilities. The results of purely radial calculations therefore have to be interpreted with some caution. Considering the spatial structure of the nebula surrounding LBVs, such as the Homunculus nebula surrounding Eta Carinae (Figure 1.3), non-radial calculations appear to be indicated.

The results of the non-linear simulation of the evolution of strange mode instabilities strongly depend on an appropriate numerical treatment. In particular, special attention has to be paid to the strict conservativity of the applied difference scheme, since the kinetic energy contained in the perturbation is typically smaller than the total energy of the system by several orders of magnitude. Even if the conservation of the total energy is approximately satisfied, violation of strict conservativity can therefore lead to considerable errors. This problem is overcome by the use of a fully conservative difference scheme, like that proposed by Fraley (1968) and Samarskii and Popov (1969). It relies on a Lagrangian description and time discretisation is fully implicit. A consistent extension of the code to two dimensions based on the method of support operators (Samarskii et. al. 1981; Ardelyan \& Gushchin 1982) is currently being developed (see Chernigovski, Glatzel, \& Fricke 2000).

\subsection{Relaxation of initial models}

The spatial distribution of gridpoints of an envelope integration as described in section 2.2 is in general not compatible with the numerical scheme used in the dynamical cal- 
culations. In particular, the number of gridpoints is too high. After the construction of the hydrostatic envelope model it is therefore adapted to a suitable grid with a reasonable number of gridpoints $(\sim 500-1000)$ but still properly resolving ionisation zones and instability regions.

For incompatible difference schemes, numerical errors induce perturbations with amplitudes in the non-linear regime. This problem may be overcome by damping the artificial perturbations using dissipation and viscosity or by a pseudo time evolution of the grid (Dorfi \& Drury 1987; Dorfi \& Feuchtinger 1995; Dorfi 1998). Using these techniques, however, the stellar models have to be perturbed externally to trigger the physical instability and envelope pulsations. Typically, initial velocity amplitudes of the order of $10 \mathrm{~km} \mathrm{sec}^{-1}$ amounting to 10 per cent of the local sound speed are then required (see, e.g., Dorfi \& Gautschy 2000).

In our approach the adaption of the hydrostatic envelope model to a suitable grid is achieved by reintegrating the envelope using the same difference scheme as that applied for the dynamical calculations and using the results of the standard envelope integration as initial guesses for the integration procedure. It implies, that - apart from the initial boundary values - only the convective flux is taken from the predetermined envelope model. Using this approach, hydrostatic equilibrium is satisfied to within round-off errors, rather than to $\mathcal{O}\left(\frac{1}{N}\right)$, which is the difference between incompatible difference schemes ( $N$ is the number of gridpoints). Thus, no artificial, numerically induced perturbations are present and no additional damping is required. Physical instabilities and stellar pulsations then develop without any further (unphysical) external perturbation from numerical noise, which (for the models considered here) corresponds to velocities of the order of a few $\mathrm{cm} \mathrm{sec}^{-1}$.

\subsection{Non-linear equations}

The evolution of instabilities of a stellar envelope is followed into the non-linear regime assuming spherical symmetry and adopting a Lagrangian description. Rotation and magnetic fields are neglected. The independent variables are the time $t$ and the (normalised) mass $s$ which is defined by $d s=\rho(r, 0) r^{2} d r$. For application of the Fraley scheme (Fraley 1968), the equations to be solved are written as

$$
\begin{aligned}
\frac{\partial}{\partial t}\left(\frac{1}{\rho}\right) & =\frac{\partial\left(r^{2} v\right)}{\partial s} \\
\frac{\partial v}{\partial t} & =-r^{2} \frac{\partial p}{\partial s}-\frac{4 \pi G M_{r}}{r^{2}} \\
\frac{\partial \epsilon}{\partial t} & =-p \frac{\partial\left(r^{2} v\right)}{\partial s}-\frac{\partial\left(r^{2}\left(F_{\mathrm{rad}}+F_{\mathrm{con}}\right)\right)}{\partial s} \\
F_{\mathrm{rad}} & =-\theta r^{2} \frac{\partial T}{\partial s}
\end{aligned}
$$


They correspond to mass conservation (4.1), momentum conservation (4.2), energy conservation (4.3) and the diffusion equation for energy transport (4.4). $\rho, p, T, r, v$ and $\epsilon$ denote density, pressure, temperature, radius, velocity and specific internal energy, re-

spectively, and $\frac{\partial}{\partial t}$ is the substantial time derivative. Apart from a factor $4 \pi M_{r}$ is the mass inside a sphere of radius $r, G$ is the gravitational constant and

$$
\theta=\frac{4 a c T^{3}}{3 \kappa \rho}
$$

is the diffusion coefficient, which contains the radiation constant $a$, the speed of light $c$ and the opacity $\kappa$. The latter is given by the OPAL tables (Iglesias, Rogers \& Wilson 1992; Rogers \& Iglesias 1992). $F_{\text {rad }}$ and $F_{\text {con }}$ are the radiative and the convective flux, respectively. The latter is kept constant during the evolution and equal to its initial value, in accordance with the standard frozen in approximation (see Baker \& Kippenhahn 1965). The system of equations is closed by the definition of the velocity $v=\frac{\partial r}{\partial t}$ and the equations of state $p=\mathcal{P}(\rho, T)$ and $\epsilon=\mathcal{E}(\rho, T)$.

Since the instabilities are localised in the outer envelope, the evolution of the core is neglected. Its properties are taken into account by imposing time independent boundary conditions (i.e., by prescribing luminosity, [vanishing] velocity $v$ and [constant] radius) at the bottom of the envelope. At the outer boundary, the gradient of heat sources is required to vanish:

$$
\operatorname{grad}(\operatorname{div} F)=0
$$

This boundary condition implies (by using equations 4.1,4.3 and 4.4) boundary values for the temperature $T$ and the pressure $p$. It is chosen to ensure that outgoing shocks pass the boundary without reflection.

\subsection{Numerical method}

The equations governing the evolution of instabilities in the stellar envelope (equations 4.1-4.4) are discretised on a staggered mesh such that the radius $r$, the velocity $v$, the radiative and convective flux $F_{\text {rad }}$ and $F_{\text {con }}$ and the mass inside a sphere of radius $r$ (including the mass of the core) $M_{r} / 4 \pi$ are defined on the nodes of the grid, whereas the density $\rho$, the pressure $p$, the specific internal energy $\epsilon$, the temperature $T$ and the diffusion coefficient $\theta$ are defined in the cells of the grid. Thus we obtain for the discretised form of equations 4.1-4.4

$$
\begin{aligned}
\left(\frac{1}{\hat{\rho}_{i}}-\frac{1}{\rho_{i}}\right) \cdot \frac{1}{\tau} & =\frac{\left(R_{i+1} \cdot v_{i+1}^{(0.5)}-R_{i} \cdot v_{i}^{(0.5)}\right)}{m_{i}} \\
\frac{\hat{v}_{i}-v_{i}}{\tau} & =-R_{i} \cdot \frac{\left(g_{i}^{(\alpha)}-g_{i-1}^{(\alpha)}\right)}{\bar{m}_{i}}-\frac{4 \pi G M_{r_{i}}}{\hat{r}_{i} r_{i}}
\end{aligned}
$$




$$
\begin{aligned}
\frac{\hat{\epsilon}_{i}-\epsilon_{i}}{\tau}= & -g_{i}^{(\alpha)} \cdot \frac{\left(R_{i+1} \cdot v_{i+1}^{(0.5)}-R_{i} \cdot v_{i}^{(0.5)}\right)}{m_{i}} \\
& -\frac{R_{i+1} \cdot\left(F_{\mathrm{rad}, \mathrm{i}+1}^{(\sigma)}+F_{\mathrm{con}, \mathrm{i}+1}\right)}{m_{i}} \\
& +\frac{R_{i} \cdot\left(F_{\mathrm{rad}, \mathrm{i}}^{(\sigma)}+F_{\mathrm{con}, \mathrm{i}}\right)}{m_{i}} \\
F_{\text {rad }, \mathrm{i}}= & -\bar{\theta}_{i} \cdot r_{i}^{2} \cdot \frac{T_{i}-T_{i-1}}{\bar{m}_{i}}
\end{aligned}
$$

$i$ denotes the number of the corresponding node/cell, with $i$ increasing outwards. $m_{i}=$ $\rho_{i}\left(r_{i}^{3}-r_{i-1}^{3}\right) / 3$ denotes the mass of cell $i$ and $\bar{m}_{i}=\frac{1}{2} \cdot\left(m_{i}+m_{i-1}\right)$ the mass associated with node $i$, respectively. $g$ is the sum of pressure $p$ and artificial viscosity (volume or tensor artificial viscosity).

$$
\bar{\theta}_{i}=\sqrt{\theta_{i-1}\left(p_{i-1}, T_{i-1}\right) \cdot \theta_{i}\left(p_{i}, T_{i}\right)}
$$

maps the diffusion coefficient to the nodes of the grid. $R_{i}$ is given by

$$
R_{i}=\frac{1}{3}\left(\hat{r}_{i}^{2}+\hat{r}_{i} r_{i}+r_{i}^{2}\right)
$$

The definition of the velocity

$$
\frac{\hat{r}_{i}-r_{i}}{\tau}=v_{i}^{(0.5)}
$$

and the equations of state

$$
\begin{aligned}
p_{i} & =\mathcal{P}\left(\rho_{i}, T_{i}\right) \\
\epsilon_{i} & =\mathcal{E}\left(\rho_{i}, T_{i}\right)
\end{aligned}
$$

close the system of equations.

The scheme is implicit with respect to time where quantities $\hat{x}$ and $x$ refer to the time $t_{n+1}$ and $t_{n}$, respectively. ( $t_{n+1}=t_{n}+\tau, \tau$ is the variable integration timestep.) $\alpha$ and $\sigma \in[0,1]$ are free weight parameters, and time averaging is done according to

$$
x^{(\alpha)}=\alpha \cdot \hat{x}+(1-\alpha) \cdot x
$$

In the presented simulations, the values $\alpha=\sigma=1$ have been adopted.

For time integration the system of equations 4.7-4.10 is split into the mechanical part, consisting of equations 4.7-4.8, and the energy part consisting of equations 4.9-4.10. Then time integration proceeds according to the following scheme:

1. Keeping $\tilde{T}$ and $\tilde{F}_{\text {rad }}$ fixed the mechanical part of the equations is advanced in time. This yields values for the velocity $\tilde{v}$ and the pressure $\tilde{p}$ at time $t_{n+1}$.

2. Keeping $\tilde{v}$ and $\tilde{p}$ fixed, the energy part of the equations is advanced in time. This yields values for the temperature $\tilde{T}$ and the radiative flux $\tilde{F}_{\text {rad }}$ at time $t_{n+1}$. 
3. Using $\tilde{v}, \tilde{p}, \tilde{T}$ and $\tilde{F}_{\text {rad }}$ as new guesses at the timelevel $t_{n+1}$ integration according to (1) and (2) is repeated iteratively until the full set of equations is satisfied to within prescribed accuracy.

Since we apply an implicit difference scheme, the time advancement of each of the steps (1) and (2) has to be done iteratively. The convergence of the procedure (1)-(3) can be considerably accelerated, if an explicit time integration is used for the first iteration step of (1) and (2). Then the scheme is still fully implicit since the values thus obtained are used only as initial guesses.

For each of the steps (1)-(3) an individual error bound may be defined. Convergence is controlled using the following criterion based on a $l^{2}$-norm:

$$
\frac{1}{N}\left(\sum_{i} f_{i}^{2}\right)^{\frac{1}{2}}<E_{x}
$$

where $f_{i}$ denotes the relative error of a physical quantity in gridpoint or cell $i$ and $N$ is the total number of gridpoints. $E_{x}$ is one of the prescribed error bounds $E_{\text {mech }}, E_{\text {energ }}$ and $E_{\text {tot }}$ (corresponding to the mechanical part, the energy part and the complete system of equations, respectively) and the sum extends over all gridpoints. $f_{i}^{2}$ contains the weight-function of the $l^{2}$-norm which is chosen to be proportional to the mass of the corresponding cell, $f_{i}^{2} \propto m_{i}$.

The timestep $\tau$ is adjusted to provide optimal convergence for the various iteration cycles described above. For this purpose, a range is specified for the number of iterations within the mechanical and energy parts and the complete system. If the number of iterations leaves this range, the timestep is reduced or increased accordingly.

\subsubsection{Validation of the code}

One of the major problems connected with numerical simulations is the validation of the code, in particular the tuning of numerical parameters such as the artificial viscosity. The latter is needed to handle shock waves, but on the other hand leads to a damping of physical instabilities, thus possibly introducing artefacts.

Apart from standard tests, as, e.g., the Noh shock tube problem (Noh 1987), the code is required to reproduce correctly the results of an independent linear stability analysis (see chapter 3): Once the code is given an unstable hydrostatic stellar envelope model as initial condition, the result of the integration has to resemble the predetermined growing mode or a superposition thereof, without any further external perturbation. As long as the amplitudes are sufficiently small, i.e., in the linear regime of exponential growth, growth rates and periods of unstable modes provided by the simulation have to be identical with the eigenvalues determined by the independent linear stability analysis.

This requirement strongly reduces the range of admissible numerical parameters and guarantees that the result of the simulation is caused by the physical rather than by a numerical instability or by inconsistencies in the initial conditions. 


\section{Part II}

\section{Results}





\section{Luminous blue variables}

\subsection{Hydrostatic envelope models}

In this section, two envelope models for LBV's will be discussed. Their positions in the HR diagram have been chosen to match the parameters of the prominent LBV $\mathrm{P}$ Cygni, and of an LBV in an eruption phase located above the HD limit (cf. Figure 1.2). Figure 5.1 shows an HR diagram, indicating the position of the models considered by $(\times)$. The evolutionary tracks are labelled with the masses of the corresponding zero age main sequence (ZAMS) stars.

In Table 5.1 the stellar parameters used for the P Cygni model and the eruptive LBV model (M50) are presented. Luminosity, temperature and chemical composition for $\mathrm{P}$ Cygni have been adopted from Pauldrach and Puls (1990) and Najarro et al. (1997). The mass of P Cygni is highly uncertain. While stellar evolution calculations indicate a mass of about $50 M_{\odot}$ (El Eid \& Hartmann 1993), observational data imply $M \approx 23 M_{\odot}$ (Pauldrach \& Puls1990, Najarro, Hillier \& Stahl 1997). For our study we have adopted a value for the mass of P Cygni close to that supported by the observations. The prevailing mass of the eruptive LBV model M50 was taken from stellar evolution calculations.

In Figure 5.2 the internal structure of the envelope model of P Cygni is shown. Temperature $T$ (a) density $\rho$ (b), the ratio $L_{\text {con }} / L$ of convective to total luminosity (c), the

Table 5.1: Stellar parameters for the models considered. In addition to the luminosity $L$, the mass $M$, the effective temperature $T_{\text {eff }}$ and the chemical composition $(X, Y, Z)$ the stellar radius $R$, the ratio of envelope mass to total mass $M_{\text {env }} / M$, the dynamical timescale $\tau_{\text {dyn }}$, the escape velocity $v_{\text {esc }}$ and the Eddington factor $\Gamma$ are given too.

\begin{tabular}{ccccccc} 
& $\log L / L_{\odot}$ & $M / M_{\odot}$ & $T_{\text {eff }}[K]$ & $X$ & $Y$ & $Z$ \\
\hline P Cyg & 5.88 & 26.5 & 19300 & 0.31 & 0.67 & 0.02 \\
M50 & 6.07 & 50 & 10000 & 0.70 & 0.28 & 0.02 \\
& & & & & & \\
& $R\left[10^{12} \mathrm{~cm}\right]$ & $M_{\text {env }} / M$ & $\tau_{\text {dyn }}[\mathrm{sec}]$ & $v_{\text {esc }}\left[\mathrm{cm} \mathrm{sec}^{-1}\right]$ & $\Gamma$ & \\
\hline P Cyg & 5.41 & $4.3 \cdot 10^{-3}$ & $1.2 \cdot 10^{5}$ & $2.29 \cdot 10^{7}$ & 0.43 & \\
M50 & 25.23 & $3.9 \cdot 10^{-2}$ & $8.5 \cdot 10^{5}$ & $3.36 \cdot 10^{7}$ & 0.36 &
\end{tabular}




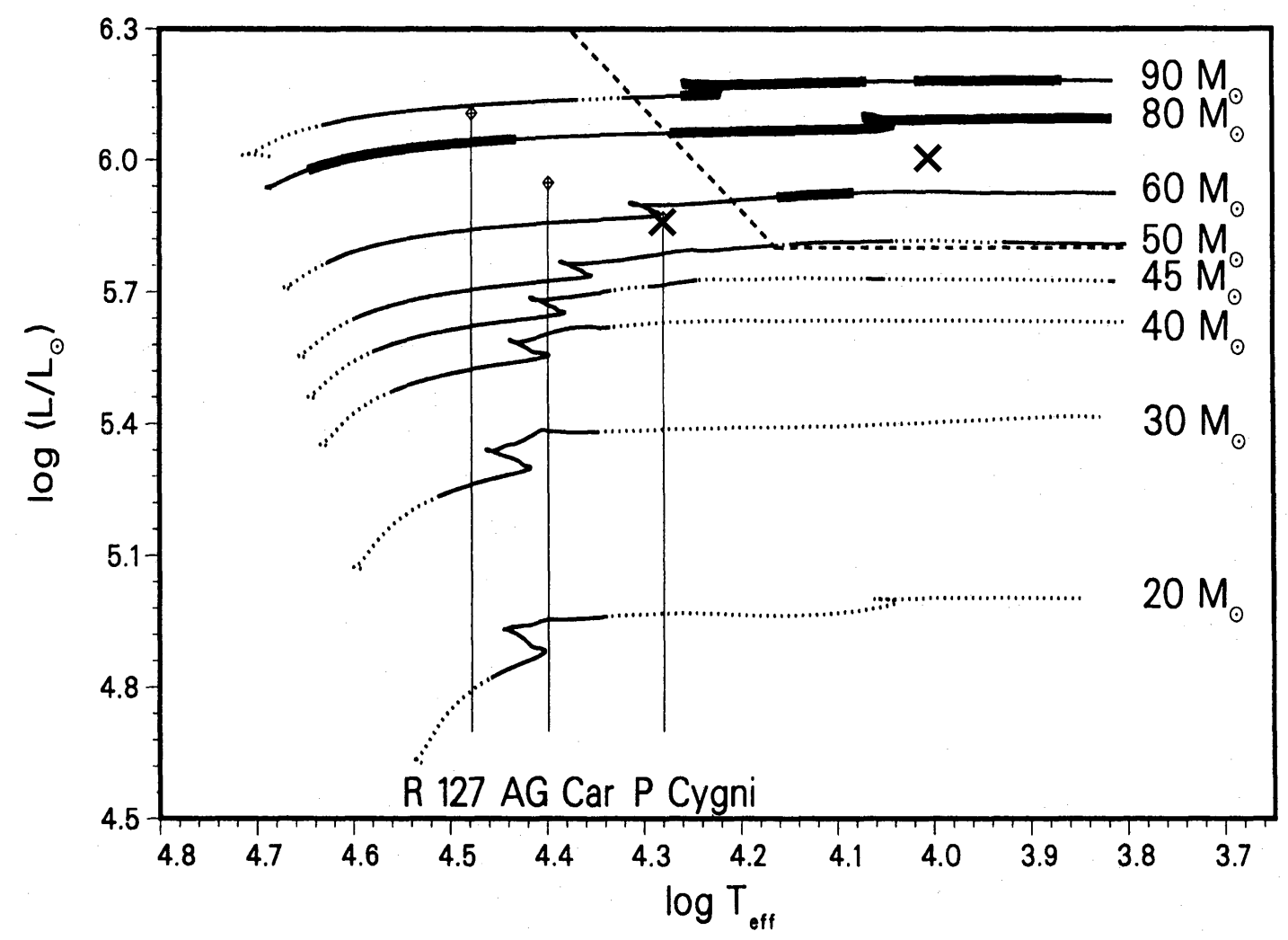

Figure 5.1: HR diagram containing the evolutionary tracks of eight stars (dotted lines) with the initial (ZAMS) masses indicated and the initial chemical composition $(X, Y, Z)=(0.7,0.28,0.02)$, except for the $90 M_{\odot}$ track which corresponds to the initial chemical composition $(X, Y, Z)=(0.73,0.26,0.01)$. Unstable phases are denoted by solid lines and thick lines correspond to dynamical growth rates $\sigma_{i}<-0.1$. Together with the observed positions of R 127, AG Car and P Cyg the location of the HD limit is shown as a dashed line. The positions of the envelope models studied are indicated by $(\times)$. This figure is taken from Kiriakidis, Fricke \& Glatzel, 1993.

ratio $\tau_{\mathrm{th}} / \tau_{\mathrm{dyn}}$ of thermal to dynamical timescale $(\mathrm{d})$, the ratio $\beta$ of gas pressure to total pressure (e) and the sound speed $c_{s}$ (f) are presented as a function of relative radius. The model exhibits a pronounced core-envelope structure, indicated by the steep gradients of temperature and density around $r / R \approx 0.15$. Only 0.43 per cent of the stellar mass are contained in the envelope. A density inversion occurs around $r / R \approx 0.95$. We note that the stratification containing a density inversion is not unstable here, since it is located in a convection zone. The corresponding buoyancy driven instability has already been taken into account within the treatment of convection (see the discussion in section 1.2). At most 40 per cent of the luminosity are transported by convection.

From Figure 5.2 we deduce, that $\tau_{\text {therm }} / \tau_{\text {dyn }}$ is small for a significant fraction of the envelope, implying that the adiabatic approximation in not valid there (cf. section 1.2). 

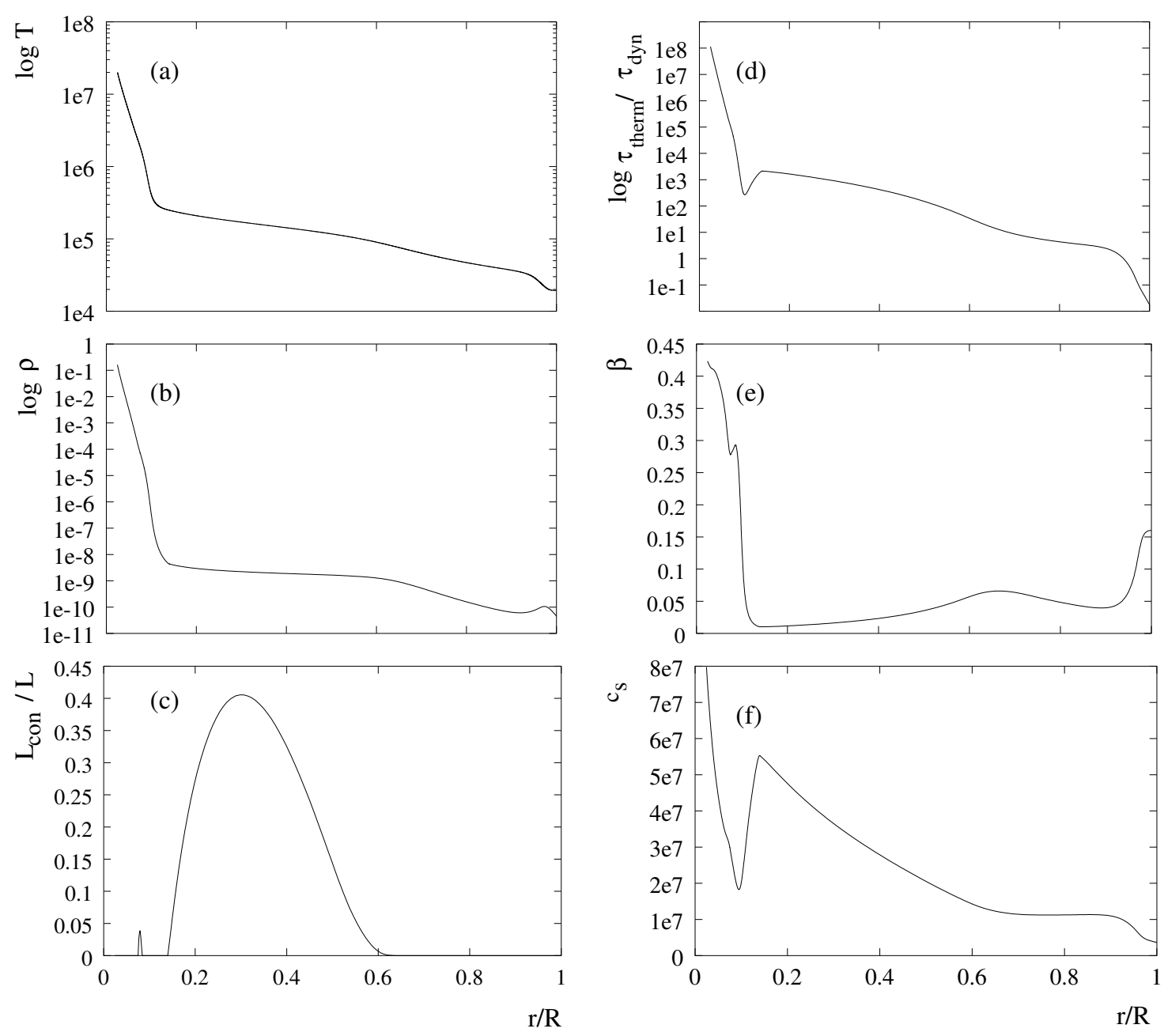

Figure 5.2: Temperature $T$ and density $\rho$ (cgs units), the ratio $L_{\text {con }} / L$ of convective to total luminosity, the ratio $\tau_{\mathrm{th}} / \tau_{\mathrm{dyn}}$ of thermal to dynamical timescale, the ratio $\beta$ of gas pressure to total pressure and the sound speed $c_{s}$ (cgs units) as a function of relative radius for the envelope model of $\mathrm{P}$ Cygni.

Rather the non-adiabatic reversible (NAR, see Gautschy \& Glatzel 1990b) or zero thermal timescale approximation is a much better approximation in the envelopes of LBVs (see section 3.1.2). For $r / R \leq 0.1$ changes of state are adiabatic.

The ratio of gas pressure to total pressure $\beta=\frac{p_{\text {gas }}}{p}$ is pretty small for the major part of the stellar envelope, implying that radiation pressure is dominant and thus favouring strange-mode instabilities. The sound speed exhibits a pronounced maximum around $r / R \approx 0.2$. It is related to the opacity peak due to the contribution of heavy elements and partially separates the stellar envelope into two acoustic cavities. The two sets of modes thus generated are involved in the occurrence of strange modes and associated instabilities (see section 3.3).

The eruptive LBV model M50 exhibits a similar structure as the model for P Cygni 

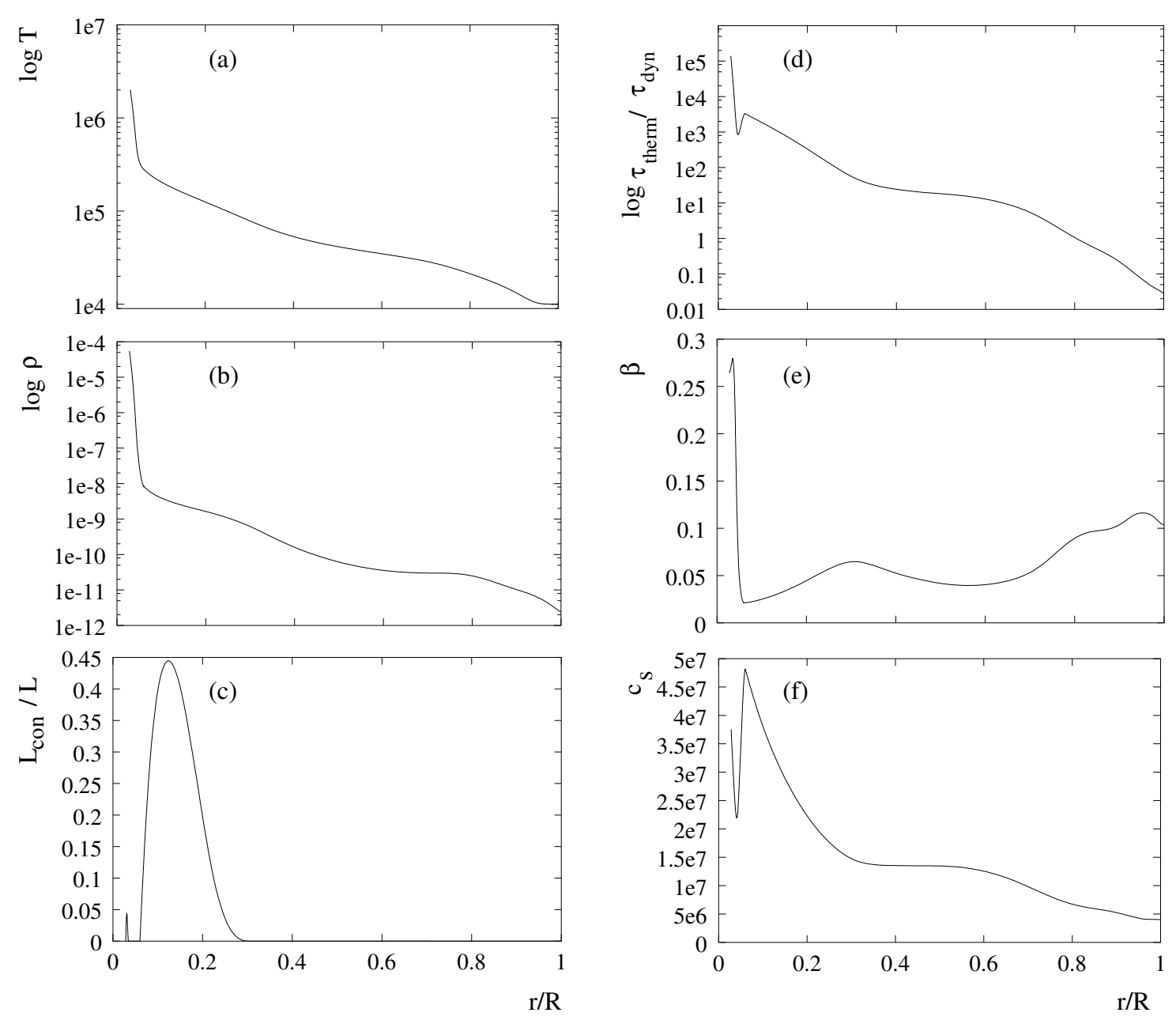

Figure 5.3: Same as Figure 5.2, but for the eruptive LBV model M50.

(Figure 5.3). Here the stellar core contains 96.1 per cent of the total mass and at most 45 per cent of the total luminosity are transported by convection. No density inversion is present.

\subsection{Linear stability analysis}

Using the method described in section 3.4 the lowest order eigenmodes for the LBV models considered in this study have been calculated. The variation of the frequencies has then been followed continuously from the exact treatment to the NAR approximations by switching off the irreversible term in equation $3.17(\Phi \rightarrow 0)$. The results are presented in Figure 5.4 for the eruptive LBV model M50 and the model for P Cygni. Real $\left(\sigma_{R}\right)$ and imaginary $\left(\sigma_{I}\right)$ parts of the eigenfrequencies are given there as a function of the parameter $\Phi$, where $\Phi=1$ corresponds to the exact problem and $\Phi=0$ to the NAR approximation. 

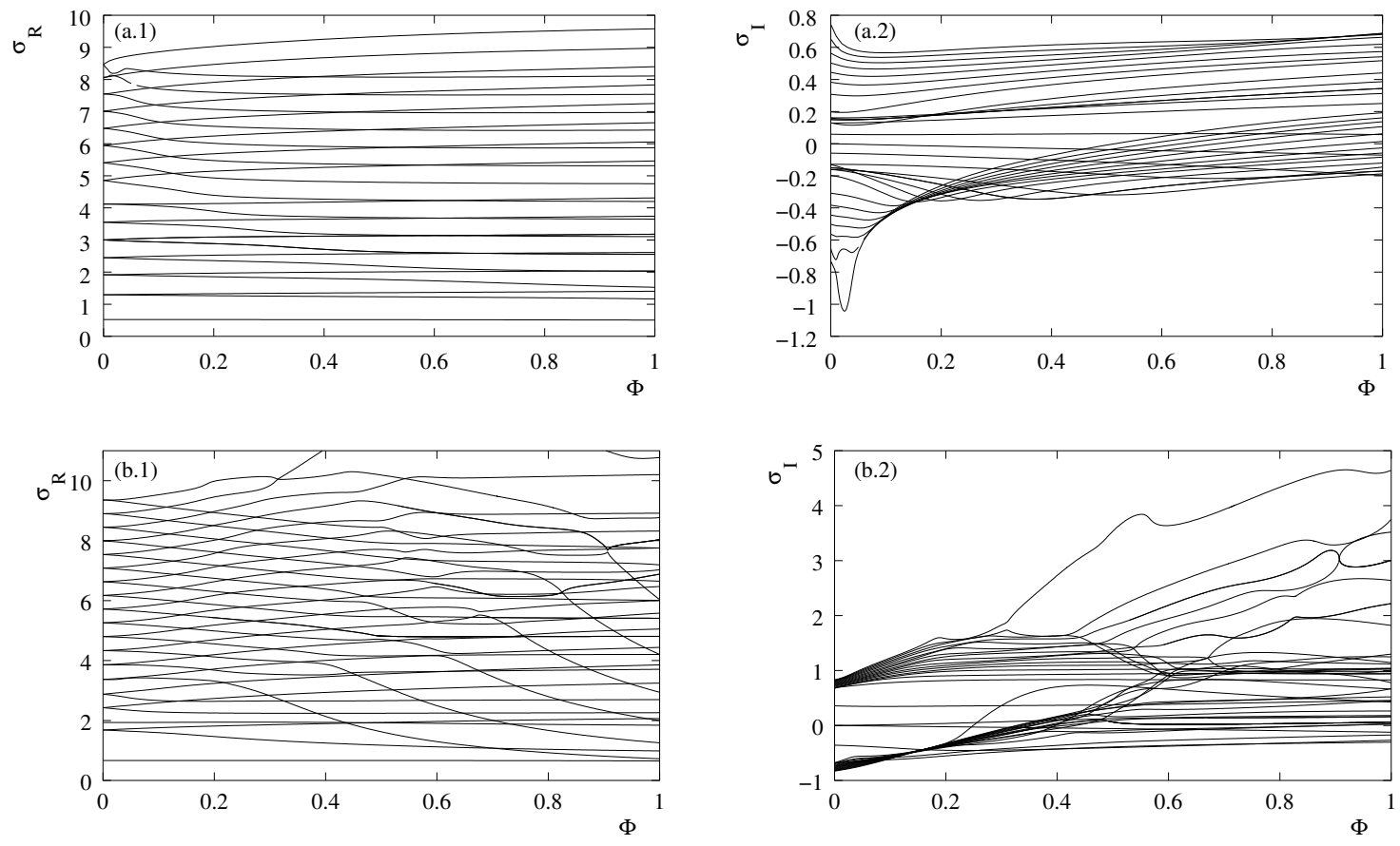

Figure 5.4: Lowest order eigenmodes for the model M50 (a) and the model of P Cygni (b). Real $\left(\sigma_{R}\right)$ and imaginary $\left(\sigma_{I}\right)$ parts of the eigenfrequency $\sigma$ (normalised to the global free fall time) are shown as a function of the parameter $\Phi$. $\Phi=0$ and $\Phi=1$ correspond to the NAR limit and the exact problem, respectively.

For the model M50 (Figure 5.4.a) the real part of the eigenfrequencies varies only slightly for a large range of the parameter $\Phi$. Small changes in $\sigma_{R}$ occur only for values $\Phi<0.2$. When switching off the irreversible term, the imaginary parts of the eigenfrequencies decrease due to a reduced damping, i.e., the modes become more unstable. Below $\Phi \approx 0.2$ changes in the growth and damping rates become more pronounced, which is due to the crossings of the real parts of the eigenfrequencies. Local minima of the imaginary parts can in general be attributed to the crossings of the real parts of the eigenfrequencies. Note, that according to the general properties of the NAR approximation for $\Phi=0$ the eigenvalues form complex conjugate pairs. In the exact problem, only eight modes were found to be unstable with growth rates $\left|\sigma_{I}\right| \propto \mathcal{O}(0.1)$, whereas at least 14 modes modes are unstable with higher growth rates in the NAR approximation. Thus the NAR limit yields only qualitatively correct results.

The counterpart of Figure 5.4.a for the model of P Cygni is shown in Figure 5.4.b. Contrary to M50, mode interactions already take place for values of $\Phi$ close to unity with pronounced variations both in the real and imaginary parts of the eigenfrequencies. Similar to M50 the general trend of decreasing imaginary parts for $\Phi \rightarrow 0$ is recovered. Examples for mode interaction by an instability band and an avoided crossing may be found at $\Phi \approx 0.3, \sigma_{R} \approx 10, \sigma_{I} \approx 1.9$ and $\Phi \approx 0.9, \sigma_{R} \approx 7.5, \sigma_{I} \approx 3$, respectively. In the exact treatment four unstable modes have been identified. 

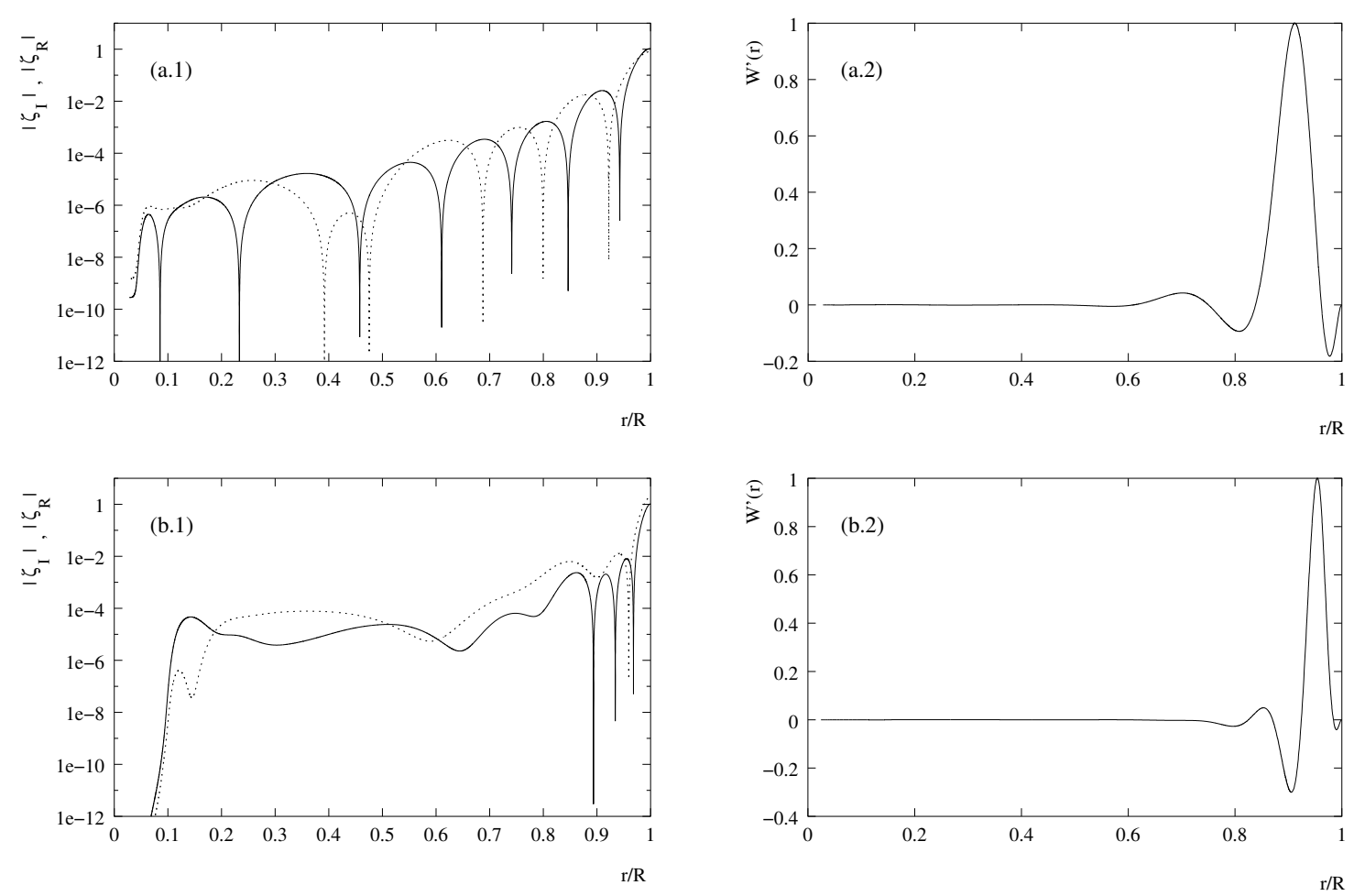

Figure 5.5: Real parts ( $\zeta_{R}$, solid lines) and imaginary parts $\left(\zeta_{I}\right.$, dashed lines) of the Lagrangian displacement (1) and the differential work integral $W^{\prime}(2)$ as a function of relative radius for the eigenmode having $\sigma_{R}=2.02$ and $\sigma_{I}=-0.18$ of the model M50 (a), and for the eigenmode having $\sigma_{R}=2.69$ and $\sigma_{I}=-0.17$ of the model for P Cygni (b).

\subsubsection{Eigenfunctions and work integrals}

In this section the most unstable eigenmodes of M50 and the model for P Cygni will be discussed. Figure 5.5 shows real and imaginary parts of the Lagrangian displacement component of the eigenfunction (left panels) and the corresponding differential work integrals (right panels) as a function of relative radius for M50 and the model of P Cygni, respectively. The differential work integral is given by

$$
\begin{aligned}
W^{\prime} & =\frac{d}{d r} \int d V \frac{1}{\bar{\rho}} \mathfrak{I m}\left(\Delta p^{\dagger} \Delta \rho^{\dagger *}\right) \\
& =4 \pi r^{2} \bar{p} \mathfrak{I m}\left(\frac{\Delta p^{\dagger}}{\bar{p}} \frac{\Delta \rho^{\dagger *}}{\bar{\rho}}\right)
\end{aligned}
$$

and determines whether a region of the star has a driving or damping influence (see section 3.2 and appendix B). Positive values of the differential work integral correspond to driving, whereas negative values correspond to damping. Eigenfunctions are determined only up to a complex constant. This ambiguity is transferred to the work integrals and 
therefore the differential work integrals shown here have been normalised to their maxima. We note that the sign of the integral of $W^{\prime}(r)$ determines whether a mode is stable $(W<0)$ or unstable $(W>0)$.

The Lagrangian displacement corresponding to the eigenvalue $\sigma=2.02-0.18 i$ for the model M50 (Figure 5.5.a) rapidly decreases towards the centre of the star. At $r / R=0.9$ its amplitude has already dropped by two orders of magnitude. The kinetic energy contained in the perturbations being proportional to $|\zeta|^{2}$ has then dropped by four orders of magnitude which justifies the restriction of the analysis to envelope models. The interior of the star remains largely unaffected by the instability. In the non-adiabatic analysis an arrangement scheme for eigenvalues and eigenfunctions does not exist. Therefore node-counting of the eigenfunctions is irrelevant.

The oscillatory character of $W^{\prime}(r)$ is typical for strange modes (see Glatzel 1994). Although the driving indicated by the differential work integral around $r / R \approx 0.9$ coincides with the HeII-ionisation zone, it is not caused by the $\kappa$-mechanism. The latter would be the standard conclusion but is erroneous. Rather the low value of $\beta$ found there simultaneously is the cause of the instability, which is proven by the fact that the mode remains unstable in the NAR-limit (see Figure 5.4).

The Lagrangian displacement corresponding to the eigenvalue $\sigma=2.69-0.17 i$ of the model for P Cygni (Figure 5.5.b) shows a behaviour similar to that found for the eigenvalue $\sigma=2.02-0.18 i$ of M50. Due to the higher effective temperature, the HeIIionisation zone is located closer to the stellar surface than for M50. Accordingly, the driving region associated with the low value of $\beta$ lies also closer to the photosphere.

\subsection{Non-linear evolution of instabilities}

Using the numerical method described in chapter 4, the evolution of the strange-mode instabilities identified in section 5.2 was followed into the non-linear regime. For the simulations unstable hydrostatic envelope models have been used as initial conditions without any further additional external excitation. The instabilities transfer mass from the instability region to the outer parts of the envelope and thus reduce the grid resolution in the inner envelope. The resolution of the instability region, however, severely affects the final result of the instability. Therefore, the simulations had to be stopped if the resolution became insufficient to resolve the unstable regions of the envelope adequately.

Figure 5.6 shows the evolution of the unstable model M50. A sequence of snapshots of the density distribution of the stellar envelope corresponding to time intervals of $\sim 8$ days is presented. The edge of the stellar core is located at $r / R_{0} \approx 0.07$.

By the instability sound waves are generated in the inner envelope. These waves steepen to form shocks, which travel outwards and successively inflate the envelope. The shocks are characterised by a steep density gradient (see, e.g., at time $t \approx 2.6 \cdot 10^{7}$ sec around $r / R_{0} \approx 1$ ). Thus the envelope is inflated to 2.4 initial radii $\left(t \approx 4.0 \cdot 10^{7}\right)$. In this phase the velocities at the stellar surface reach 40 per cent of the escape velocity. Around $t=4 \cdot 10^{7} \mathrm{sec}$ the envelope starts to recollapse and the shock situated at $r / R_{0} \approx 1.1$ is 
stalled. Subsequently this shock is captured in the hydrogen ionisation zone and varies its mean position only slightly by $0.2 r / R_{0}$ on the dynamical timescale. Following shocks emerging from the instability region do not penetrate this structure. The captured shock becomes unstable itself and starts to oscillate rapidly on a timescale comparable to the sound travel time across the width of the shock.

Figure 5.7 shows the evolution of instabilities in the model for P Cygni. Here the edge of the stellar core is located at $r / R_{0} \approx 0.1$. Similar to M50 outwards travelling shock waves successively inflate the envelope. A sequence of shocks located at $r / R_{0} \approx 0.9$, 1.2 and 1.9. may be identified in the snapshot at time $t=4.9 \cdot 10^{7} \mathrm{sec}$. In this phase of the evolution, velocity amplitudes of 55 per cent of the escape velocity are reached. Around $t \approx 7 \cdot 10^{7} \mathrm{sec}$, when the outer envelope still expands and the inner envelope is already falling inwards again, a shock is captured in the hydrogen ionisation zone. As for M50 the captured shock is unstable and starts to oscillate rapidly. Subsequent shocks emerging from the instability region do not pass the captured shock. 

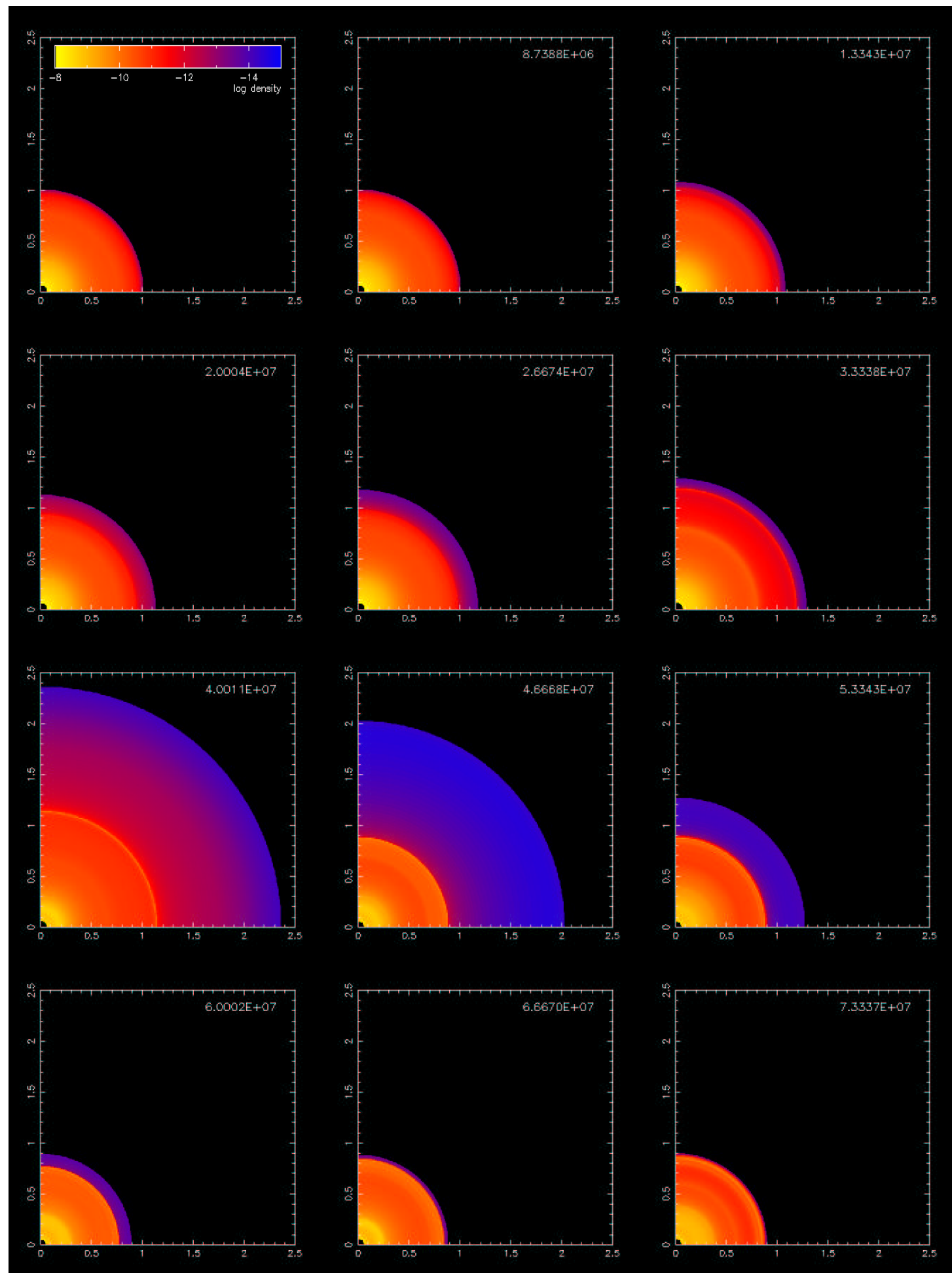

Figure 5.6: Non-linear evolution of instabilities in the envelope of the eruptive LBV model M50. Presented is a sequence of snapshots of the colour coded density distribution (cgs units) at the times [sec] indicated. The time interval between subsequent snapshots corresponds to $\sim 8$ days. Unit of length is the initial radius. 

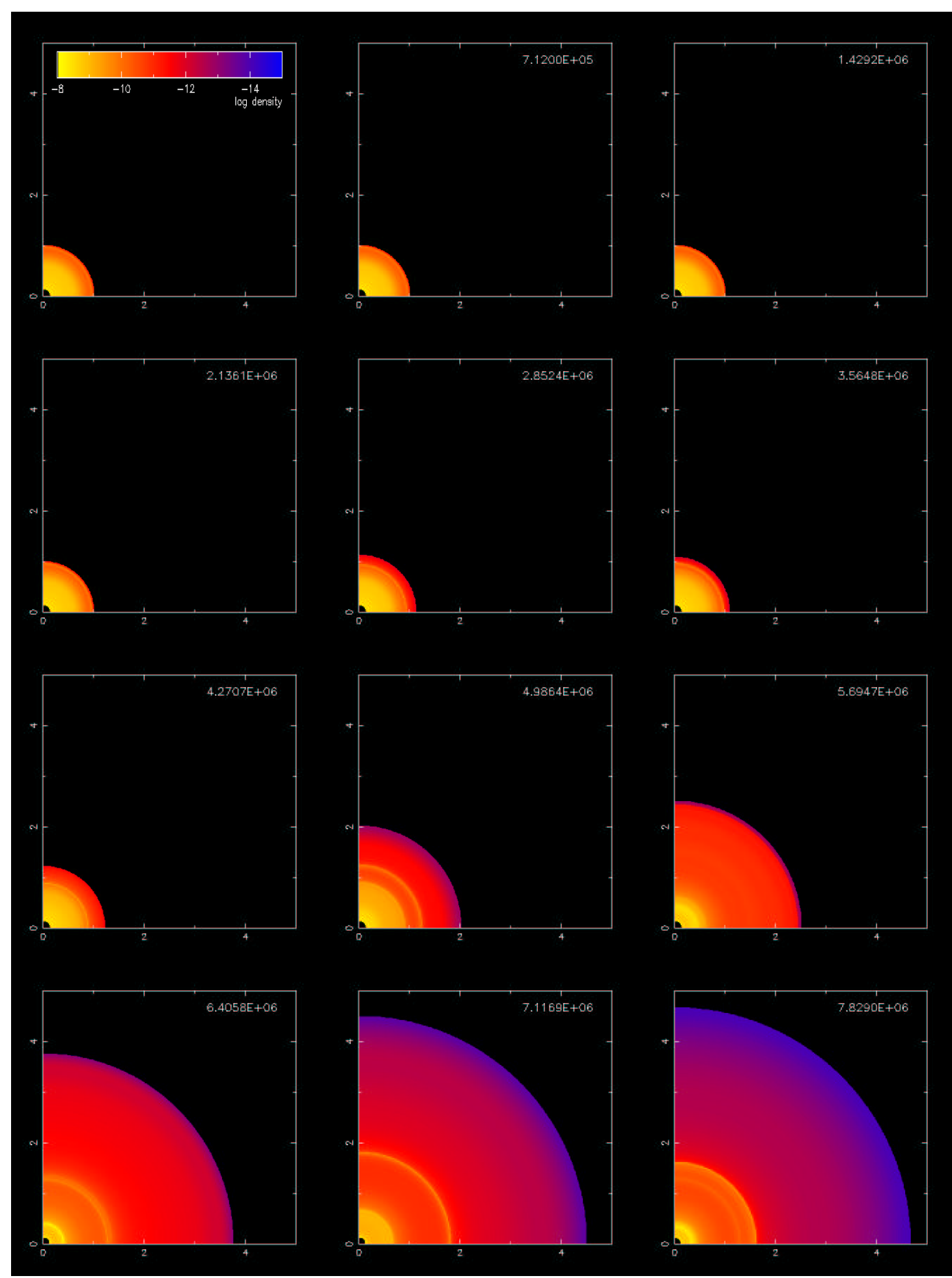

Figure 5.7: Same as Figure 5.6, but for the model of P Cygni. 
6. Captured shocks

\title{
Instabilities of captured shocks in the envelopes of mas- sive stars $^{1}$
}

\author{
M. Grott ${ }^{2}$, W. Glatzel ${ }^{2}$, and S. Chernigovski ${ }^{3}$ \\ ${ }^{2}$ Universitäts-Sternwarte Göttingen, Geismarlandstr .11, 37083 Göttingen, Germany \\ ${ }^{3}$ Institut für Analysis und Numerik, Universität Magdeburg, Universitätsplatz 2, 39106 Magdeburg, Germany
}

\begin{abstract}
The evolution of strange mode instabilities into the non linear regime has been followed by numerical simulation for an envelope model of a massive star having solar chemical composition, $M=50 M_{\odot}, T_{\text {eff }}=10^{4} \mathrm{~K}$ and $L=1.17 \cdot 10^{6} L_{\odot}$. Contrary to previously studied models, for these parameters shocks are captured in the $\mathrm{H}$-ionisation zone and perform rapid oscillations within the latter. A linear stability analysis is performed to verify that this behaviour is physical. The origin of an instability discovered in this way is identified by construction of an analytical model. As a result, the stratification turns out to be essential for instability. The difference to common stratification instabilities, e.g., convective instabilities, is discussed.
\end{abstract}

Key words: hydrodynamics - instabilities - shock waves - stars: mass-loss - stars: oscillations - stars: variables: other.

\subsection{Introduction}

Massive stars are known to suffer from strange mode instabilities with growth rates in the dynamical range (Kiriakidis, Fricke \& Glatzel 1993, Glatzel \& Kiriakidis 1993). The boundary of the domain in the Hertzsprung-Russel diagram (HRD) where all stellar models are unstable - irrespective of their metallicity -, coincides with the observed Humphreys-Davidson (HD) limit (Humphreys \& Davidson 1979). Moreover, the range

\footnotetext{
${ }^{1}$ This paper has been submitted to MNRAS (Grott et al. 2003a)
} 
of unstable models covers the stellar parameters for which the LBV (luminous blue variable) phenomenon is observed.

The high growth rates of the instabilities indicate a connection to the observed mass loss of the corresponding objects. To verify this suggestion, simulations of their evolution into the non linear regime have been performed. In fact, for selected models Glatzel et al. (1999) found the velocity amplitude to exceed the escape velocity (see, however, Dorfi \& Gautschy 2000).

In this paper we report on a stellar model, which in the HRD is located well above the HD-limit, however, at lower effective temperature than the model studied by Glatzel et al. (1999). As expected, this model turns out to be linearly unstable with dynamical growth rates. When following the non linear evolution of the instabilities, shocks form in the non linear regime. The latter is customary in pulsating stellar envelopes (see, e.g., Christy 1966). Contrary to the "hotter" model studied by Glatzel et al. (1999), however, these shocks are captured by the H-ionisation zone after a few pulsation periods. The captured shock starts to oscillate rapidly with periods of the order of the sound travel time across the $\mathrm{H}$-ionisation zone, while its mean position changes on the dynamical timescale of the primary, strange mode instability. This phenomenon is described in detail in section 3.1. Assumptions and methods on which the calculations are based are given in section 2. We emphasise, that in this publication, we concentrate on the oscillations of the captured shock. The phenomenon of shock capture by H-ionisation itself is not investigated here and will be studied in a separate paper.

Apart from a detailed description of the shock oscillations found by numerical simulation the aim of the present paper consists of identifying their origin. This will be achieved by a linear stability analysis in section 3.2. It excludes a numerical origin and attributes the oscillations to a secondary high frequency instability in the shock zone. To identify the physical origin of the instability an analytical model is constructed in section 4. Our conclusions follow.

\subsection{Basic assumptions and methods}

\subsubsection{Construction of initial model}

We investigate a stellar model having the mass $M=50 M_{\odot}$, chemical composition $X=0.7, Y=0.28, Z=0.02$, effective temperature $T_{\text {eff }}=10^{4} \mathrm{~K}$ and luminosity $L=1.17 \cdot 10^{6} L_{\odot}$. These parameters have been chosen to ensure instability of the model. In the Hertzsprung-Russell diagram (HRD) it lies within the instability region identified by Kiriakidis et al. (1993) (c.f. their figure 2). As only the envelope is affected by the instability, the model was constructed by standard envelope integration using the parameters given above. The stellar core and nuclear energy generation are disregarded. Convection is treated in the standard mixing-length theory approach with 1.5 pressure scale heights for the mixing length. The onset of convection was determined by the Schwarzschild criterion. For the opacities, the latest versions of the OPAL tables 
(Iglesias, Rogers \& Wilson 1992, Rogers \& Iglesias 1992) have been used.

\subsubsection{Linear stability analysis}

Having constructed a hydrostatic envelope model its stability with respect to infinitesimal, spherical perturbations is tested. The relevant equations corresponding to mass, energy and momentum conservation and the diffusion equation for energy transport are given in Baker \& Kippenhahn (1962) (hereafter BKA):

$$
\begin{aligned}
& \zeta^{\prime}=C_{4}\left(3 \zeta+C_{5} p-C_{6} t\right) \\
& l^{\prime}=(i \sigma) C_{1}\left(-p+C_{2} t\right) \\
& p^{\prime}=-\left(4+C_{3} \sigma^{2}\right) \zeta-p \\
& t^{\prime}=C_{7}\left(-4 \zeta+C_{13} l+C_{8} p-C_{9} t\right)
\end{aligned}
$$

$\zeta, l, p$ and $t$ are the relative perturbations of radius, luminosity, pressure and temperature, respectively, and dashes denote derivatives with respect to $\ln p_{0} . \sigma$ is the eigenfrequency normalised to the inverse of the global free fall time $\tau_{\mathrm{ff}}=\sqrt{R^{3} / 3 G M}$. The coefficients $C_{i}$ are determined by the background model where $C_{13}$ denotes the ratio of total and radiative luminosity. The other coefficients are defined in BKA. For the general theory of linear non-adiabatic stability, we refer the reader to Cox (1980) and Unno (1989).

The coupling between pulsation and convection is treated in the standard frozen in approximation, i.e., the Lagrangian perturbation of the convective flux is assumed to vanish. This is justified since the convective flux never exceeds $10 \%$ of the total energy flux. Moreover, the convective timescale is much longer than the dynamical timescale of the pulsations considered. The solution of the perturbation problem has been determined using the Riccati method (Gautschy \& Glatzel 1990). As a result of the linear non adiabatic (LNA) stability analysis we obtain periods and growth or damping rates of various modes together with the associated eigenfunctions.

\subsubsection{Non-linear evolution}

Having identified an instability by the LNA analysis its growth is followed into the nonlinear regime. Assuming spherical symmetry, we adopt a Lagrangian description and choose as independent variables the time $\mathrm{t}$ and the mass $m$ inside a sphere of radius $r$. The evolution of an instability is then governed by mass conservation,

$$
\frac{\partial r^{3}}{\partial m}-\frac{3}{4 \pi \rho}=0
$$

momentum conservation,

$$
\frac{\partial^{2} r}{\partial t^{2}}+4 \pi r^{2} \frac{\partial P}{\partial m}+\frac{G m}{r^{2}}=0
$$


energy conservation,

$$
\frac{\partial L}{\partial m}-\frac{P}{\rho^{2}} \frac{\partial \rho}{\partial t}+\frac{\partial E}{\partial t}=0
$$

and the diffusion equation for energy transport,

$$
\frac{\partial T}{\partial m}-\frac{3 \kappa\left(L-L_{k o n v}\right)}{64 \pi^{2} a c r^{4} T^{3}}=0
$$

where $\rho, p, T, L$, and $E$ denote density, pressure, temperature, luminosity and specific internal energy, respectively. $a$ is the radiation constant, $c$ the speed of light and $G$ the gravitational constant. For consistency, the equation of state $p(\rho, T)$ and the opacity $\kappa$ are identical with those used for the construction of the initial model. In accordance with the LNA stability analysis, convection is treated in the frozen in approximation, i.e., $L_{k o n v}$ is taken to be constant during the non-linear evolution and equal to the initial value. For the treatment of shocks artificial viscosity is introduced by substituting $P=P+Q$ with ( $v$ is the velocity)

$$
Q= \begin{cases}C_{0} \rho(\operatorname{div} v)^{2} & \operatorname{div} v<0 \\ 0 & \operatorname{div} v \geq 0\end{cases}
$$

and $C_{0}>0$ (von Neumann - Richtmyer form of artificial viscosity).

For some difference schemes including the Fraley scheme, which the present method is based on, this form of the artificial viscosity can give rise to undesired, unphysical oscillations (see, e.g., Buchler \& Whalen 1990). To avoid these, artificial tensor viscosity is usually used (Tscharnuter \& Winkler 1979). In order to be sure that the oscillations observed are not caused by the form of the artificial viscosity, we have run tests both with volume and tensor viscosity. As a result, shock oscialltions are found independently for any form of the artificial viscosity. As the von Neumann - Richtmyer viscosity allows for a straightforward formulation of the boundary conditions discussed below, we have for convenience chosen to work with it.

The inert hydrostatic core provides boundary conditions at the bottom of the envelope by prescribing its time independent radius and luminosity there. As the outer boundary of the model does not correspond to the physical boundary of the star, boundary conditions are ambiguous there. We require the gradient of heat sources to vanish there:

$$
\operatorname{grad}(\operatorname{div} F)=0
$$

This boundary condition is chosen to ensure that outgoing shocks pass through the boundary without reflection. The numerical code relies on a Lagrangian, with respect to time implicit, fully conservative difference scheme proposed by Fraley (1968) and Samarskii \& Popov (1969) Concerning tests of the code, we adopted the same criteria as Glatzel et al. (1999). 

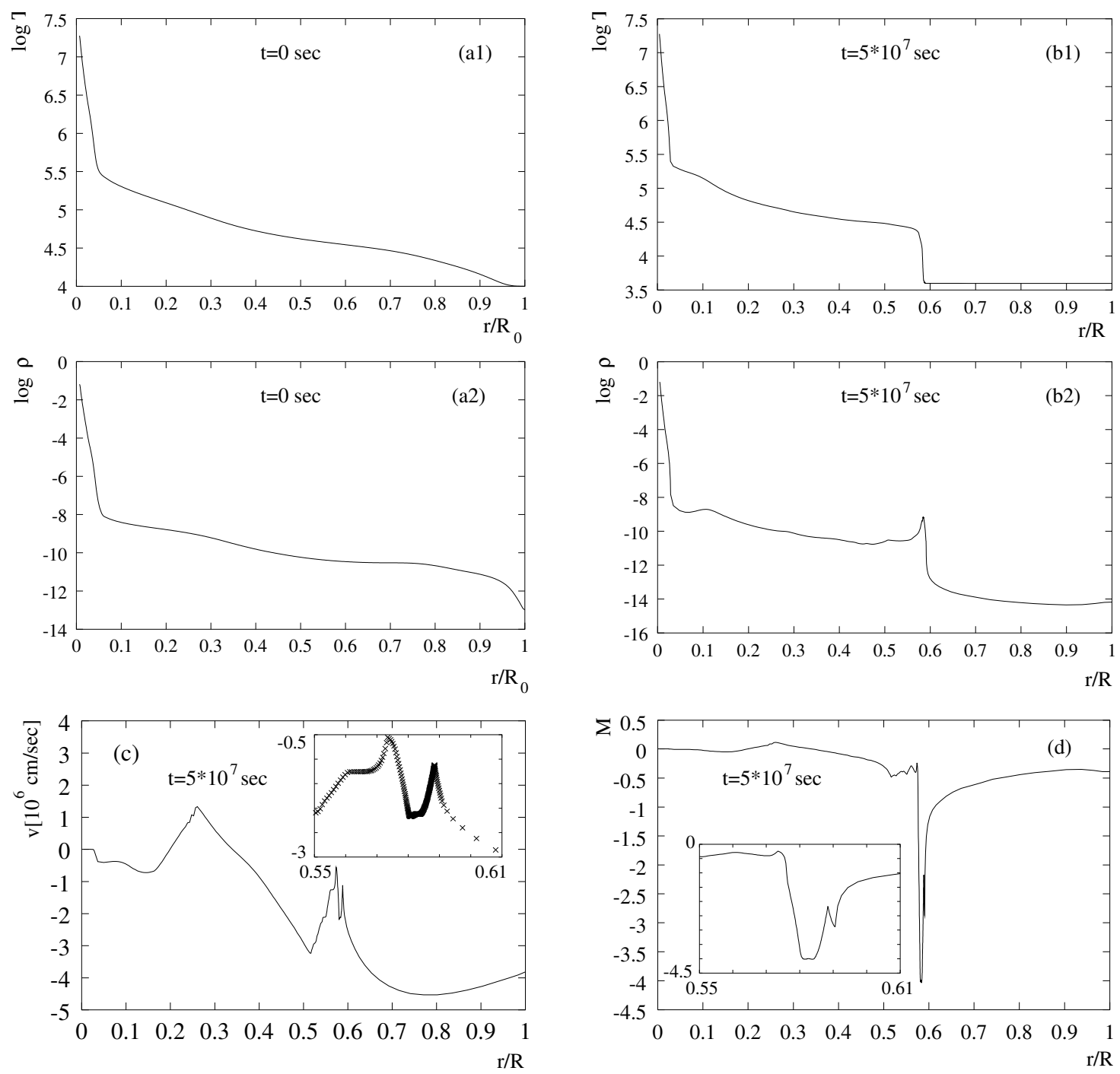

Figure 6.1: Temperature $T$ and density $\rho$ as a function of relative radius of the initial model (a1-a2), and the model at $5 \cdot 10^{7} \mathrm{sec}$ (b1-b2). Velocity $v$ and Mach number $M$ are shown as a function of relative radius for the model at $5 \cdot 10^{7} \mathrm{sec}$ in (c) and (d), respectively

\subsection{Numerical results}

\subsubsection{The evolution of the stellar model}

Density and temperature of the initial model as a function of relative radius, are shown in figures 6.1.a1-6.1.a2. The stratification exhibits a pronounced core-envelope structure, which is typical for stellar models in this domain of the HRD. More than 96 per cent of the mass is concentrated in the core, which extends to less than 5 per cent of the total radius. It remains in hydrostatic equilibrium and is not affected by the instability. 
Table 6.1: Unstable modes of the initial model. $\sigma_{r}$ denotes the real part and $\sigma_{i}$ the imaginary part of the eigenfrequency $\sigma$ normalised by the global free fall time.

\begin{tabular}{rrrrrrrr}
\hline$\sigma_{r}$ & 0.53 & 1.22 & 1.66 & 2.12 & 2.26 & 3.34 & 3.86 \\
$\sigma_{i}$ & -0.06 & -0.18 & -0.13 & -0.20 & -0.12 & -0.04 & -0.04 \\
\hline
\end{tabular}

The initial model has been tested for stability and been found unstable on dynamical timescales. This instability will be referred to as primary instability hereafter. With respect to its physical origin, it is a strange mode instability, which has been identified in a variety of stars including Wolf-Rayet-stars, HdC-stars and massive stars (like the present model). Strange modes appear as mode coupling phenomena with associated instabilities whenever radiation pressure is dominant. The latter is true for a large fraction of the radius in the present model. The linear stability analysis of the initial model reveals several unstable modes. Eigenfrequencies of the most unstable ones, i.e., their real $\left(\sigma_{r}\right)$ and imaginary parts $\left(\sigma_{i}\right)$, are presented in table 6.1 .

The evolution of the linear instabilities was followed into the non-linear regime by numerical simulation using the hydrostatic model as initial condition. No additional initial perturbation of the hydrostatic model was added. Rather the code was required to pick the correct unstable modes from numerical noise. By comparing growth rates and periods obtained in the simulation with the results of the LNA analysis, the linear regime of the evolution was used as a test for the quality of the simulation.

In the non-linear regime sound waves travelling outwards form shocks and initially inflate the envelope to 2.5 initial radii. Thus velocity amplitudes of $10^{7}[\mathrm{~cm} / \mathrm{sec}]$ are reached. One of the subsequent shocks is captured around the $\mathrm{H}$-ionisation zone at relative radius $r / R=0.58$ and $3.6<\log T<4.7$. The mechanism responsible for the shock capturing will not be studied in this publication. Rather, we will investigate the oscillations on the shock front and show that they are of physical origin. A snapshot at time $t=5 \cdot 10^{7} \mathrm{sec}$ of the situation containing the captured shock is shown in Figures 6.1.b1 and 6.1.b2. Figure 6.1.c shows the velocity as a function of relative radius at this instant. Sound waves are generated in the region around $r / R \approx 0.1$ and travel outwards, growing in amplitude and steepening. In the snapshot one such wave is located at $r / R \approx 0.25$. The captured shock front is located at $r / R \approx 0.58$ and the outer envelope is collapsing onto it. The small panel in Figure 6.1.c shows the details of the region containing the captured shock, indicating the grid resolution by $(\times)$. Within the Lagrangian description, $\sim 150$ of the 512 gridpoints used are concentrated in the shock zone. Figure 6.1.d shows the Mach number $M=v / v_{s}$ as a function of relative radius for the snapshot ( $v_{s}$ is the local sound speed). The Mach number changes by 3.5 across the shock front around $r / R \approx 0.58$.

After the formation of the captured shock its position varies only weakly by $\approx 0.2$ relative radii on the timescale of the primary instability (see figure 6.2.b). Superimposed on this variation is a much faster oscillation, whose timescale is related to the sound 

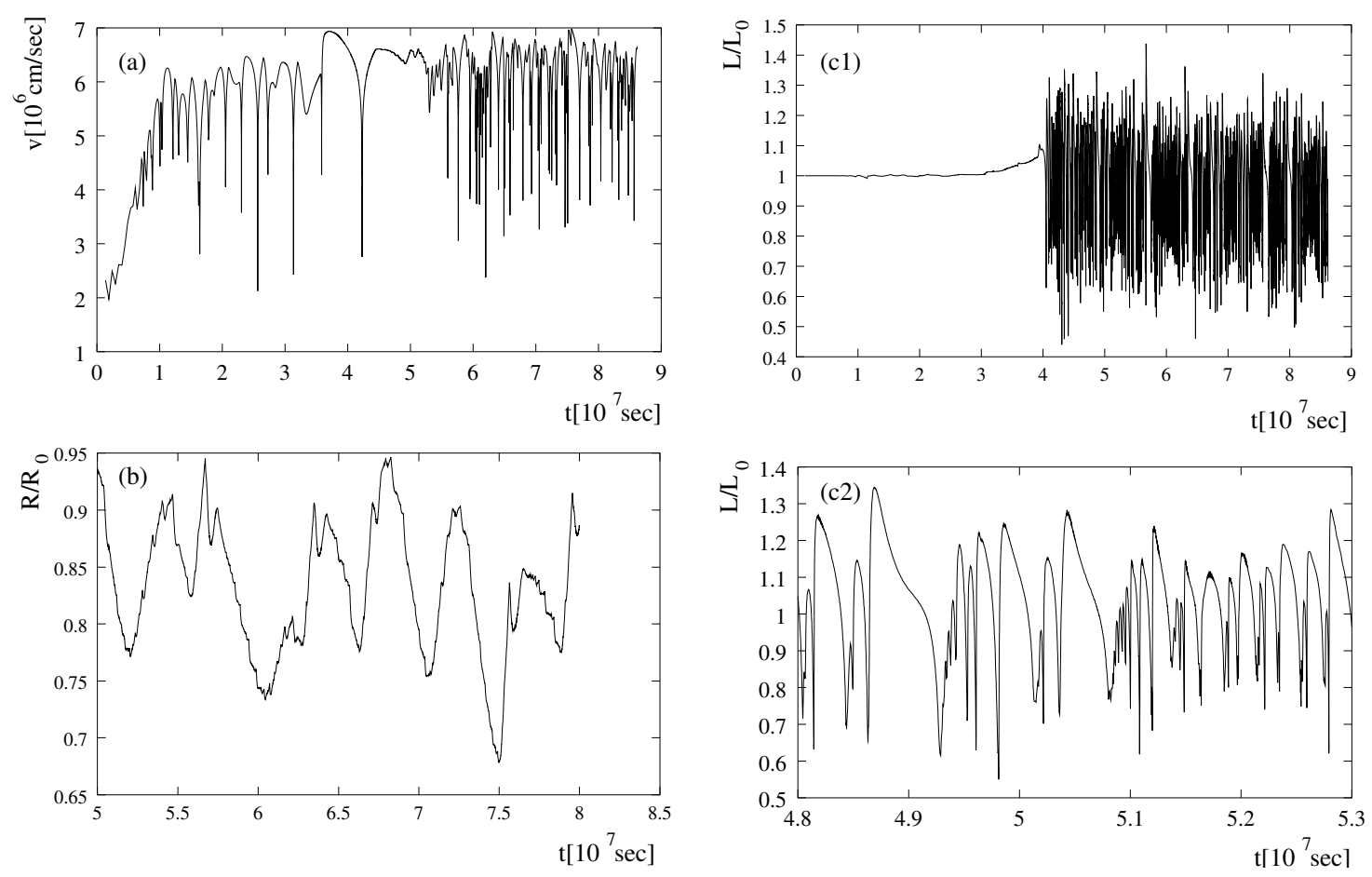

Figure 6.2: The velocity $v$ at the boundary (a), the relative position of the shock front (b) and the relative luminosity $L / L_{0}$ at the boundary on two different scales (c1-c2) as a function of time $t$.

travel time across the shock $\left(\approx 10^{5} \mathrm{sec}\right)$. It is even more pronounced in the run of the luminosity (figure 6.2.c2). The onset of the fast oscillation with the capturing of the shock by the H-ionisation-zone is illustrated in figures 6.2.a and 6.2.c1, where the velocity $v$ and relative luminosity $L / L_{0}$ at the outer boundary are shown as a function of time. Up to $\approx 4 \cdot 10^{7} \mathrm{sec}$ the velocity varies on the timescale of the primary instability and the luminosity remains approximately constant due to the low heat capacity of the envelope of the star. After $\approx 4 \cdot 10^{7} \mathrm{sec}$, when the shock has been captured by the Hionisation-zone, luminosity and velocity vary on the shorter timescale of the secondary shock oscillation. The luminosity perturbation has its origin in the shock. Due to the low heat capacity the luminosity perturbation remains spatially constant above the shock.

In principle, the high-frequency secondary oscillations of the shock could be caused numerically. However, the results are largely independent of the numerical treatment and parameters, which has been verified by extensive numerical experiments suggesting a physical origin of the phenomenon. In section 6.3.2, we shall argue in favour of the latter by presenting a linear stability analysis providing an instability with appropriate frequencies and growth rates. 


\subsubsection{Stability analysis of a model containing a captured shock}

In this Section we shall initially assume and then prove a posteriori, that the secondary oscillations of the captured shock described in section 6.3.1 are caused by physical processes. We perform a linear stability analysis of a background model by assuming that the dependent variables radius, pressure, temperature and luminosity may be expressed as the sum of a background contribution and a small perturbation:

$$
x(m, t)=x_{0}(m, t)+x_{1}(m, t) \quad \text { for } \quad \mathrm{x} \in\{\mathrm{r}, \mathrm{p}, \mathrm{T}, \mathrm{L}\}
$$

The background coefficients $x_{0}(m, t)$ may be regarded as time independent, i.e., $x_{0}(m, t)=$ $x_{0}(m)$, as long as the perturbations vary on much shorter timescales as the background, i.e., as long as the condition

$$
\frac{d \log x_{0}(m, t)}{d t} \ll \frac{d \log x_{1}(m, t)}{d t}
$$

holds. $\frac{d}{d t}$ denotes the Lagrangian time derivative. Thus, the variations on dynamical timescales of the model containing the captured shock are regarded as stationary with respect to the anticipated much faster instability. Eigenmodes with periods of the order of the dynamical timescale suffer from the competition with the variation of the "background" model, whereas the approximation holds for those with much shorter periods. For the model considered, the approximation is correct for $|\sigma|>100$. Should unstable eigenmodes of this kind exist, this would prove the instability and the high frequency oscillations of the captured shocks to be of physical origin. Therefore, the results of a linear stability analysis of such a model are meaningful, as long as the obtained frequencies are interpreted properly.

A problem with this strategy is that the numerical simulations provide only the superposition of the slow dynamical and the secondary, fast oscillations. The linear stability analysis, however, requires a - on the fast oscillations - stationary background model. It is obtained by an appropriate time average over a numerically determined sequence of models. "Appropriate" means, that the average has to be taken over times longer than the short period oscillations and shorter than the dynamical timescale. Thus all physical quantities $Q(m, t)$ are averaged according to

$$
<Q(m)>=\frac{1}{t_{e}-t_{s}} \int_{t_{s}}^{t_{e}} Q(m, t) \mathrm{d} t
$$

where $t_{s}$ and $t_{e}$ are the beginning and the end of the averaging interval and satisfy the requirements discussed above. $t_{s}$ has been varied between $4 \cdot 10^{7} \mathrm{sec}$ and $5 \cdot 10^{7} \mathrm{sec}$ (after the formation of the shock front) and the averaging interval between $5 \cdot 10^{5} \mathrm{sec}$ and $1 \cdot 10^{6}$ sec. All averages exhibit qualitatively the same behaviour and the LNA stability analysis is largely independent of the averaging parameters. The results presented in the following were obtained for $t_{s}=5 \cdot 10^{7} \mathrm{sec}$ and $t_{e}=5.05 \cdot 10^{7} \mathrm{sec}$. 

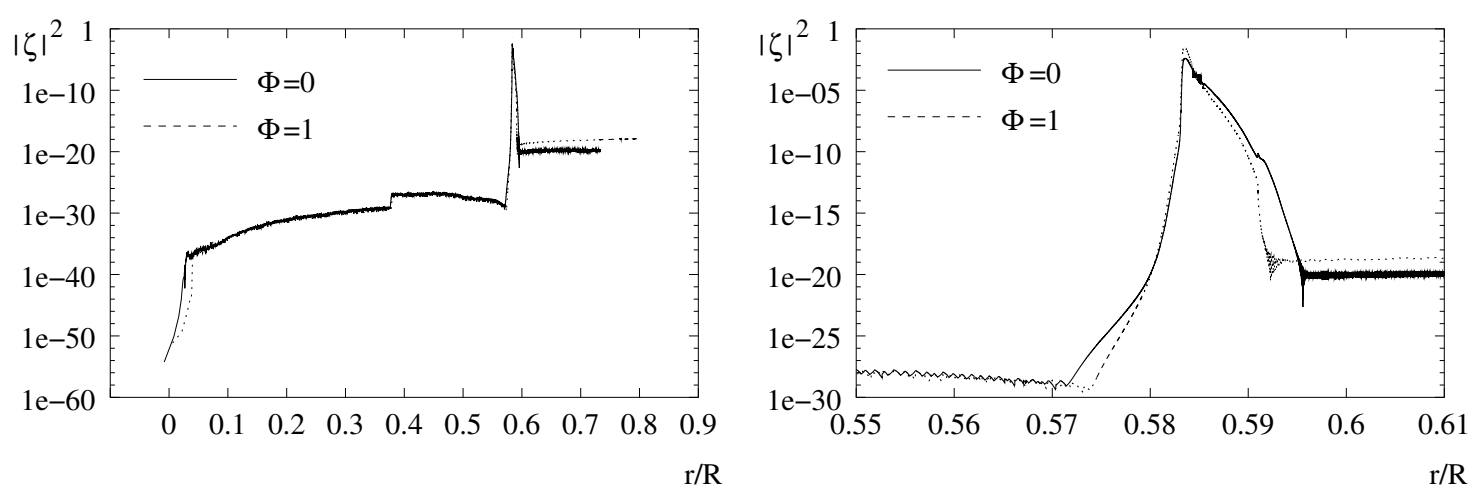

Figure 6.3: Modulus of the Lagrangian displacement $\zeta$ as a function of relative radius for the eigenfrequencies $\sigma_{r}=4651.7, \sigma_{i}=-119.6$ ( $\Phi=0$, solid lines) and $\sigma_{r}=4837.2$, $\sigma_{i}=-83.9$ ( $\Phi=1$, dotted lines). The right panel shows details for the shock zone.

With these assumptions, the linear perturbation equations 6.1-6.4, which have been derived for a strictly static background model, remain valid even for the situation studied here, except for the momentum equation 6.3, which has to be modified according to:

$$
p^{\prime}=-\left(4+C_{3} \sigma^{2}\right) \zeta-Q_{1} p \quad \text { with } \quad Q_{1}=-\frac{\partial p_{0}}{\partial m} \frac{4 \pi r_{0}^{4}}{G m}
$$

$Q_{1} \neq 1$ accounts for the deviations from hydrostatic equilibrium.

As a result of the linear stability analysis (with $Q_{1} \neq 1$ ), the expected unstable modes having high frequencies have been identified. E.g., a typical mode of this kind satisfying the assumptions discussed has the frequency $\sigma_{r}=4837.2$ and the growth rate $\sigma_{i}=$ -83.9 .

In a second step, we investigate the influence of deviations from hydrostatic equilibrium, i.e., the deviations from $Q_{1}=1$. For this purpose we rewrite equation 6.14 as

$$
p^{\prime}=-\left(4+C_{3} \sigma^{2}\right) \zeta-p+\Phi\left(1-Q_{1}\right) p
$$

with $0 \leq \Phi \leq 1$. The limits $\Phi=0$ and $\Phi=1$ correspond to hydrostatic equilibrium and the averaged model containing the shock, respectively. The influence of deviations from hydrostatic equilibrium is then studied by varying $\Phi$ between 0 and 1 . Following the mode having $\sigma_{r}=4837.2$ and $\sigma_{i}=-83.9$ at $\Phi=1$ to $\Phi=0$ its frequency and growth rate changes to $\sigma_{r}=4651.7$ and $\sigma_{i}=-119.6$. The moduli of the corresponding Lagrangian displacements, which indicate the kinetic energy of the pulsations, are shown in Figure 6.3 as a function of relative radius. The energy of the pulsation is concentrated around $r / R \approx 0.58$ and drops off exponentially above and below. As a result, neither eigenvalues nor eigenfunctions differ significantly for $\Phi=0$ and $\Phi=1$, i.e., the assumption of hydrostatic equilibrium is justified for the unstable modes considered. Therefore, we will assume hydrostatic equilibrium in a further discussion and investigation of the secondary instability, i.e., all subsequent results were obtained assuming $\Phi=0$. 
Table 6.2: Unstable modes of the averaged model. $\sigma_{r}$ denotes the real part and $\sigma_{i}$ the imaginary part of the eigenfrequency $\sigma$ normalised by the global free fall time.

\begin{tabular}{rrrrrrrrrrr}
\hline$\sigma_{r}$ & 0.92 & 2.22 & 3.31 & 4.74 & 6.08 & 7.48 & 8.79 & 10.1 & 11.4 & 140.4 \\
$\sigma_{i}$ & -0.05 & -0.33 & -0.32 & -0.25 & -0.29 & -0.18 & -0.21 & -0.24 & -0.12 & -0.1 \\
\hline$\sigma_{r}$ & 154.4 & 162.9 & 157.2 & 179.6 & 202.0 & 413.6 & 447.3 & 487.5 & 541.4 & 4651.7 \\
$\sigma_{i}$ & -0.05 & -35.4 & -0.08 & -27.8 & -17.6 & -30.4 & -26.3 & -54.9 & -0.35 & -119.6 \\
\hline
\end{tabular}

The results of a LNA stability analysis according to equations 6.1-6.4 and Section 6.2.2 for the averaged model are summarised in Table 6.2, where representative values for the eigenfrequencies of unstable modes are given. Three sets of unstable modes may be distinguished. Low order modes with $\sigma_{r}$ between 0.9 and 9 have growth rates of the order of 0.2 , i.e a ratio of $\frac{\sigma_{i}}{\sigma_{r}} \approx 0.1$. They can be identified with the primary instability. However, their periods compete with the variation of the background model and therefore these modes have to be interpreted with caution. The properties of two classes of high order unstable modes with frequencies $\sigma_{r}$ between 140 and 4650 are in accordance with our approximation. One of them has high growth rates with a ratio of $\frac{\sigma_{i}}{\sigma_{r}} \approx 0.1$, the second low growth rates with a ratio of $\frac{\sigma_{i}}{\sigma_{r}} \approx 5 \cdot 10^{-4}$. The latter may be identified with high order primary instabilities, whereas the former are attractive candidates for the secondary, shock front instabilities sought.

For further discussion, we consider eigenfunctions and the corresponding work integrals of representative members of the different sets of modes. The work integral is a widely used tool to identify the regions in a star, which drive or damp the pulsation. Glatzel (1994) has shown, that the concept of the work integral is not necessarily restricted to small values of the damping or growth rate. By replacing the conventional time average by an ensemble average it can be extended to arbitrary values of $\frac{\sigma_{i}}{\sigma_{r}}$. In any case, one arrives at the expression

$$
W(r)=\frac{\sigma_{i}}{\sigma_{r}} \sim \int_{0}^{r} \pi r^{2} \bar{p} \operatorname{Im}\left(p^{\dagger} \rho^{\dagger *}\right) \mathrm{d} r^{\prime}
$$

for the work integral, where $\operatorname{Im}(z)$ denotes the imaginary part of $z,()^{*}$ denotes complex conjugation and $p^{\dagger}, \rho^{\dagger}$ denote the spatial parts of the eigenfunctions of the relative pressure and density perturbations, respectively. $\bar{p}$ is the pressure of the background model. The sign of the integrand in equation 6.16 determines, if a region of the star damps or drives the pulsation, where $\operatorname{Im}\left(p^{\dagger} \rho^{\dagger *}\right)<0$ corresponds to driving and $\operatorname{Im}\left(p^{\dagger} \rho^{\dagger *}\right)>0$ to damping. Some authors (e.g. BKA) use $\log p$ instead of $r$ as independent variable and therefore obtain an opposite sign of the differential work integral for driving and damping influence. To match this convention, $-W(r)$ is shown in figures 6.4.b, i.e., driving regions correspond to positive $-W(r)$, damping regions to negative $-W(r)$.

According to its eigenvalues the mode corresponding to $\sigma_{r}=2.22, \sigma_{i}=-0.33$ was identified as a primary instability. This is supported by the Lagrangian displace- 

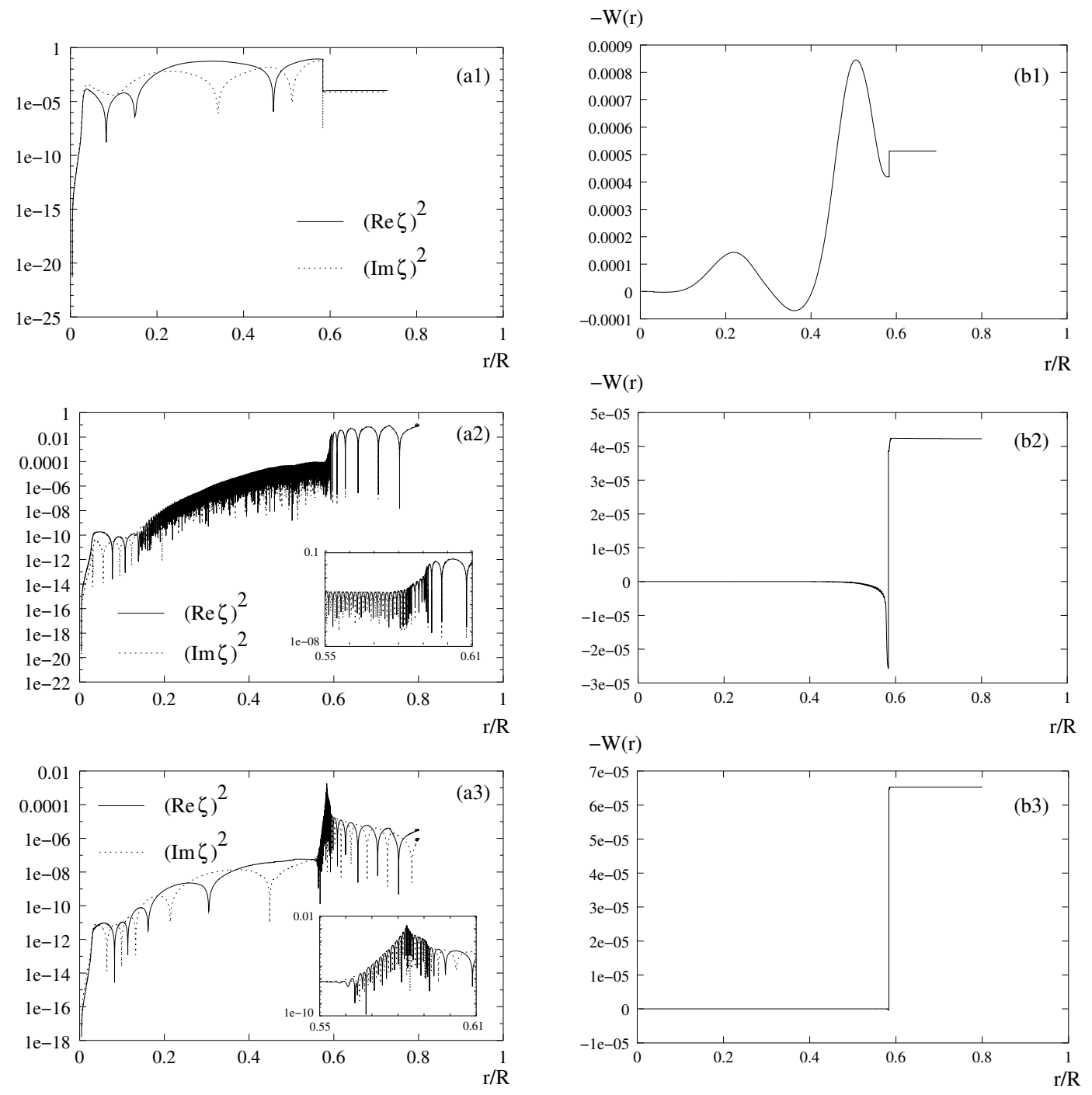

Figure 6.4: Lagrangian displacements $\zeta$ (a) and integrated workintegrals (b) as a function of relative radius for the eigenfrequencies $\sigma_{r}=2.22, \sigma_{i}=-0.33(1), \sigma_{r}=157.2$, $\sigma_{i}=-0.08$ (2) and $\sigma_{r}=162.9, \sigma_{i}=-35.4$ (3) of the averaged model.

ment component $\zeta$ of the eigenfunction and the work integral shown in figure 6.4.a1 and 6.4.b1. The shock front acts as an acoustic barrier causing the eigenfunction to vanish above it (figure 6.4.a1). The work integral (figure 6.4.b1) exhibits two driving regions which coincide with the opacity peaks at $\log T=5.3$ (caused by the contributions of heavy elements) and $\log T=4.7$ (He-ionisation). The stability properties are not affected significantly by the shock region.

The counterparts of figures 6.4.a1 and 6.4.b1 for a weakly unstable high frequency mode having $\sigma_{r}=157.2, \sigma_{i}=-0.08$ are shown in figures 6.4.a2 and 6.4.b2. Again, the isolating effect of the shock front causes the dramatic variation of the amplitude around 
$r / R_{0}=0.6$. However, in contrast to the eigenfunction presented in figure 6.4.a1 the amplitude is now significant above and negligible below the shock. High order modes of this kind in general exhibit strong damping. For the mode considered the shock efficiently screens the inner damping part of the stellar envelope. Thus the region below the shock contributes only weak damping which is overcome by the driving influence of the shock as shown by the work integral in figure 6.4.b2.

Apart from splitting the acoustic spectrum by an acoustic barrier into two sets of modes associated with the acoustic cavities below and above the shock, respectively, the shock itself gives rise to a third set. Lagrangian displacement and work integral for a typical member of this set having $\sigma_{r}=162.9, \sigma_{i}=-35.4$ are shown in figures 6.4.a3 and 6.4.b3. The amplitude of this unstable mode reaches its maximum on the shock and drops off exponentially above and below. Note its oscillatory behaviour on and close confinement to the shock. The real parts of this set of eigenfrequencies of $\approx 200-500$ correspond to periods of $\Pi \approx 8 \cdot 10^{4}-2 \cdot 10^{5}$ sec, which are observed in the luminosity perturbations (cf. figure 6.2.c2) induced by the shock oscillations. The work integral (figure 6.4.b3) shows, that the shock is driving this instability, and that the regions above and below do not contribute. Moreover, the basic assumption of stationarity of the averaged model holds for the frequencies and growth rates obtained.

Thus we have identified an instability by linear analysis of an averaged model, which resembles the shock oscillations observed in the numerical simulations, both with respect to timescales and spatial structure. We therefore conclude, that the shock oscillations are not numerical artifacts. Rather they have a physical origin and are caused by an instability whose mechanism will be investigated in detail in the following sections.

\subsubsection{Approximations}

In order to gain further insight into the physical processes responsible for the instability, different approximations in equations (3.3)-(3.6) have been considered. To obtain a continuous transition from the exact treatment to the approximation, we introduce a parameter $\Phi$ with $\Phi=1$ corresponding to the exact problem and $\Phi \rightarrow 0, \infty$ to the approximation. The numerical results, i.e. the eigenvalues of the shock front instabilities, are followed as a function of $\Phi$.

Introducing $\Phi$ into the Euler equation as

$$
p^{\prime}=-\left(4+\Phi \cdot C_{3} \sigma^{2}\right) \zeta-p
$$

the limit $\Phi \rightarrow 0$ corresponds to vanishing acceleration and implies the elimination of acoustic modes from the spectrum, which then only consists of secular modes. Application of this limit to the shock instabilities has not revealed any unstable modes. Rather the eigenvalues have diverged. This excludes a thermal origin of both the unstable modes and the instability mechanism. For a proper treatment of the instability, the mechanical acceleration has to be taken into account.

Introducing $\Phi$ into the equation for energy conservation as

$$
l^{\prime}=C_{1} \cdot \Phi \cdot(i \sigma)\left(-p+C_{2} t\right)
$$


the adiabatic limit is obtained by $\Phi \rightarrow \infty$. The latter implies $\left(-p+C_{2} t\right)=0$, i.e. the algebraic adiabatic relation between pressure and temperature perturbation. No unstable modes have been found following the shock instabilities into the adiabatic limit.

Introducing $\Phi$ into the equation for energy conservation as

$$
l^{\prime}=C_{1} \cdot \Phi \cdot(i \sigma)\left(-p+C_{2} t\right)
$$

$\Phi \rightarrow 0$ corresponds to the so called NAR-limit (Non-Adiabatic-Reversible limit) (Gautschy \& Glatzel 1990). Although this approximation - like the adiabatic approximation - implies constant entropy, it does not represent the adiabatic limit $\left(-p+C_{2} t\right)=0$. Rather it is equivalent to $C_{1} \rightarrow 0$. Since $C_{1}$ is related to the thermal and dynamical timescales $\tau_{t h}$ and $\tau_{d y n}$ by

$$
C_{1}=\frac{\tau_{t h}}{\tau_{d y n}} \nabla_{a d} \sqrt{\frac{C_{3} C_{4}}{\Gamma}}
$$

this approximation is also being referred to as the zero thermal timescale approximation ( $\Gamma$ and $\nabla_{a d}$ are the adiabatic indices). Physically, it means that the specific heat of the envelope is negligible and luminosity perturbations cannot be sustained. In particular, this approximation rules out the classical $\kappa$-mechanism as the source of an instability should it exist in the NAR-limit - since this Carnot-type process relies on a finite heat capacity. When following the frequencies of the modes belonging to the shock front instabilities into the NAR-limit, periods and growth rates change only slightly (by at most 10 per cent). Thus the NAR-approximation may be regarded as a satisfactory approximation and will form the basis of our investigations in the following sections.

\subsection{An analytical model}

\subsubsection{Three-Zone-Model}

The modal structure identified in section 6.3.2 with three sets of modes associated with three acoustic cavities (inner envelope, shock and outer envelope) suggests the construction of a three zone model. In order to enable an analytical solution, the coefficients of the differential equations are kept constant in each zone.

According to the previous section the NAR-approximation is sufficient to describe the shock front instabilities. The equation of energy conservation is then satisfied identically and luminosity perturbations vanish. Thus we are left with a system of third order comprising the mechanical equations and the diffusion equation with zero luminosity perturbation.

Further reduction of the order of the differential system is achieved by considering its coefficients which depend on the properties of the averaged model. In figure 6.5 the coefficients $C_{5}=\alpha=\left.\frac{\partial \log \rho}{\partial \log p}\right|_{T} \approx \frac{1}{\beta}$ and $C_{7}=\nabla=\frac{\mathrm{d} \log T}{\mathrm{~d} \log p}$ are shown as a function of relative radius. $\beta$ denotes the ratio of gas pressure to total pressure. The coefficients 

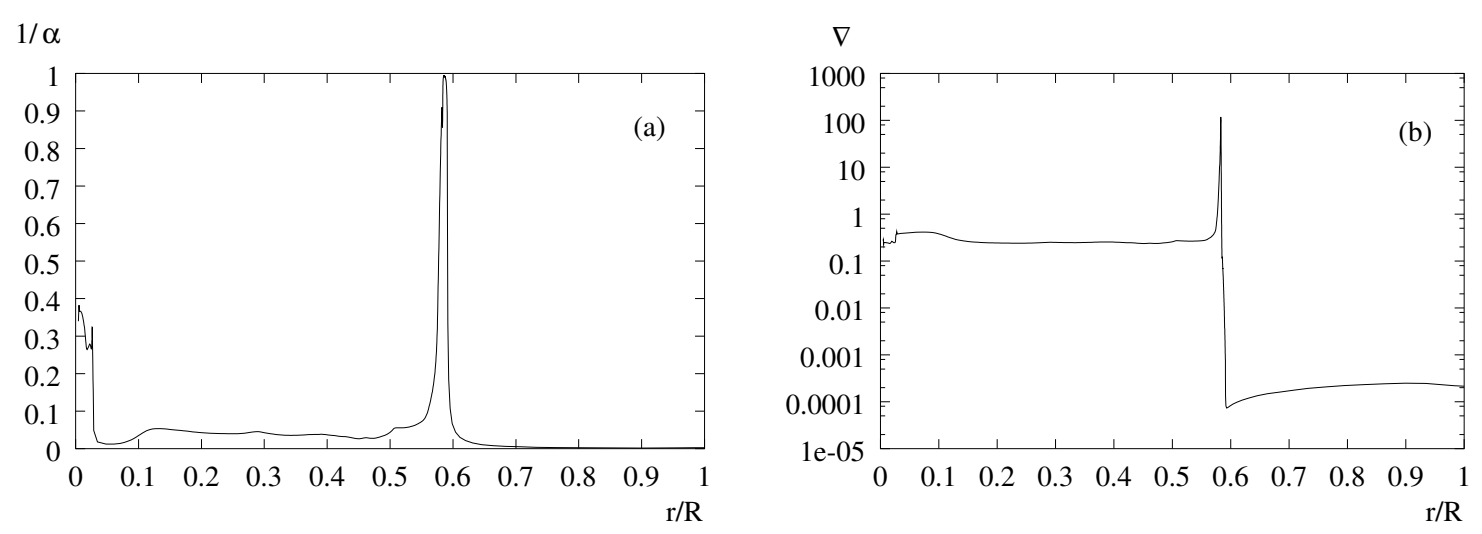

Figure 6.5: The coefficients $C_{5}=\alpha$ (a) and $C_{7}=\nabla$ (b) of the averaged model as a function of relative radius.

$C_{4}=-\frac{d \log r}{d \log p}$ and $C_{3}=\frac{4 \pi r^{3} \bar{\rho}}{M_{r}}$ may be regarded as constant all over the envelope. Approximate values are $C_{4} \approx \frac{1}{3}$ and $C_{3} \approx 3$. The latter holds because almost the entire mass is concentrated in the stellar core. From figure 6.5 we deduce that radiation pressure is dominant except for the shock zone. Therefore we replace the diffusion equation 3.6 by the algebraic equation of state for pure radiation $(p=4 t)$ in the inner and outer envelope. On the other hand $C_{7}=\nabla$ can - to first approximation - be regarded as singular in the shock zone. According to equation 3.6 this requires the expression $\left(-4 \zeta+C_{8} p-C_{9} t\right)$ to vanish there. Thus the differential diffusion equation is replaced by an algebraic relation in all three zones, reducing the system to second order.

Adopting the alternative notation (Baker \& Kippenhahn 1962) $C_{6}=\delta, C_{8}=\kappa_{p}$ and $C_{9}=4-\kappa_{T}$, where $\delta$ is the negative logarithmic derivative of density with respect to temperature at constant pressure, $\kappa_{p}$ the logarithmic derivative of opacity with respect to pressure at constant temperature and $\kappa_{T}$ the logarithmic derivative of opacity with respect to temperature at constant pressure, and choosing the relative radius $x$ as the independent variable, we are left with the following set of equations:

$$
\begin{aligned}
\frac{1}{\psi} \frac{d \zeta}{d x} & =\frac{1}{3}(3 \zeta+\alpha p-\delta t) \\
\frac{1}{\psi} \frac{d p}{d x} & =-\left(4+3 \sigma^{2}\right) \zeta-p \\
t & = \begin{cases}\left(\frac{1}{4-\kappa_{T}}\right)\left(\kappa_{p} p-4 \zeta\right) & x \in[a, b] \\
\frac{1}{4} \cdot p & x \in[0, a) \text { or } x \in(b, 1]\end{cases}
\end{aligned}
$$

$a$ and $b$ denote the lower and upper boundary of the shock zone. The transformation of the independent variables $\ln p_{0} \rightarrow x$ introduces the factor $\psi$, which is constant within the framework of the three-zone-model, and given by an appropriate mean of the quantity $\frac{-1}{C_{4} x}$. In general $\psi$ is negative and of order unity.

We are thus left with a system of second order consisting of the mechanical equations (continuity and Euler equations) which is closed by the algebraic relations $0=-4 \zeta+$ 
$C_{8} p-C_{9} t$ and $p=4 \cdot t$ for the shock region and the inner/outer regions, respectively. We rewrite it as:

$$
\begin{aligned}
& \frac{d \zeta}{d x}=A_{1,2} \cdot \zeta+B_{1,2} \cdot p \\
& \frac{d p}{d x}=C \cdot \zeta+D \cdot p
\end{aligned}
$$

where

$$
\begin{aligned}
& A_{1,2}= \begin{cases}\psi\left(1+\frac{\frac{4 \delta}{3}}{4-\kappa_{T}}\right) & x \in[a, b] \\
\psi & x \in[0, a) \text { or } x \in(b, 1]\end{cases} \\
& B_{1,2}= \begin{cases}\psi\left(\frac{\alpha}{3}-\frac{\frac{\delta \kappa_{p}}{3}}{4-\kappa_{T}}\right) & x \in[a, b] \\
\psi\left(\frac{\alpha}{3}-\frac{\delta}{12}\right) & x \in[0, a) \text { or } x \in(b, 1]\end{cases} \\
& C=-\psi\left(4+3 \sigma^{2}\right) \\
& D=-\psi
\end{aligned}
$$

and the subscript 1 denotes the values of the coefficients in the shock region, the subscript 2 values in the inner and outer regions. We introduce new variables by

$$
\begin{aligned}
& \hat{\zeta}=e^{\int A_{1,2} d x} \cdot \zeta \\
& \hat{p}=e^{\int D d x} \cdot p
\end{aligned}
$$

The system 6.24-6.25 then reads

$$
\begin{aligned}
& \frac{d \hat{\zeta}}{d x}=B_{1,2} \cdot \hat{p} \cdot e^{\int D d x} \cdot e^{-\int A_{1,2} d x} \\
& \frac{d \hat{p}}{d x}=C \cdot \hat{\zeta} \cdot e^{-\int D d x} \cdot e^{\int A_{1,2} d x}
\end{aligned}
$$

These equations are equivalent to the following single second order equation:

$$
\frac{d}{d x}\left(\frac{1}{C} \cdot e^{\int D d x} \cdot e^{-\int A_{1,2} d x} \cdot \frac{d \hat{p}}{d x}\right)-e^{\int D d x} e^{-\int A_{1,2} d x} \cdot B_{1,2} \cdot \hat{p}=0
$$

\section{Mathematical Structure of the Problem}

Equation 6.36 may be written as

$$
\begin{aligned}
& \frac{d}{d x}\left(e^{\int D d x} \cdot e^{-\int A_{1,2} d x} \cdot \frac{d \hat{p}}{d x}\right)+4 \cdot \psi \cdot e^{\int D d x} e^{-\int A_{1,2} d x} \cdot B_{1,2} \cdot \hat{p} \\
& +3 \sigma^{2} \cdot \psi \cdot e^{\int D d x} e^{-\int A_{1,2} d x} \cdot B_{1,2} \cdot \hat{p}=0
\end{aligned}
$$


and has the form

$$
\frac{d}{d x}\left[q(x) \frac{d}{d x} \hat{p}\right]-w(x) \hat{p}+\lambda u(x) \hat{p}=0
$$

with $q(x)>0$ in the integration interval. However, $u(x)$ is positive in the inner and outer regions and negative in the shock region, i.e., $u(x)$ changes sign in the integration interval. (This holds also for $w(x)$.) Therefore, this problem is not of Sturm-Liouville type. On the other hand, if we consider each zone separately with boundary conditions $\hat{p}=0$, equation 6.38 describes a Sturm-Liouville problem. In the shock zone we define eigenvalues $\lambda=-\sigma^{2}$ and thus have $u(x)>0, w(x)>0$, for the inner and outer zones we get $u(x)>0, w(x)<0$ by defining $\lambda=\sigma^{2}$.

For a Sturm-Liouville problem, the eigenvalues are real and form a sequence

$$
\lambda_{1}<\lambda_{2}<\lambda_{3}<\lambda_{4}<\ldots
$$

Furthermore, $\lambda_{1}$ may be estimated on the basis of the variational principle

$$
\lambda_{1}=\min _{\hat{p}} \frac{\int_{0}^{1} q(x)\left|\hat{p}^{\prime}\right|^{2} d x+\int_{0}^{1} w(x)|\hat{p}|^{2} d x}{\int_{0}^{1} u(x)|\hat{p}|^{2} d x}
$$

Therefore $\lambda_{1}=-\sigma_{1}^{2}$ is positive in the shock zone, since $w(x)$ is positive there. This means that we have purely imaginary eigenfrequencies $\sigma_{j}= \pm i \sqrt{\lambda_{j}}$ with positive $\lambda_{j}$ and

$$
\left|\sigma_{1}\right|<\left|\sigma_{2}\right|<\left|\sigma_{3}\right|<\left|\sigma_{4}\right|<\ldots
$$

Thus the shock zone provides unstable eigenfrequencies.

Since $w(x)$ is negative in the inner and outer zones, we cannot guarantee $\lambda_{1}$ to be positive there. For sufficiently large $j$, however, $\lambda_{j}$ will always become positive. As a consequence, all eigenfrequencies $\sigma_{j}= \pm \sqrt{\lambda_{j}}$ will become real for sufficiently high order $j \geq n$ and satisfy:

$$
\left|\sigma_{n}\right|<\left|\sigma_{n+1}\right|<\left|\sigma_{n+2}\right|<\left|\sigma_{n+3}\right|<\ldots
$$

In principle, the mathematical structure of the problem allows for imaginary pairs of eigenfrequencies at low orders in the inner and outer zones. For the particular parameters studied in the following sections, however, $\lambda_{1}$ turned out to be positive, i.e., $n=1$ and all eigenfrequencies are real.

Even if equation 6.37 together with the boundary conditions $\hat{p}=0$ at $x=0$ and $x=1$ (three-zone-model) is not of Sturm-Liouville type, the differential operator

$$
\mathcal{D}=\frac{d}{d x}\left(q \frac{d}{d x}\right)+\lambda u-w
$$

in equation 6.38 can be shown to be self adjoint with the boundary conditions $\hat{p}=0$ at $x=0$ and $x=1$. Therefore the eigenvalues $\lambda$ are real and we do expect only real or purely imaginary eigenfrequencies $\sigma$, i.e., we will not be able to reproduce the complex eigenfrequencies of the exact problem in this approximation. 


\section{Results}

Assuming the coefficients $C$ and $B_{1,2}$ to be constant, equations 6.34 and 6.35 are solved by the Ansatz $\hat{p}, \hat{\zeta} \propto e^{k x}$. For the wavenumbers $k$ we get

$$
k= \pm \sqrt{B_{1,2} \cdot C}
$$

Thus the general solutions reads

$$
\hat{p}= \begin{cases}a_{1} \cdot e^{\sqrt{B_{2} C} x}+a_{2} \cdot e^{-\sqrt{B_{2} C x}} & x \in[0, a) \\ b_{1} \cdot e^{\sqrt{B_{1} C} x}+b_{2} \cdot e^{-\sqrt{B_{1} C x}} & x \in[a, b] \\ c_{1} \cdot e^{\sqrt{B_{2} C x}}+c_{2} \cdot e^{-\sqrt{B_{2} C x}} & x \in(b, 1]\end{cases}
$$

$a_{1,2}, b_{1,2}$ and $c_{1,2}$ are integration constants and have to be determined by the requirements of continuity and differentiability of $\hat{p}$ at $x=a$ and $x=b$ and the boundary conditions at $x=0$ and $x=1$. For the latter we choose $\hat{p}=0$, which implies

$$
\begin{aligned}
& a_{2}=-a_{1} \quad \text { and } \\
& c_{2}=-c_{1} \cdot e^{2 \sqrt{B_{2} C}}
\end{aligned}
$$

Together with the requirements of continuity and differentiability this yields the dispersion relation

$$
\begin{aligned}
& \frac{\left(\sqrt{B_{1} C}-\sqrt{B_{2} C}\right) e^{\sqrt{B_{2} C} a}+\left(-\sqrt{B_{1} C}-\sqrt{B_{2} C}\right) e^{-\sqrt{B_{2} C a}}}{\left(\sqrt{B_{1} C}+\sqrt{B_{2} C}\right) e^{\sqrt{B_{2} C a}+\left(-\sqrt{B_{1} C}+\sqrt{B_{2} C}\right) e^{-\sqrt{B_{2} C a}}} \cdot e^{2 \sqrt{B_{1} C}(a-b)}=} \\
& \frac{\left(\sqrt{B_{1} C}-\sqrt{B_{2} C}\right) e^{\sqrt{B_{2} C}(b-1)}+\left(-\sqrt{B_{1} C}-\sqrt{B_{2} C}\right) e^{-\sqrt{B_{2} C}(b-1)}}{\left(\sqrt{B_{1} C}+\sqrt{B_{2} C}\right) e^{\sqrt{B_{2} C}(b-1)}+\left(-\sqrt{B_{1} C}+\sqrt{B_{2} C}\right) e^{-\sqrt{B_{2} C(b-1)}}}
\end{aligned}
$$

where the eigenfrequencies $\sigma$ are contained in the coefficient $C$. In general, the roots of equation 6.48 have to be calculated numerically, using, for example, a complex secant method. Separate spectra for the three isolated zones may be obtained by assuming the boundary conditions $\hat{p}=0$ at $x=a, b$ instead of continuity and differentiability requirements. We are then left with the dispersion relations

$$
\begin{aligned}
1 & =\frac{\left(\sqrt{B_{1} C}-\sqrt{B_{2} C}\right) e^{\sqrt{B_{2} C} a}+\left(-\sqrt{B_{1} C}-\sqrt{B_{2} C}\right) e^{-\sqrt{B_{2} C} a}}{\left(\sqrt{B_{1} C}+\sqrt{B_{2} C}\right) e^{\sqrt{B_{2} C} a}+\left(-\sqrt{B_{1} C}+\sqrt{B_{2} C}\right) e^{-\sqrt{B_{2} C a}}} \\
1 & =\frac{\left(\sqrt{B_{1} C}+\sqrt{B_{2} C}\right) e^{\sqrt{B_{2} C}(b-1)}+\left(-\sqrt{B_{1} C}+\sqrt{B_{2} C}\right) e^{-\sqrt{B_{2} C}(b-1)}}{\left(\sqrt{B_{1} C}-\sqrt{B_{2} C}\right) e^{\sqrt{B_{2} C}(b-1)}+\left(-\sqrt{B_{1} C}-\sqrt{B_{2} C}\right) e^{-\sqrt{B_{2} C}(b-1)}} \\
1 & =e^{2 \sqrt{B_{1} C}(a-b)}
\end{aligned}
$$

for the inner, outer and shock zones, respectively.

For the averaged model we have $B_{1} \approx-4 \psi$ and dominant radiation pressure implies $B_{2} \approx \frac{1}{4} \psi$. Inserting these values into equations 6.49-6.50 we are left with

$$
\begin{array}{ccc}
\sqrt{\frac{\psi^{2}}{4}\left(4+3 \sigma^{2}\right)} a & =\frac{(2 n+1) \pi}{2} & n \in \mathbb{Z} \\
\sqrt{\frac{\psi^{2}}{4}\left(4+3 \sigma^{2}\right)}(b-1) & =\frac{(2 n+1) \pi}{2} & n \in \mathbb{Z}
\end{array}
$$


Table 6.3: Eigenfrequencies $\sigma$ ( $\sigma_{r}$ : real part, $\sigma_{i}$ : imaginary part) of the three-zone-model having the parameters $B_{1}=-4 \psi, B_{2}=\frac{\psi}{4}, a=0.57, b=0.59, \psi=-1$

\begin{tabular}{rrrrrrrr}
\hline$\sigma_{r}$ & 12.01 & 16.78 & 18.55 & 24.86 & 25.83 & 31.29 & 34.67 \\
$\sigma_{i}$ & 0 & 0 & 0 & 0 & 0 & 0 & 0 \\
\hline$\sigma_{r}$ & 0 & 0 & 0 & 0 & 0 & & \\
$\sigma_{i}$ & 7.21 & 52.43 & 97.77 & 143.11 & 188.46 & & \\
\hline
\end{tabular}

Thus we have real $\sigma$, i.e., neutrally stable modes, if the inner and outer regions are considered separately, in accordance with the discussion in section 6.4.1. For the shock region equation 6.51 yields

$$
\left.2 \sqrt{4 \psi^{2}\left(4+3 \sigma^{2}\right.}\right)(b-a)=2 \pi n i \quad n \in \mathbb{Z}
$$

These solutions correspond to purely imaginary $\sigma$ implying instability. The solutions of equations 6.52-6.54 can be used as initial guesses for the numerical iteration of equation 6.48 , the dispersion relation of the three-zone-model. Some representative eigenvalues of the three-zone-model are given in Table 6.3. by

Once the eigenfrequencies are determined, the corresponding eigenfunctions are given

$$
p= \begin{cases}a_{1} \cdot e^{\psi x} \cdot e^{\sqrt{B_{2} C x}}+a_{2} \cdot e^{\psi x} \cdot e^{-\sqrt{B_{2} C} x} & x \in[0, a) \\ b_{1} \cdot e^{\psi x} \cdot e^{\sqrt{B_{1} C} x}+b_{2} \cdot e^{\psi x} \cdot e^{-\sqrt{B_{1} C x}} & x \in[a, b] \\ c_{1} \cdot e^{\psi x} \cdot e^{\sqrt{B_{2} C x}}+c_{2} \cdot e^{\psi x} \cdot e^{-\sqrt{B_{2} C x}} & x \in(b, 1]\end{cases}
$$

The factor $e^{\psi x}$ is due to the transformation from $p$ to $\hat{p}$.

Typical eigenfunctions are presented in figures 6.6.a1-6.6.a3. Three types of modes may be distinguished belonging to the three zones of the model. Real eigenfrequencies are associated with the inner and outer region. Except for the shock region they are oscillatory and reach their maximum in the respective region. "Shock modes" correspond to unstable and damped modes (purely imaginary pairs of eigenvalues). They oscillate in the shock region and are evanescent elsewhere. We note the correspondence of figures 6.6.a1 and 6.4.a1, 6.6.a2 and 6.4.a2 and 6.6.a3 and 6.4.a3, i.e., the results of the analytical model resemble those of the exact analysis. The influence of the shock position on the modal structure may also be studied within the framework of the three-zone-model. As long as the width of the shock zone and the coefficient $B_{1}$ are not varied, the "shock modes" are not affected. The dependence on the shock position of the neutrally stable "inner" and "outer" modes is shown in figure 6.6.b. Moving the shock position outwards, the frequencies of the inner modes decrease, whereas those of the outer modes increase, according to the variation of the length of the corresponding acoustic cavities. This leads inevitably to multiple crossings between the frequencies of the inner and outer modes, 

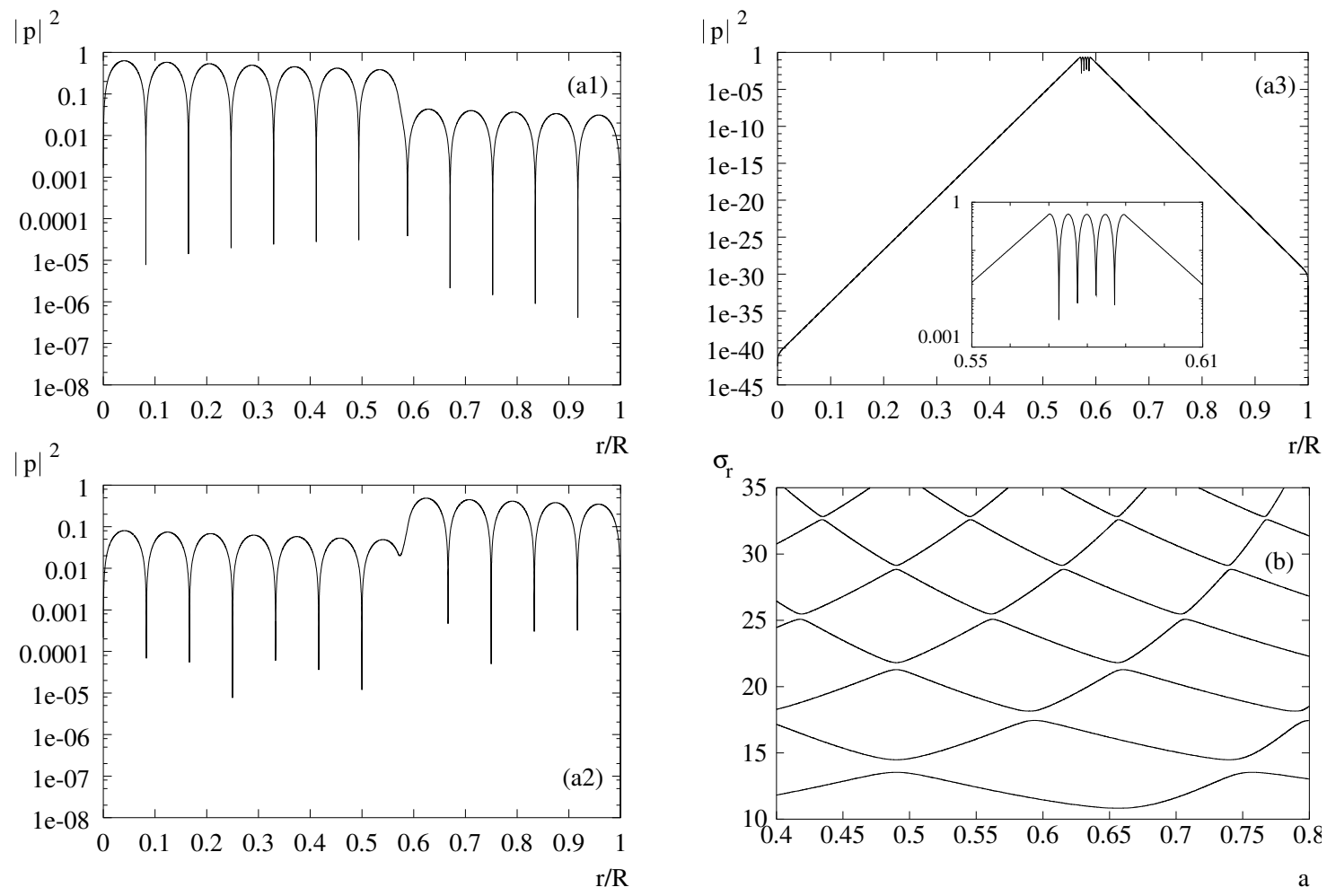

Figure 6.6: Eigenfunctions for the three-zone-model with the parameters $B_{1}=-4 \psi$, $B_{2}=\frac{\psi}{4}, a=0.57, b=0.59, \psi=-1$, and the frequencies $\sigma_{r}=44.04, \sigma_{i}=0$ (a1), $\sigma_{r}=43.53, \sigma_{i}=0$ (a2), $\sigma_{r}=0, \sigma_{i}=188.46$ (a3). (b): Eigenfrequencies $\sigma_{r}$ of neutrally stable modes as a function of the position $a$ of the lower boundary of the shock region for fixed $b-a=0.02$ and $B_{1}=-4 \psi, B_{2}=\frac{\psi}{4}, \psi=-1$.

which unfold into avoided crossings (see, e.g., Gautschy \& Glatzel 1990). Mode interaction by instability bands is excluded here according to the general discussion in section 6.4.1.

\section{Interpretation}

The three-zone-model reproduces the effects of the shock front regarding important aspects: The front acts as an acoustically isolating layer which separates the inner and outer part of the envelope. As a result, these parts provide largely independent spectra. This may be illustrated by the variation of the position of the shock front. Apart from the expected spectra associated with the inner and outer envelope, an additional spectrum of modes is generated by the shock region itself.

Comparing eigenfunctions of the averaged and the analytical model (figures 6.4.a1-a3 and figures 6.6.a1-a3), we find a strikingly similar behaviour. In particular, the confinement of the unstable shock modes is present in both cases. Due to constant coefficients, however, the analytical model reproduces neither decreasing amplitudes nor increasing 
spatial frequencies towards the stellar centre.

We have identified unstable modes in the shock zone of the analytical model. They resemble those of the shock instabilities of the averaged model, and are related to the sound travel time across the shock zone. Its radial extent is primarily responsible for their high frequencies.

The analysis in section 6.4.1 has shown, that the sign of $u(x)$ in equation 6.38 is responsible for the instability in the shock region. This sign is determined by the term $\frac{B_{1}}{\psi}$, which is given by

$$
\frac{B_{1}}{\psi}=\frac{\alpha}{3}-\frac{\frac{\delta \kappa_{p}}{3}}{4-\kappa_{T}}
$$

Estimating the various terms in equation 6.56, we find that the sign of $\kappa_{p}$ determines the sign of $\frac{B_{1}}{\psi}$. A dependence on the sign of $\kappa_{p}$ of the instability, however, is not recovered in the exact problem, which can be tested by replacing $\kappa_{p}$ with $-\kappa_{p}$ there. The exact problem is not affected by this substitution. Thus we conclude, that the analytical model does not provide correct results in this respect and needs to be refined to describe the instability properly. In order to investigate the origin of the instability, some of the simplifying assumptions of the analytical model need to be dropped. In this direction, a more realistic model of the shock zone will be presented in the following section.

\subsubsection{Shock-Zone-Model}

Our study of the three-zone-model in section 6.4.1 has shown, that inner, outer and shock zones may to good approximation be treated separately by assuming suitable boundary conditions, e.g., vanishing pressure at boundaries and interfaces. Moreover, the instabilities of interest are not provided by the inner and outer zones. Therefore we restrict the following study to the shock zone by applying the boundary conditions $p(a)=p(b)=0$. Within the framework of the analytical model the coefficients of the perturbation equations are taken to be constant with the values given in section 6.4.1.

Contrary to section 6.4.1 we will not replace the diffusion equation by an algebraic relation here, as this turned out to lead to erroneous results. However, we still adopt the NAR-approximation. The set of equations considered then reads:

$$
\begin{aligned}
& \frac{1}{\psi} \frac{d \zeta}{d x}=\frac{1}{3}(3 \zeta+\alpha p-\delta t) \\
& \frac{1}{\psi} \frac{d p}{d x}=-\left(4+3 \sigma^{2}\right) \zeta-p \\
& \frac{1}{\psi} \frac{d t}{d x}=\nabla\left(-4 \zeta+\kappa_{p} p-\left(4-\kappa_{T}\right) t\right) \\
& \frac{1}{\psi} \frac{d l}{d x}=0
\end{aligned}
$$


Written in matrix form this yields

$$
\frac{1}{\psi} \frac{d}{d x}\left(\begin{array}{l}
\zeta \\
p \\
t \\
l
\end{array}\right)=\left(\begin{array}{cccc}
1 & \frac{\alpha}{3} & -\frac{\delta}{3} & 0 \\
-\left(4+3 \sigma^{2}\right) & -1 & 0 & 0 \\
-4 \nabla & \nabla \kappa_{p} & -\nabla\left(4-\kappa_{T}\right) & 0 \\
0 & 0 & 0 & 0
\end{array}\right)\left(\begin{array}{l}
\zeta \\
p \\
t \\
l
\end{array}\right)
$$

The differential equation is solved by an exponential dependence $\propto e^{i k x}$ of the dependent variables. Thus we arrive at the linear algebraic equation

$$
\left(\begin{array}{cccc}
1-\frac{i k}{\psi} & \frac{\alpha}{3} & -\frac{\delta}{3} & 0 \\
-\left(4+3 \sigma^{2}\right) & -1-\frac{i k}{\psi} & 0 & 0 \\
-4 \nabla & \nabla \kappa_{p} & -\nabla\left(4-\kappa_{T}\right)-\frac{i k}{\psi} & 0 \\
0 & 0 & 0 & -\frac{i k}{\psi}
\end{array}\right)\left(\begin{array}{l}
\zeta \\
p \\
t \\
l
\end{array}\right)=\left(\begin{array}{l}
0 \\
0 \\
0 \\
0
\end{array}\right)
$$

This equation has a non trivial solution only if the determinant of the matrix vanishes, which provides a quartic equation for the wavenumber $k$. One of its roots is zero, the remaining three roots are determined by the following cubic equation:

$$
\begin{aligned}
& \left(\frac{i k}{\psi}\right)^{3} \frac{1}{\nabla\left(4-\kappa_{T}\right)}+\left(\frac{i k}{\psi}\right)^{2}+ \\
& \left(\frac{i k}{\psi}\right) \underbrace{\left(-\frac{\frac{4}{3} \delta}{\left(4-\kappa_{T}\right)}-\frac{1}{\nabla\left(4-\kappa_{T}\right)}\left(1-\frac{\alpha}{3}\left(4+3 \sigma^{2}\right)\right)\right)}_{d_{1}}+ \\
& \underbrace{\left(-1+\frac{\alpha}{3}\left(4+3 \sigma^{2}\right)-\frac{1}{\left(4-\kappa_{T}\right)}\left(\frac{\delta}{3} \kappa_{p}\left(4+3 \sigma^{2}\right)+\frac{4}{3} \delta\right)\right)}_{d_{2}}=0
\end{aligned}
$$

In the limit of large $\nabla$ they may be given in closed form:

$$
\begin{aligned}
& \left(\frac{i k}{\psi}\right)_{1,2}=-\frac{d_{1}}{2} \pm \sqrt{\frac{d_{1}^{2}}{4}-d_{2}} \\
& \left(\frac{i k}{\psi}\right)_{3}=-\nabla\left(4-\kappa_{T}\right)
\end{aligned}
$$

The general solution to the perturbation problem consists of a superposition of four fundamental solutions associated with the four roots for the wavenumber, two of which are oscillatory (those associated with $k_{1}$ and $k_{2}$ ). The dispersion relation is then derived by imposing four conditions. In addition to the boundary conditions $p=0$ at $x=a, b$, we require the two non oscillatory fundamental solutions not to contribute to the eigensolution. The latter is then only determined by $k_{1}$ and $k_{2}$ :

$$
p=h_{1} \cdot e^{i k_{1} x}+h_{2} \cdot e^{i k_{2} x}
$$


where $h_{1}$ and $h_{2}$ are integration constants. They are determined by the boundary conditions $p=0$ at $x=a, b$, which imply

$$
k_{1}-k_{2}=\frac{2 \pi n}{(a-b)}
$$

where $n \in \mathbb{Z}$ denotes the order of the overtone. Using equation 6.64 we get

$$
\frac{d_{1}^{2}}{4}-d_{2}=-\frac{\pi^{2} n^{2}}{\psi^{2}(a-b)^{2}}
$$

With the definitions of $d_{1}$ and $d_{2}$ (equation 6.63) we arrive at a quadratic equation in $\sigma^{2}$. Expanding the coefficients of $\sigma^{2}$ in terms of $\frac{1}{\nabla}$ and assuming $\frac{\pi^{2} n^{2}}{(a-b)^{2}}$ to be large, we obtain to lowest order in $\frac{1}{\nabla}$ :

$$
\sigma^{4} \frac{\frac{9}{6} \alpha^{2}}{\nabla^{2}\left(4-\kappa_{T}\right)^{2}}-\sigma^{2}\left(\alpha-\frac{\delta \kappa_{p}}{\left(4-\kappa_{T}\right)}\right)+\frac{\pi^{2} n^{2}}{\psi^{2}(a-b)^{2}}=0
$$

Defining

$$
\tilde{\nabla}=\frac{\nabla^{2}\left(4-\kappa_{T}\right)^{2}}{\frac{9}{6} \alpha^{2}}
$$

this equation has the solutions

$$
\begin{aligned}
\sigma_{1,2}^{2}= & \frac{\tilde{\nabla}}{2}\left(\alpha-\frac{\delta \kappa_{p}}{\left(4-\kappa_{T}\right)}\right) \pm \\
& \frac{\tilde{\nabla}}{2}\left(\alpha-\frac{\delta \kappa_{p}}{\left(4-\kappa_{T}\right)}\right) \sqrt{1-\frac{4}{\tilde{\nabla}} \frac{\pi^{2} n^{2}}{\psi^{2}(a-b)^{2}} \frac{1}{\left(\alpha-\frac{\delta \kappa_{p}}{\left(4-\kappa_{T}\right)}\right)^{2}}}
\end{aligned}
$$

In the NAR-approximation, eigenfrequencies come in complex conjugate pairs, i.e., complex eigenfrequencies imply instability. According to equation 6.71, complex eigenfrequencies, and therefore instability, are obtained, if $\nabla$ is finite and $n$ is sufficiently large. For fixed $n$ we obtain in the limit of large $\nabla$ (expansion of the root):

$$
\begin{aligned}
\sigma_{1}^{2} & =\frac{1}{4} \frac{\pi^{2} n^{2}}{\psi^{2}(a-b)^{2}} \frac{1}{\left(\alpha-\frac{\delta \kappa_{p}}{\left(4-\kappa_{T}\right)}\right)} \\
\sigma_{2}^{2} & =\tilde{\nabla}\left(\alpha-\frac{\delta \kappa_{p}}{\left(4-\kappa_{T}\right)}\right)-\frac{2 \pi^{2} n^{2}}{\psi^{2}(a-b)^{2}} \frac{1}{\left(\alpha-\frac{\delta \kappa_{p}}{\left(4-\kappa_{T}\right)}\right)}
\end{aligned}
$$

Equation 6.72 describes the eigenfrequencies of the decoupled shock modes discussed in section 6.4.1, i.e., the second order analysis of the previous section is contained in the limit $\nabla \rightarrow \infty$ of the present approach. Instabilities described by equation 6.71 resemble those of the averaged model, rather than those given by equation 6.72 for positive values of $\kappa_{p}$. We conclude that a finite but large value of the stratification parameter $\nabla=$ $\frac{\mathrm{d} \log T}{\mathrm{~d} \log p}$ is essential for instability. However, assuming $\nabla \rightarrow \infty$, which was done in the investigation of the three-zone-model (section 6.4.1), is an oversimplification. 


\subsection{Conclusions}

When following the non linear evolution of strange mode instabilities in the envelopes of massive stars, shock fronts were observed to be captured in the H-ionisation zone some pulsation periods after reaching the non linear regime. This effect is not observed in models of very hot envelopes (such as the massive star model investigated by Glatzel et al. (1999)), due to hydrogen being ionised completely. The shocks trapped in the $\mathrm{H}$-ionisation zone perform high frequency oscillations (associated with the sound travel times across the shock zone) confined to its very vicinity, whereas the remaining parts of the envelope vary on the dynamical timescale of the primary, strange mode instability. By performing an appropriate linear stability analysis the high frequency oscillations were shown to be due to a physical instability, rather than being a numerical artifact.

An analytical model for the secondary, shock zone instabilities has been constructed. As a result, high values of $\nabla$ were found to be responsible for instability. Contrary to the common stratification (convective, Rayleigh-Taylor) instabilities driven by buoyancy forces and thus associated with (non radial) gravity modes, however, the instabilities found here are associated with spherically symmetric acoustic waves. An extension of the stability analysis to non radial perturbations would be instructive, since we expect the acoustic instabilities identified here - similar to strange mode instabilities (see Glatzel \& Mehren 1996) - not to be restricted to spherical geometry. Such an investigation would also reveal buoyancy driven instabilities, which we believe not to be relevant for the following reasons: Their typical timescale is much longer than that of the acoustic instabilities, which will therefore dominate the dynamics. Moreover, in addition to gravity, the acceleration due to the shocks velocity field has to be taken into account and is likely to stabilise the stratification with respect to convective instabilities. With respect to the aim of this paper to identify the secondary, shock oscillations and their origin, a non radial analysis is beyond the scope of the present investigation and will be the subject of a forthcoming publication.

Since the oscillations are a physical phenomenon - rather than a numerical artifact they should not be damped by increasing the artificial viscosity as one would neglect a physical process whose influence on the long term behaviour of the system cannot yet be predicted. On the other hand, following the shock oscillations by numerical simulation for more than a few dynamical timescales is not feasible due to the small timesteps necessary to resolve them. The confinement of the oscillations to the very vicinity of hydrogen ionisation, however, indicates a solution of the problem by means of domain decomposition: The stellar envelope is decomposed into three domains: below, around and above the shock. Only the narrow shock region needs high time resolution, the inner and outer zones merely require the dynamical timescale to be resolved. The development of a code following this strategy is in progress.

Even if the appearance of the shock oscillations has so far prevented us from performing simulations in excess of several dynamical timescales, the velocity amplitudes reach a significant fraction of the escape velocity. This indicates that pulsationally driven mass loss may be found in appropriate simulations. Whether the new code will allow for the 
corresponding long term simulations and thus possibly for the determination of mass loss rates, remains to be seen. Preliminary results will be published in a forthcoming paper.

\section{Acknowledgements}

We thank Professor K.J. Fricke for encouragement and support. Financial support by the Graduiertenkolleg "Strömungsinstabilitäten und Turbulenz" (MG) and by the DFG under grant WA 633 12-1 (SC) is gratefully acknowledged. The numerical computations have been carried out using the facilities of the GWDG at Göttingen. 


\section{Bibliography}

Baker N.H., Kippenhahn R., 1962, Z. Astrophysik, 54, 114

Buchler J.R., Whalen P., 1990, in The Numerical Modelling of Nonlinear Stellar

Pulsations Problems and Prospects, ed. J. R. Buchler, NATO ASI ser. C302, Kluver Academic Publishers, Dordrecht, 315

Christy R.F., 1966, in Stein R.F., Cameron A.G.W., ed., Stellar Evolution. Plenum

Press, New York, p 359

Cox J.P., 1980, Theory of Stellar Pulsation. Princeton Univ. Press, Princeton, NJ

Dorfi E. A., Gautschy A., 2000, ApJ, 545, 982

Fraley, G.S., 1968, Ap\&SS, 2, 96

Gautschy A., Glatzel W., 1990, MNRAS, 245, 154

Glatzel W., 1994, MNRAS, 271, 66

Glatzel W., Kiriakidis M. 1993, MNRAS, 263, 375

Glatzel W., Kiriakidis M., Chernigovskij S., Fricke K.J. 1999, MNRAS, 303, 116

Glatzel W., Mehren S. 1996, MNRAS, 282, 1470

Humphreys R.M., Davidson K., 1979, ApJ, 232, 409

Iglesias C.A., Rogers F.J., Wilson B.G., 1992, ApJ, 397, 717

Kiriakidis M., Fricke K.J., Glatzel W. 1993, MNRAS, 264, 50

Rogers F.J., Iglesias C.A., 1992, ApJS, 79, 507

Samarskii A., Popov Yu., 1969, Zh. Vychisl. Mat. i Mat. Fiz., 9, 953

Tscharnuter W. M., Winkler K.-H., 1979, Comp. Phys. Comm., 18, 171

Unno W. Osaki Y., Ando H., Saio H., Shibahashi H., 1989, Nonradial Pulsations. Univ.

Tokyo Press, Tokyo 


\title{
7. Domain decomposition
}

\section{Simulation of stellar instabilities with vastly different time- scales using domain decomposition ${ }^{1}$}

\author{
M. Grott ${ }^{2}$, S. Chernigovski ${ }^{3}$ and W. Glatzel ${ }^{2}$ \\ ${ }^{2}$ Universitäts-Sternwarte Göttingen, Geismarlandstr .11, 37083 Göttingen, Germany \\ ${ }^{3}$ Institut für Analysis und Numerik, Universität Magdeburg, Universitätsplatz 2, 39106 Magdeburg, Germany
}

\begin{abstract}
Strange mode instabilities in the envelopes of massive stars lead to shock waves, which can oscillate on a much shorter timescale than that associated with the primary instability. The phenomenon is studied by direct numerical simulation using a, with respect to time, implicit Lagrangian scheme, which allows for the variation by several orders of magnitude of the dependent variables. The timestep for the simulation of the system is reduced appreciably by the shock oscillations and prevents its long term study. A procedure based on domain decomposition is proposed to surmount the difficulty of vastly different timescales in various regions of the stellar envelope and thus to enable the desired long term simulations. Criteria for domain decomposition are derived and the proper treatment of the resulting inner boundaries is discussed. Tests of the approach are presented and its viability is demonstrated by application to a model for the star P Cygni. In this investigation primarily the feasibility of domain decomposition for the problem considered is studied. We intend to use the results as the basis of an extension to two dimensional simulations.
\end{abstract}

Key words: hydrodynamics - instabilities - shock waves - stars: oscillations - stars: variables: other - stars: individual: P Cygni.

\footnotetext{
${ }^{1}$ This paper has been accepted for publication by MNRAS (Grott et al. 2003b)
} 


\subsection{Introduction}

Sufficiently luminous objects, such as massive stars, are known to suffer from strange mode instabilities with growth rates in the dynamical range (Kiriakidis, Fricke \& Glatzel 1993, Glatzel \& Kiriakidis 1993). The boundary of the domain in the HertzsprungRussel diagram (HRD) above which all stellar models are unstable - irrespective of their metallicity -, coincides with the observed Humphreys-Davidson (HD) limit (Humphreys \& Davidson 1979). Moreover, the range of unstable models covers the stellar parameters for which the LBV (luminous blue variable) phenomenon is observed (for a review see Humphreys \& Davidson 1994).

The high growth rates of the instabilities indicate a connection to the observed mass loss of the corresponding objects. To verify this suggestion, simulations of their evolution into the non linear regime have been performed. In fact, for selected models Glatzel et al. (1999) found the velocity amplitude to exceed the escape velocity (see, however, Dorfi \& Gautschy (2000)).

To identify a possible connection between non linear pulsations and outbursts in luminous blue variables Grott, Glatzel \& Chernigovski (2003) have studied the evolution of an initial model located in the HRD well above the HD limit. In this study, the shocks formed in the non linear regime are captured by the H-ionisation zone after a few pulsation periods. These captured shocks start to oscillate rapidly with periods of the order of the sound travel time across the $\mathrm{H}$-ionisation zone, while its mean position changes on the dynamical timescale of the primary, strange mode instability. Grott et al. (2003) have shown, that this shock front oscillation is of physical origin and therefore must not be disregarded. In particular, the phenomenon should not be eliminated by increasing the artificial viscosity. We note that the representation of the phenomenon requires the correct treatment of extreme gradients of the dependent variables, implying their variation by several orders of magnitude. It is achieved by use of a, with respect to time implicit Lagrangian scheme.

The rapid shock oscillations, which are confined to a narrow region in the vicinity of the shock front, require an inhibitively small timestep and thus prevent long term simulations. In the present paper we propose an approach based on domain decomposition to surmount the difficulty of vastly different timescales in various regions of the stellar envelope and thus to enable the desired long term simulations. In this procedure the various domains within the envelope are to be treated separately and according to their intrinsic timescales. We expect this decomposition to speed up the calculations considerably. An even higher speedup will be achieved when applying domain decomposition to two dimensional simulations. In this sense, the present investigation may be regarded as a preliminary study for decomposition in two dimensions.

The basic equations and assumptions are introduced in Section 7.2. The domain decomposition approach is discussed in detail in Section 7.3, including a derivation of criteria for domain decomposition and an investigation of the proper treatment of the resulting inner boundaries. Moreover, tests of the approach are presented there. Its viability is demonstrated by application to a model of the star P Cygni in section 7.4. Our 
conclusions follow.

\subsection{Basic equations and assumptions}

The evolution of instabilities of a stellar envelope is followed into the non-linear regime assuming spherical symmetry and adopting a Lagrangian description, i.e. the independent variables are the time $\mathrm{t}$ and the mass $M_{r}$ inside a sphere of radius $r$. The equations to be solved (see, e.g., Cox 1980) are given by mass conservation,

$$
\frac{\partial r^{3}}{\partial M_{r}}-\frac{3}{4 \pi \rho}=0
$$

momentum conservation,

$$
\frac{\partial^{2} r}{\partial t^{2}}+4 \pi r^{2} \frac{\partial p}{\partial M_{r}}+\frac{G M_{r}}{r^{2}}=0
$$

energy conservation,

$$
\frac{\partial L}{\partial M_{r}}-\epsilon-\frac{p}{\rho^{2}} \frac{\partial \rho}{\partial t}+\frac{\partial E}{\partial t}=0
$$

and the diffusion equation for energy transport,

$$
\frac{\partial T}{\partial M_{r}}-\frac{3 \kappa\left(L-L_{c o n v}\right)}{64 \pi^{2} a c r^{4} T^{3}}=0
$$

where $\rho, p, T, L$, and $E$ denote density, pressure, temperature, luminosity and specific internal energy, respectively. $a$ is the Stefan-Boltzmann constant, $G$ the gravitational constant and $c$ the speed of light. We emphasise that $\frac{\partial}{\partial t}$ is the substantial time derivative. For the opacities $\kappa$, the latest versions of the OPAL tables (Iglesias, Rogers \& Wilson 1992, Rogers \& Iglesias 1992) have been used. Convection is treated in the standard frozen in approximation (see Baker \& Kippenhahn 1965), i.e. the convective luminosity $L_{c o n v}$ is kept constant and equal to the luminosity of the hydrostatic initial model. Since the instabilities are localised in the outer envelope, the evolution of the core can be neglected. Its properties are taken into account by imposing time independent boundary conditions (e.g. by prescribing luminosity, [vanishing] velocity $v$ and [constant] radius) at the bottom of the envelope. For the envelope model, the nuclear energy generation rate $\epsilon$ vanishes. At the outer boundary, the gradient of heat sources is required to vanish ( $F$ is the heat flux):

$$
\operatorname{grad}(\operatorname{div} F)=0
$$

This boundary condition implies (by using equations 7.1,7.3 and 7.4) boundary values for the temperature $T$ and pressure $p$. It is chosen to ensure that outgoing shocks pass through the boundary without reflection. The set of boundary conditions prescribing 

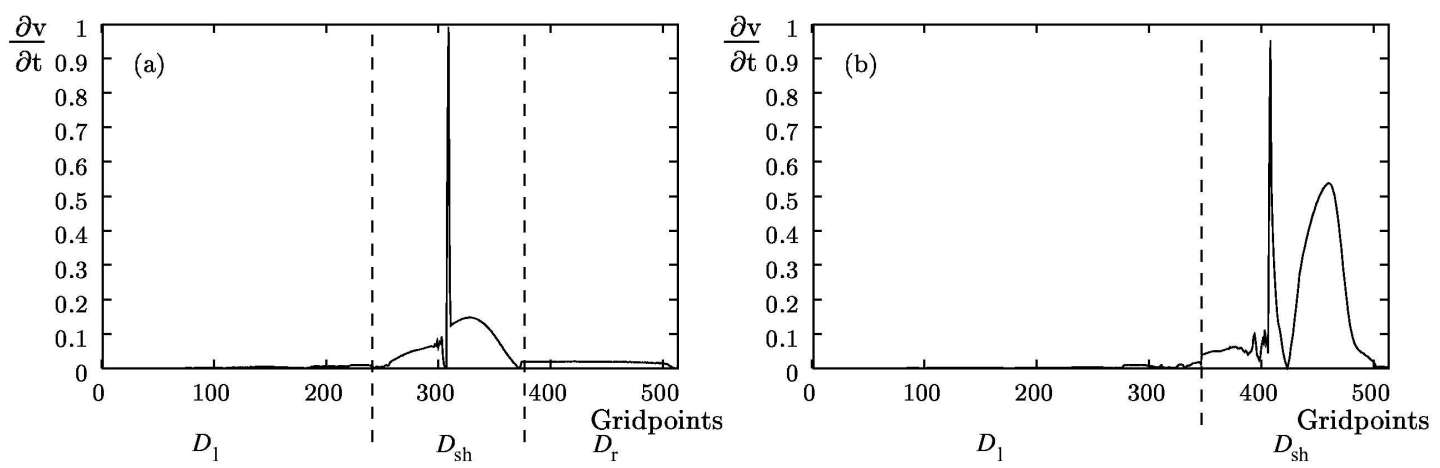

Figure 7.1: Normalised time derivatives of the velocity $v$ as a function of gridpoint, for two typical states of the system. Decomposition into three (a) and two (b) subdomains $\left(\mathcal{D}_{l}, \mathcal{D}_{s h}, \mathcal{D}_{r}\right)$ as indicated by the dashed lines is suggested.

values for the velocity and luminosity at the bottom and values for the pressure and temperature at the top of the envelope will be denoted by $(v, L)(p, T)$ in the following.

The numerical code relies on a Lagrangian, with respect to time implicit, fully conservative difference scheme proposed by Fraley (1968) and Samarskii \& Popov (1969). As a consistent extension the two dimensional version of the code currently under development (also implicit and Lagrangian) is based on the method of support operators originally suggested by Samarskii et al. (1981) and Ardelyan \& Gushchin (1982). For a thorough description of the method of support operators we refer to Shashkov (1996). To handle shock waves, artificial viscosity is used. Concerning tests of the code, we adopted the same criteria as Glatzel et al. (1999). We emphasise, that conservativity of the numerical scheme is of fundamental importance when simulating instabilities in a stellar envelope. Considering the distribution of internal, gravitational and kinetic energy we find, that the kinetic energy can be smaller than, e.g., the gravitational energy by several orders of magnitude. Appropriate simulations of stellar instabilities require the correct representation of the kinetic energy, and therefore energy conservation with high accuracy is indispensable. The difference scheme adopted here guarantees energy conservation.

\subsection{Domain decomposition}

\subsubsection{Motivation for domain decomposition}

The stellar envelope model representing a massive star with mass $M=50 M_{\odot}$, effective temperature $T_{\text {eff }}=10^{4} \mathrm{~K}$ and luminosity $L=1.17 \cdot 10^{6} L_{\odot}$, which was considered by Grott et al. (2003), suffers from strange-mode-instabilities. These cause pulsations with velocity amplitudes of $0.5 v_{\text {esc }}$ and inflate the envelope to 2.5 initial radii. After several pulsation periods a shock front is captured in the H-ionisation zone. The latter 
is prone to secondary instabilities and oscillates on very short timescales connected to the sound travel time across the front. These instabilities are caused by the stratification, but not driven by buoyancy (Grott, Glatzel \& Chernigovski 2003). Resolving the rapid oscillations of the shock front reduces the timestep to very small values. This is computationally extremely expensive and effectively inhibits the desired long term study of the system.

In order to enable the treatment of the problem the integration interval is decomposed such that the small and quickly varying shock region is integrated with small time steps and the remaining major part of the envelope is calculated using large time steps. This strategy of domain decomposition is common in computational fluid dynamics (see, e.g., Wu 1999 and Wu \& Zou 2000). For the treatment of interfaces between the various domains and the associated inner boundary conditions for purely hyperbolic systems, we refer the reader to these publications. We stress, however, some fundamental differences to the previous studies: One of them concerns the different character of the system of equations. So far, only purely hyperbolic systems have been considered, whereas we apply domain decomposition to a composite system of hyperbolic and parabolic equations. Moreover, we adopt a numerical scheme which is implicit in time. Again, this is in contrast to previous studies which use explicit schemes.

\subsubsection{Criterion for domain decomposition}

In this section a criterion for the proper choice of the boundaries of the various domains evolving on different timescales and therefore treated with different time steps will be presented. Considering the time derivatives of various physical quantities the velocity $v$ is found to vary most rapidly and therefore determines the time step.

Figure 7.1 shows the time derivatives of the velocity for two typical states of the system, where Figure 7.1.a corresponds to rapid shock oscillations around gridpoint 310. In this case, domain decomposition as indicated by the dashed lines is suggested. Figure 7.1.b represents a situation, in which the outer envelope is collapsing onto the shock (at gridpoint 410). Rapid variations are now also found above the shock. Accordingly, decomposition into two domains (as indicated by the dashed line) seems appropriate. Depending on the state of the system, we therefore need to split the domain of integration into two or three subdomains.

The size of the various domains is determined by comparing the time derivatives of velocity and temperature with the corresponding derivatives on the shock front. Therefore, as a first step, the position of the shock front has to be determined. For the models studied, the latter is defined by the maximum temperature gradient. The boundaries of the shock zone are then defined by the requirement, that the time derivative at their position corresponds to a given fraction $1 / k$ of the time derivative at the shock. In other words, the set of gridpoints $\mathcal{D}_{s h}$ belonging to the shock zone may be characterised by

$$
\mathcal{D}_{s h}=\left\{n:\left|\frac{\partial v}{\partial t}\right|_{n}>\frac{1}{k}\left|\frac{\partial v}{\partial t}\right|_{s h} \wedge\left|\frac{\partial T}{\partial t}\right|_{n}>\frac{1}{k}\left|\frac{\partial T}{\partial t}\right|_{s h}\right\}
$$


where $n$ denotes the number of the gridpoint and derivatives with index $s h$ correspond to their maximum values found in the shock region. Accordingly, the zones below and above the shock are determined by

$$
\begin{aligned}
\mathcal{D}_{l} & =\left\{n: n \leq \min \left(\mathcal{D}_{s h}\right)\right\} \\
\mathcal{D}_{r} & =\left\{n: n \geq \max \left(\mathcal{D}_{s h}\right)\right\}
\end{aligned}
$$

The parameter $k$ corresponds to the ratio of timesteps in $\mathcal{D}_{l, r}$ and $\mathcal{D}_{s h}$, which favours large values of $k$. On the other hand, the size of $\mathcal{D}_{s h}$ increases with $k$, suggesting smaller values. Therefore optimum results will be obtained for a mean choice. For the considered model a value of $k=15$ turned out to be satisfactory. If the size of the domains $\mathcal{D}_{l}$ or $\mathcal{D}_{r}$ drops below a given value, they are considered to be part of the shock zone, and we arrive at a decomposition into two subdomains. For the model considered the whole grid consists of 512 gridpoints and the zones have a minimum size of 64 gridpoints.

After decomposition, the quickly varying region $\mathcal{D}_{s h}$ is integrated first with timesteps $\tau_{1}, \tau_{2}, \ldots, \tau_{n}$. Then the domains $\mathcal{D}_{l}$ and/or $\mathcal{D}_{r}$ are integrated with the timestep $\tau=$ $\tau_{1}+\tau_{2}+\cdots+\tau_{n}$. The decomposition implies artificial boundaries and boundary conditions between the domains $\mathcal{D}_{l}$ and $\mathcal{D}_{s h}$ and $\mathcal{D}_{r}$ and $\mathcal{D}_{s h}$, respectively. An inconsistency is introduced, if in a first approach the explicit inner boundary conditions are either kept fixed, or linearly extrapolated in time. Both cases are contained in the following extrapolation prescription:

$$
Y_{\text {bound }}=\alpha_{Y}\left(\frac{\left(Y-Y_{\text {old }}\right)}{\tau_{\text {old }}} \cdot \tau^{\prime}+Y\right)+\left(1-\alpha_{Y}\right) \cdot Y
$$

$Y$ stands for $p, T, v, L$, and $\alpha_{Y} \in[0,1]$ is a free extrapolation parameter, which may be chosen independently for each variable. $Y$ refers to the current, $Y_{\text {old }}$ to the previous value of the variable. $\tau_{\text {old }}$ is the last, $\tau^{\prime}$ the current timestep. How the inconsistency may be treated will be discussed in the following sections.

Once the integration of all domains has been performed, one subsequent timestep is done without decomposition. We shall refer to it as the relaxation timestep. Relaxation prevents accumulation of residual errors.

\subsubsection{The iteration procedure}

A mathematically consistent way of integrating the different domains, which solves the problem of artificial fixed boundary conditions is the following iterative procedure:

1. The computation is started at time $t$ and $D_{l}, D_{s h}$ and $D_{r}$ are integrated with fixed boundary values $(v, L)_{t}$ and $(p, T)_{t}$ for each domain. The subscript $t$ denotes values at time $t$.

2. New boundary values $(v, L)_{t+\tau}$ and $(p, T)_{t+\tau}$ are obtained by integration of the adjacent domain. With these boundary values the integration 1 is repeated. 

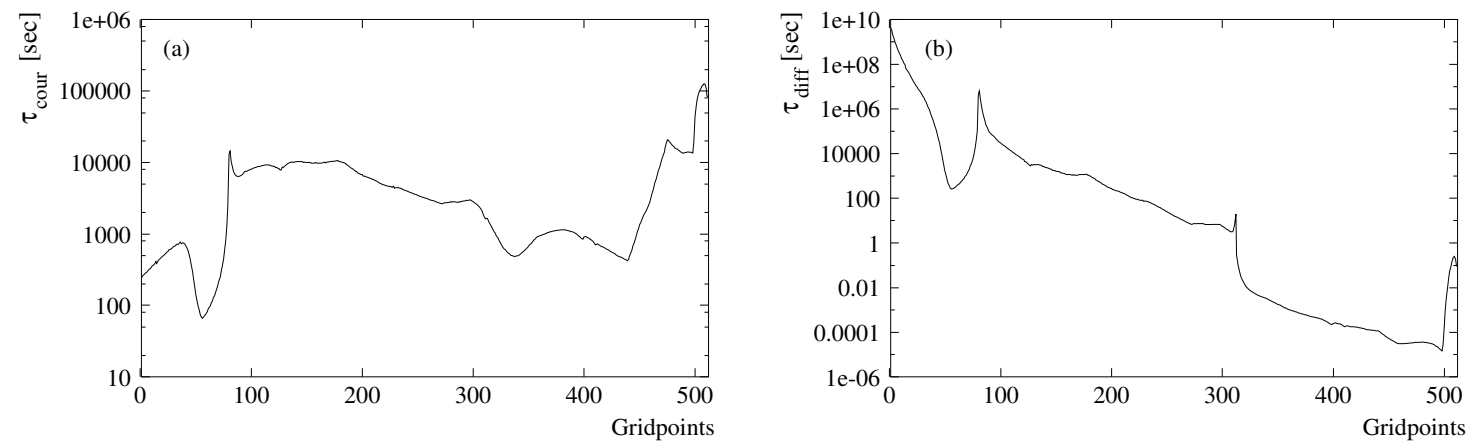

Figure 7.2: Snapshot of the sound travel time $\tau_{\text {cour }}$ (a) and the diffusion time $\tau_{\text {diff }}$ (b) across a cell as a function of gridpoint.

This iterative procedure implies implicit boundary conditions and successively eliminates the errors introduced by the artificial boundaries. 4-5 iteration cycles produce satisfactory results. However, this approach is computationally even more expensive than integrating the entire domain with small timesteps $\tau_{1}, \ldots, \tau_{n}$. Concerning the present problem, it is therefore not relevant and has been applied only for comparison with other methods discussed below. However, for the corresponding two dimensional problem the computational effort might be significantly reduced by the procedure as the inversion of the most ill-conditioned matrix occurring there is an $N^{2}$-process.

\subsubsection{Overlapping domains}

One method to reduce the error caused by the fixed inner boundary conditions consists of using overlapping domains of computation. We emphasise that the system considered here is composed of hyperbolic and parabolic differential equations. We first consider the hyperbolic part, i.e., the mechanical equations. Any errors or perturbations in this part produced at the boundaries of the domains propagate with finite speed into the domain of computation along the characteristics of the equation. For our set of equations, perturbations propagate with velocities $v \pm c_{s}$, where $c_{s}$ denotes the speed of sound. If the domains overlap such that the time for error propagation across the overlap is larger than the integration timestep $\tau$, we may discard the flawed values close to the boundary and keep only the correct values for the subsequent timestep. This condition implies

$$
\tau<\sum_{i} \frac{l_{i}}{v_{i}+c_{s_{i}}}
$$

where the sum of individual sound travel times is to be taken over all overlapping cells. $l_{i}$ denotes the thickness of cell $i$.

Concerning the parabolic part of equations 7.1-7.4, i.e. the diffusion equation for energy transport, perturbations travel across the grid with infinite speed. Therefore, the procedure suggested for the hyperbolic part of the equations can in principle not be carried over to the parabolic part. Rather physical quantities change on the diffusion 
timescale, which is given by $\tau_{\text {diff }}=l^{2} \rho c_{p} \frac{3 \kappa \rho}{4 a c T^{3}}\left(c_{p}\right.$ is the specific heat at constant pressure). Consequently, we expect the effect of the perturbations to be small far from the boundary if the timestep $\tau$ is sufficiently small, i.e. :

$$
\tau<\sum_{i} l_{i}^{2} \rho_{i} c_{p_{i}} \frac{3 \kappa_{i} \rho_{i}}{4 a c T_{i}^{3}}
$$

where the sum of individual diffusion times again extends over all overlapping cells.

In Figure 7.2 a snapshot of the sound travel time (Figure 7.2.a) and diffusion time (Figure 7.2.b) across a cell is given as a function of gridpoint. The sound travel time across the overlapping region is larger than a typical timestep (approximately 1 per cent of the global free fall time) for an overlap of $\sim 8$ cells (condition 7.10). However, while the diffusion time across the overlapping region is bigger than the timestep even for a small overlap in the bottom part of the envelope, condition 7.11 cannot be satisfied in the outer envelope for a reasonable number of overlapping cells. The latter is due to the small heat capacity there (implying the ratio of thermal and dynamical timescales to be small) and in accordance with the validity of the non-adiabatic-reversible approximation (NAR-approximation), which has been shown for this particular stellar model by Grott et al. (2003). How satisfactory results may be obtained even if condition 7.11 is not satisfied will be discussed in the following section.

\subsubsection{Inner boundary conditions}

On the basis of the domain decomposition procedure described above, various inner boundary conditions and their consequences will be investigated in this section. In any case, overlapping domains have been used. The tests presented here have been performed at various times with similar results. Therefore the results may be regarded to be independent of the particular state of the system. In the tests, the luminosity $L$ turned out to be the most sensitive quantity concerning the errors introduced by the inner boundaries. This is due to the fact, that in our difference scheme it is only accurate to first order. Therefore $L$ is required to be reproduced satisfactorily compared to the results of the approach without decomposition.

In Figure 7.3 the luminosity is given as a function of gridpoint around the boundary between $\mathcal{D}_{l}$ and $\mathcal{D}_{s h}$, which is more sensitive than the boundary between $\mathcal{D}_{s h}$ and $\mathcal{D}_{r}$ with respect to the decomposition procedure. For comparison, results obtained without decomposition are shown as solid lines. Dotted lines correspond to results with decomposition, where the left and right columns illustrate those before and after relaxation, respectively.

1. Figure 7.3.a shows the result obtained using the $(v, L)(p, T)$ boundary condition, i.e., velocity and luminosity were prescribed at the left, and temperature and pressure at the right boundary, respectively. This condition implies a discontinuity of the luminosity (a1) and leads to an unacceptable relative error $(\sim$ 15 per cent) after relaxation (a2). 

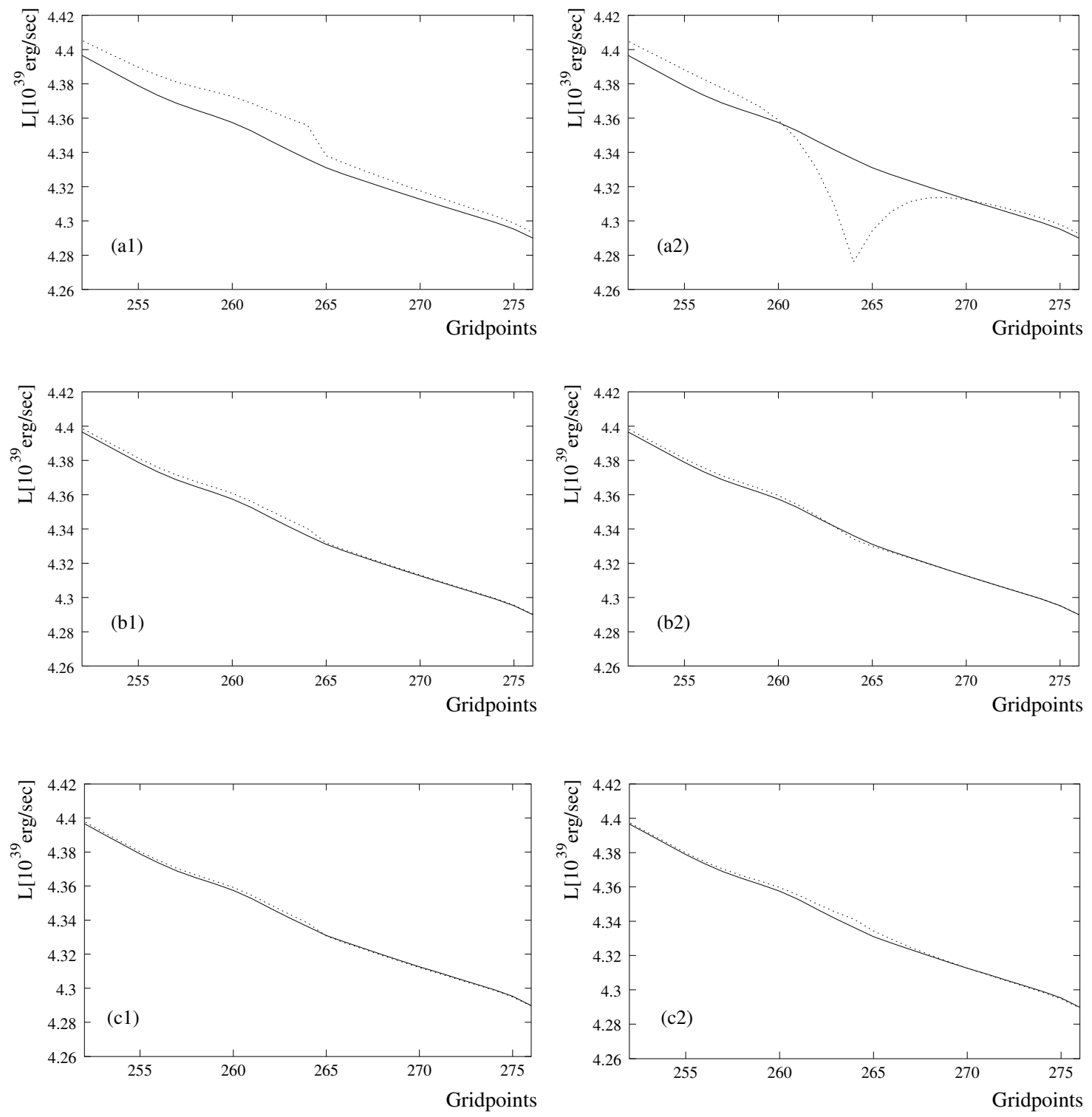

Figure 7.3: The luminosity as a function of gridpoint around the boundary between $\mathcal{D}_{l}$ and $\mathcal{D}_{s h}$ at gridpoint 264. Results obtained without decomposition are shown as solid lines. Dotted lines in the left and right columns correspond to decomposition without (1) and with (2) relaxation, respectively. $(v, L)(p, T)$ and $(v, L)(v, L)$ boundary conditions have been used in (a) and (b), respectively, the iteration procedure ( 4 cycles) has been applied in (c) with the $(v, L)(p, T)$ boundary condition.

2. Figure 7.3.b shows the result obtained using the $(v, L)(v, L)$ boundary condition. The latter is motivated by the discontinuity of the luminosity for the previously discussed $(v, L)(p, T)$ boundary condition. Rather than the considerable discontinuity of $L$ we expect a more tolerable discontinuity of its derivative for 
the $(v, L)(v, L)$ boundary condition. In fact, the results given in figure 7.3.b1 are satisfactory. After relaxation the relative error in the luminosity is of the order of $10^{-3}$ (figure 7.3.b2). However, in this case the error of $L$ directly enters the boundary conditions for the subsequent timestep and leads to an accumulation of the error at the interface. This problem can be removed partially by switching between different interfaces for subsequent timesteps. The remaining error can then spread sufficiently and does not influence the further integration significantly.

3. Figure 7.3.c shows the result obtained using the iteration procedure described in section 7.3.3. After four iteration cycles it is comparable to that obtained with the $(v, L)(v, L)$ boundary condition. By performing more iteration cycles the accuracy could be improved even more. However, the iteration procedure is computationally much more expensive than the alternatives discussed and therefore of no practical use.

We thus conclude, that using the domain decomposition procedure together with overlapping domains and $(v, L)(v, L)$ boundary conditions, and switching between different interfaces, yields satisfactory results at low computational cost.

\subsubsection{Validation of the domain decomposition procedure}

The domain decomposition procedure with overlapping domains and $(v, L)(v, L)$ boundary conditions has been compared for validation with the original approach using no decomposition. The comparison starts at $6.47 \cdot 10^{7} \mathrm{sec}$, i.e., well after the formation of the shock and the associated instability, and extends to $6.58 \cdot 10^{7} \mathrm{sec}$. The typical periods of unstable strange modes driving the pulsations of the star are of the order of $5 \cdot 10^{6}$ sec, whereas the modes carrying the stratification instabilities of the shock front have periods of $\sim 10^{5} \mathrm{sec}$. Therefore, the test covers approximately 0.2 periods of the overall envelope pulsations and 10 periods of shock oscillations.

Convergence and error control is done using the following criterion based on a $l^{2}$ norm:

$$
\frac{1}{N}\left(\sum_{i} f_{i}^{2}\right)^{\frac{1}{2}}<E
$$

where $f_{i}$ denotes the relative error of a physical quantity in gridpoint or cell $i$ and $N$ the total number of gridpoints. $E$ is the prescribed error bound and the sum extends

over all gridpoints. $f_{i}^{2}$ contains the weight-function of the $l^{2}$-norm which is chosen to be proportional to the mass of the corresponding cell, $f_{i}^{2} \propto m_{i}$. Thus the various regions of the star contribute to the error in a different way and domain decomposition using the same error bound in all domains will result in different accuracies for the various domains and compared with the approach without domain decomposition. Accordingly 

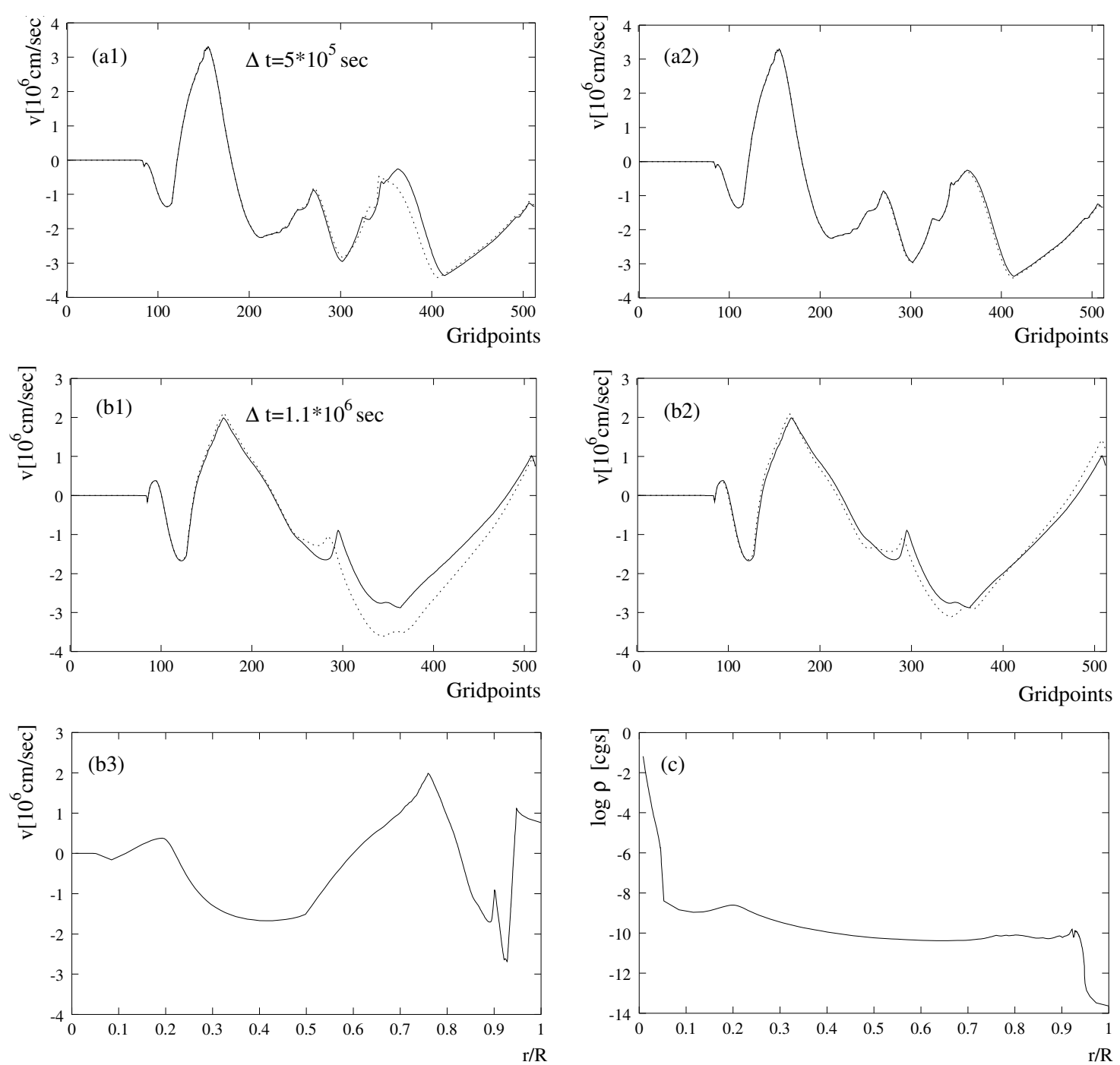

Figure 7.4: The velocity as a function of gridpoint with (dashed line) and without (solid line) domain decomposition after $5 \cdot 10^{5} \mathrm{sec}(\mathrm{a} 1)$ and $1.1 \cdot 10^{6} \mathrm{sec}(\mathrm{b} 1)$ of simulated time. All simulations start at $t=6.47 \cdot 10^{7} \mathrm{sec}$ (after the formation of the shock front) with the same initial model. In Figures (a2) and (b2) velocities obtained with decomposition are taken $10^{4} \mathrm{sec}(\mathrm{a} 2)$ and $5 \cdot 10^{4} \mathrm{sec}(\mathrm{b} 2)$ later than their counterparts without decomposition, respectively. This physically irrelevant phaseshift reduces the differences between the two approaches significantly. For comparison, the velocity and the density after $1.1 \cdot 10^{6}$ sec of simulated time are presented as a function of relative radius in figures (b3) and (c), respectively (without decomposition).

the error bound has to be adapted to the domains contribution to the numerical error. On the other hand, as an identical error control cannot be guaranteed, results with and without decomposition are expected to differ slightly.

In the following figures 7.4 and 7.5 , solid lines correspond to the approach without 

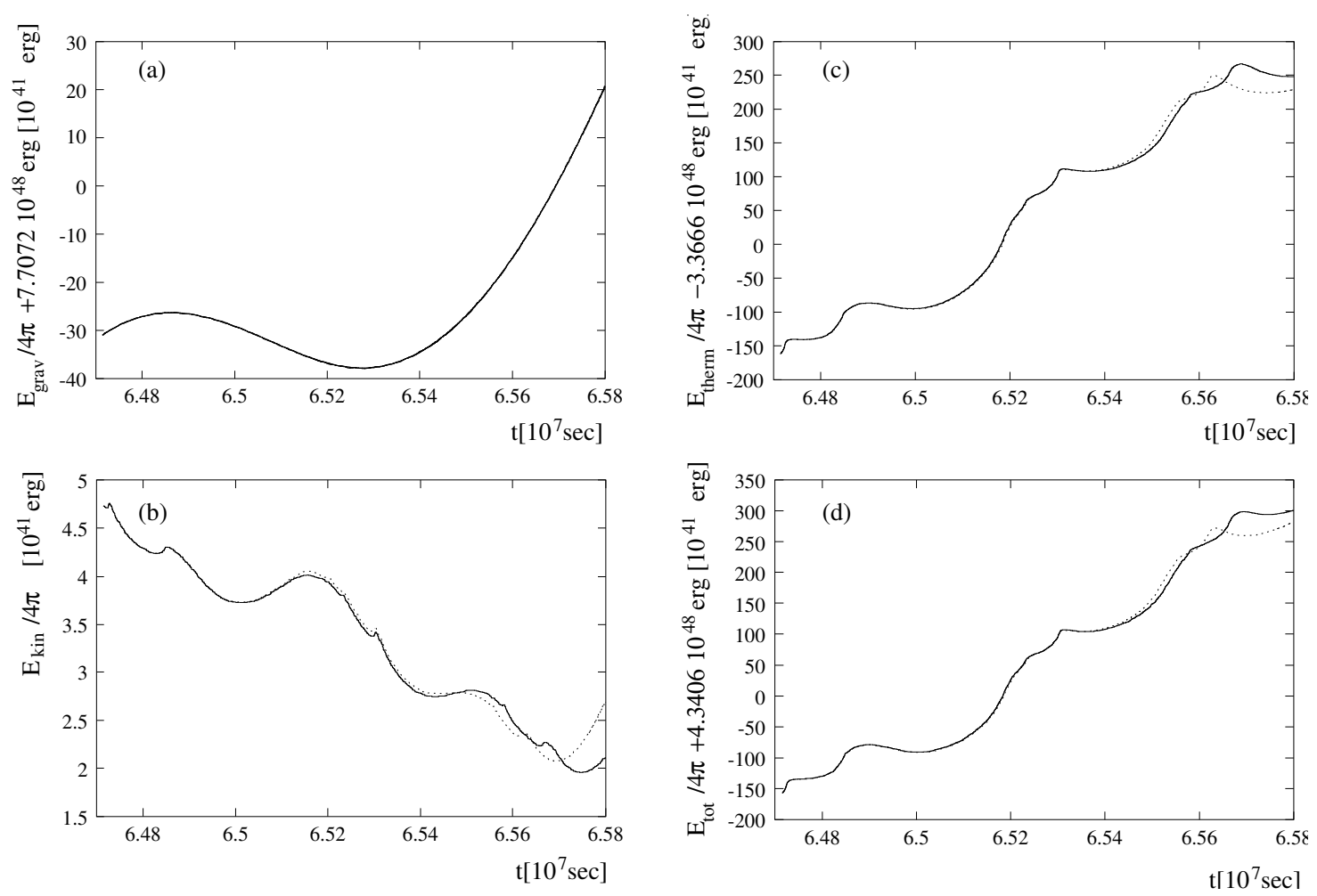

Figure 7.5: Gravitational (a), kinetic (b), thermal (c) and total (d) energy as a function of time. Solid and dashed lines correspond to simulations without and with domain decomposition, respectively.

decomposition, dotted lines refer to decomposition. Figure 7.4 shows the velocity $v$ as a function of gridpoint after $5 \cdot 10^{5} \mathrm{sec}$ (figure 7.4.a1) and $1.1 \cdot 10^{6} \mathrm{sec}$ (figure 7.4.b1) of simulated time, respectively. For comparison, the velocity and the density after $1.1 \cdot 10^{6}$ sec of simulated time are also presented as a function of relative radius in figures (b3) and (c), respectively (without decomposition). The shock is located at $r / R \approx 0.95$ and resolved by approximately 150 gridpoints. The high resolution of the shock zone is necessary to represent its oscillations (Grott, Glatzel \& Chernigovski 2003).

Considering $5 \cdot 10^{5} \mathrm{sec}$ of simulated time, domain decomposition yields excellent agreement with the original approach up to the position of the shock front (at grid point 320). Around the shock front the results differ slightly, whereas above it the agreement between the two approaches is again satisfying. The agreement may be found to be even better, if a phaseshift in time of about $10^{4} \mathrm{sec}$ between the results discussed is taken into account, i.e., if results of the original approach are compared with those obtained $10^{4}$ sec later with decomposition (figure 7.4.a2). We emphasise, that the time interval corresponds to five oscillation cycles of the shock front instability, and implies a phaseshift of only $\frac{\pi}{5}$. With respect to the fact that the phaseshift is physically irrelevant, we thus regard the agreement as fully satisfying.

After $1.1 \cdot 10^{6} \mathrm{sec}$ of simulated time the differences become more pronounced, in 
particular in the vicinity of the shock (now at gridpoint 300). Similar to the previous discussion, however, including a suitable phaseshift of $5 \cdot 10^{4} \mathrm{sec}$ a reasonable agreement may be achieved (Figure 7.4.b2).

Figure 7.5 shows the gravitational (Figure 7.5.a), kinetic (Figure 7.5.b), thermal (Figure 7.5.c) and total energy (Figure 7.5.d) of the envelope as a function of time. We note that the kinetic energy given in figure 7.5.b is seven orders of magnitude smaller than either the gravitational or thermal energy. It may be referred to as the energy of the pulsation and is therefore of central interest in the present context. Its variation with time is one order of magnitude smaller than that of the gravitational energy, which itself is one order of magnitude smaller than that of the thermal energy. Therefore the variation of the total energy is dominated by the thermal energy.

Domain decomposition reproduces the gravitational energy perfectly. This can be attributed to the use of overlapping domains, since without them considerable disagreement is found. The latter consists of oscillations of the solution around the curve given in Figure 7.5.a. With respect to kinetic (Figure 7.5.b), thermal (7.5.c) and total energy (7.5.d), the agreement is satisfactory up to the time $6.55 \cdot 10^{7} \mathrm{sec}$. The deviations at later times can be partially attributed to the phase shift discussed above.

To summarise, our comparisons prove the domain decomposition procedure to provide reliable and satisfactory results. We emphasise that domain decomposition violates the conservativity otherwise inherent in the numerical scheme. How this violation of conservativity contributes to the discrepancies discussed is an open question.

\subsubsection{Speed-Up of the calculations}

In order to estimate the speed-up achieved by the domain decomposition procedure, we assume that the number of iterations $I$ needed to solve the implicit equations with prescribed accuracy is not changed by decomposition, i.e., for convergence $I$ iterations are required in the shock region $\mathcal{D}_{s h}$ integrated with timestep $\tau$ and the same number of iterations $I$ are needed to integrate the domains $\mathcal{D}_{l}$ and $\mathcal{D}_{r}$ with time step $k \cdot \tau$. Moreover, we assume that $I$ iterations are required to integrate with timestep $\tau$ and without decomposition.

We denote the number of gridpoints by $N$ and the size of the domains $\mathcal{D}_{l}, \mathcal{D}_{s h}$ and $\mathcal{D}_{r}$ by $N_{l}, N_{s h}$ and $N_{r}$, respectively. ( $N_{l}, N_{s h}$ and $N_{r}$ include the overlap.) Integrating $k$ timesteps $\tau$ without decomposition then requires

$$
O_{1}=k \cdot N \cdot I
$$

operations. Decomposing the grid into two or three domains, we need for the integration of the same time interval

$$
\begin{aligned}
& O_{2}=N_{l} \cdot I+k \cdot N_{s h} \cdot I \quad \text { and } \\
& O_{3}=\left(N_{l}+N_{r}\right) \cdot I+k \cdot N_{s h} \cdot I
\end{aligned}
$$




\begin{tabular}{ccccc} 
Domains & Size of $\mathcal{D}_{s h}$ & Overlap & $\begin{array}{c}\text { Estimated } \\
\text { Speed-Up }\end{array}$ & $\begin{array}{c}\text { Measured } \\
\text { Speed-Up }\end{array}$ \\
\hline 3 & 160 & 16 & 2.75 & 2.42 \\
3 & 160 & 32 & 2.72 & 2.36 \\
3 & 192 & 16 & 2.34 & 2.15 \\
3 & 192 & 32 & 2.32 & 2.14 \\
2 & 272 & 16 & 1.77 & 1.61 \\
2 & 272 & 32 & 1.76 & 1.56 \\
2 & 288 & 16 & 1.68 & 1.67 \\
2 & 288 & 32 & 1.67 & 1.61 \\
\hline
\end{tabular}

Table 7.1: Estimated and measured speed-up for a grid with $N=512$ points and decomposition into 2 and 3 domains with two different overlaps.

operations, respectively. Thus we expect a speed-up of the calculations by decomposition by a factor of

$$
\begin{aligned}
& s_{2}=\frac{O_{1}}{O_{2}}=\frac{k N}{N_{l}+k N_{s h}} \quad \text { and } \\
& s_{3}=\frac{O_{1}}{O_{3}}=\frac{k N}{\left(N_{l}+N_{r}\right)+k N_{s h}},
\end{aligned}
$$

respectively. It essentially depends on $N_{s h}$ and $k$. For large $k$ it is approximately given by $N / N_{s h}$. However, for large $k, N_{s h}$ increases too (see section 7.3.2). A comparison between the estimated and measured speed-up is presented in Table 7.1. For the hypothetical case of large $k, N_{s h}=16$ and an overlap of 8 gridpoints we obtain a speed-up by a factor of 16 . This situation could in principle be realised for shock oscillations with smooth spatial structure which can be represented by a small number of gridpoints. This example demonstrates the power of the method when applied to a suitable situation.

Compared to the one dimensional case, a considerably higher speed-up is expected if decomposition is applied to two dimensional problems, since it reduces the size of matrices to be inverted, the latter being a $N^{2}$ operation in the worst case for iterative methods and the matrices considered.

\subsection{Application to a P Cygni model}

We apply the method discussed above to a stellar envelope model with parameters close to those observed for the star P Cygni. Concerning luminosity, effective temperature and chemical composition for this object, various authors (Najarro, Hillier \& Stahl 1997 and Pauldrach \& Puls 1990) agree that these parameters should lie in the vicinity of $L=752.5 \cdot 10^{3} L_{\odot}, T_{\text {eff }}=19300 K$ and $X=0.31, Y=0.67, Z=0.02$. The most uncertain parameter of P Cygni is its mass. Standard stellar evolution calculations indicate 

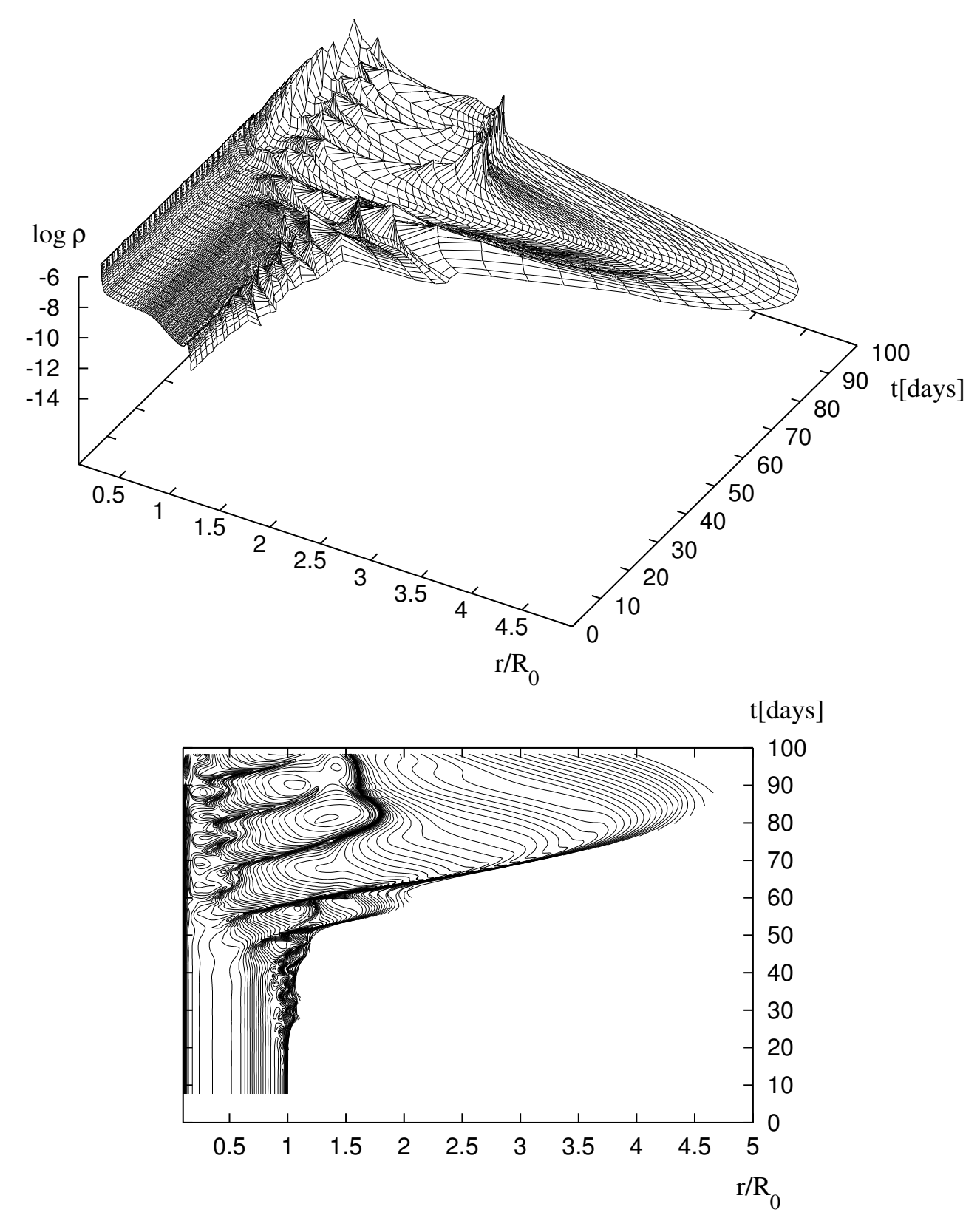

Figure 7.6: Density $\rho$ as a function of radius (in units of the initial radius) and time for an envelope model of P Cygni (top panel). The corresponding contour plot is given in the bottom panel. Strange mode instabilities, but no shock oscillations are resolved in these diagrams. 


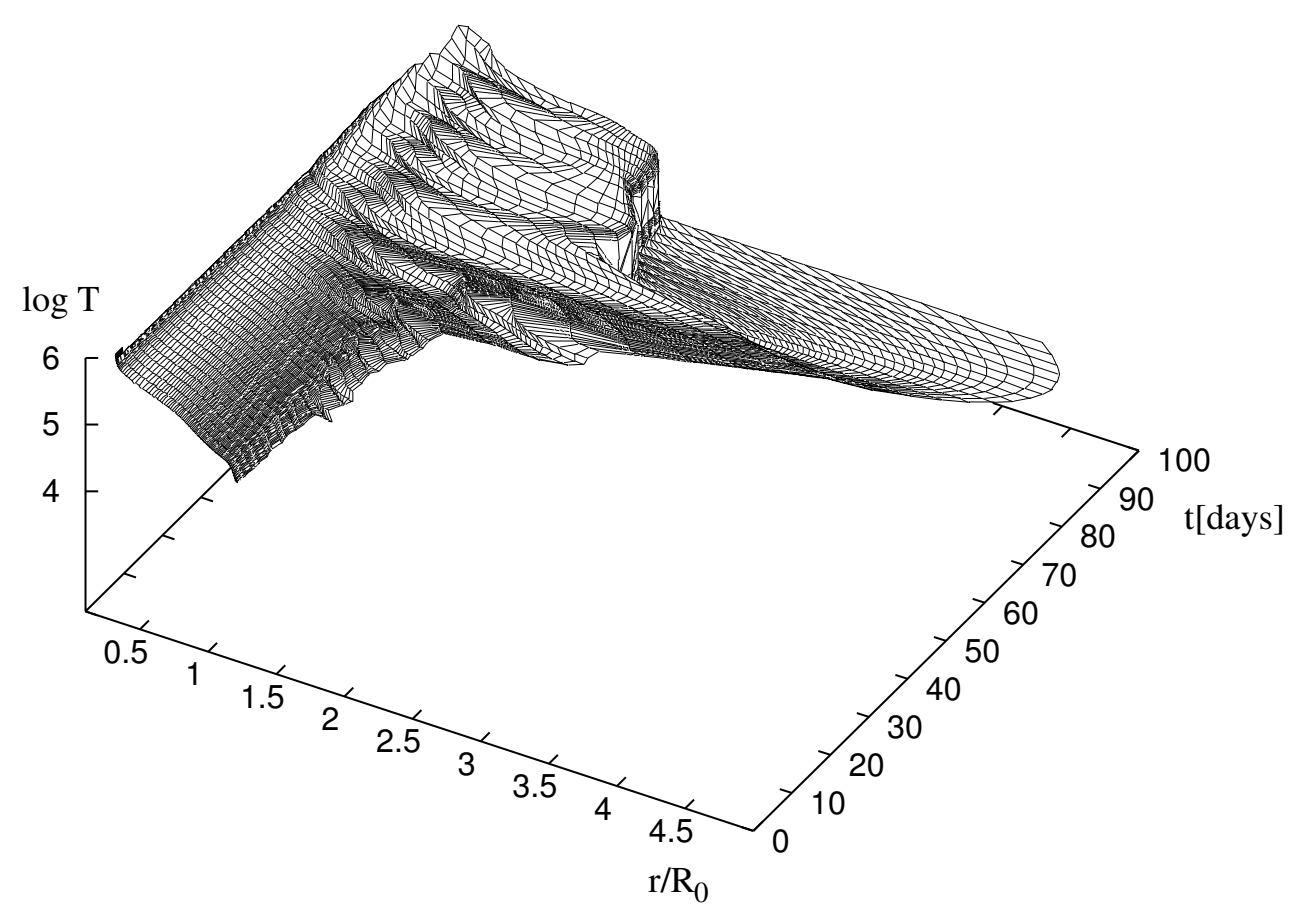

$\mathrm{t}$ [days]

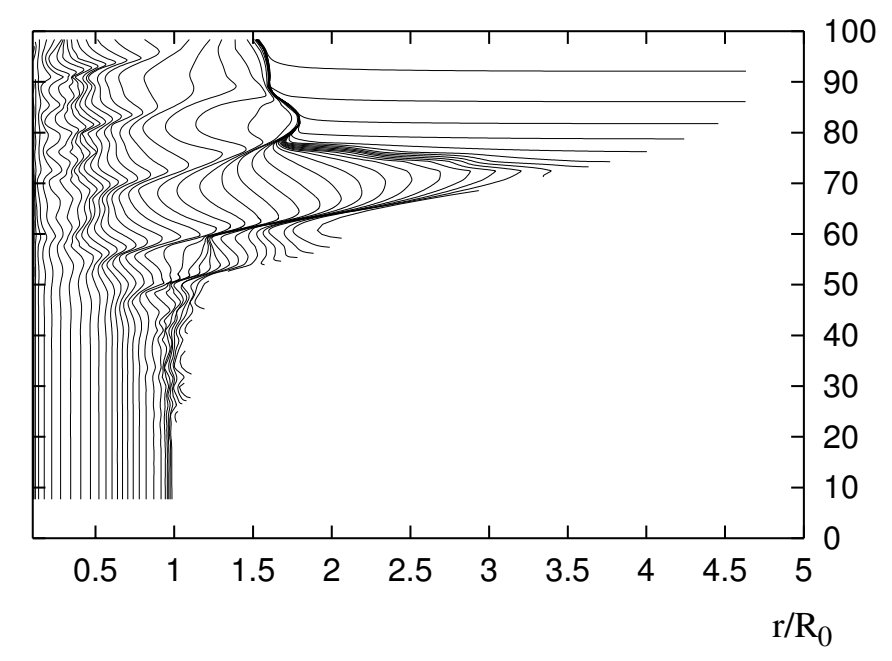

Figure 7.7: Same as Figure 7.6, but for the temperature $T$. 


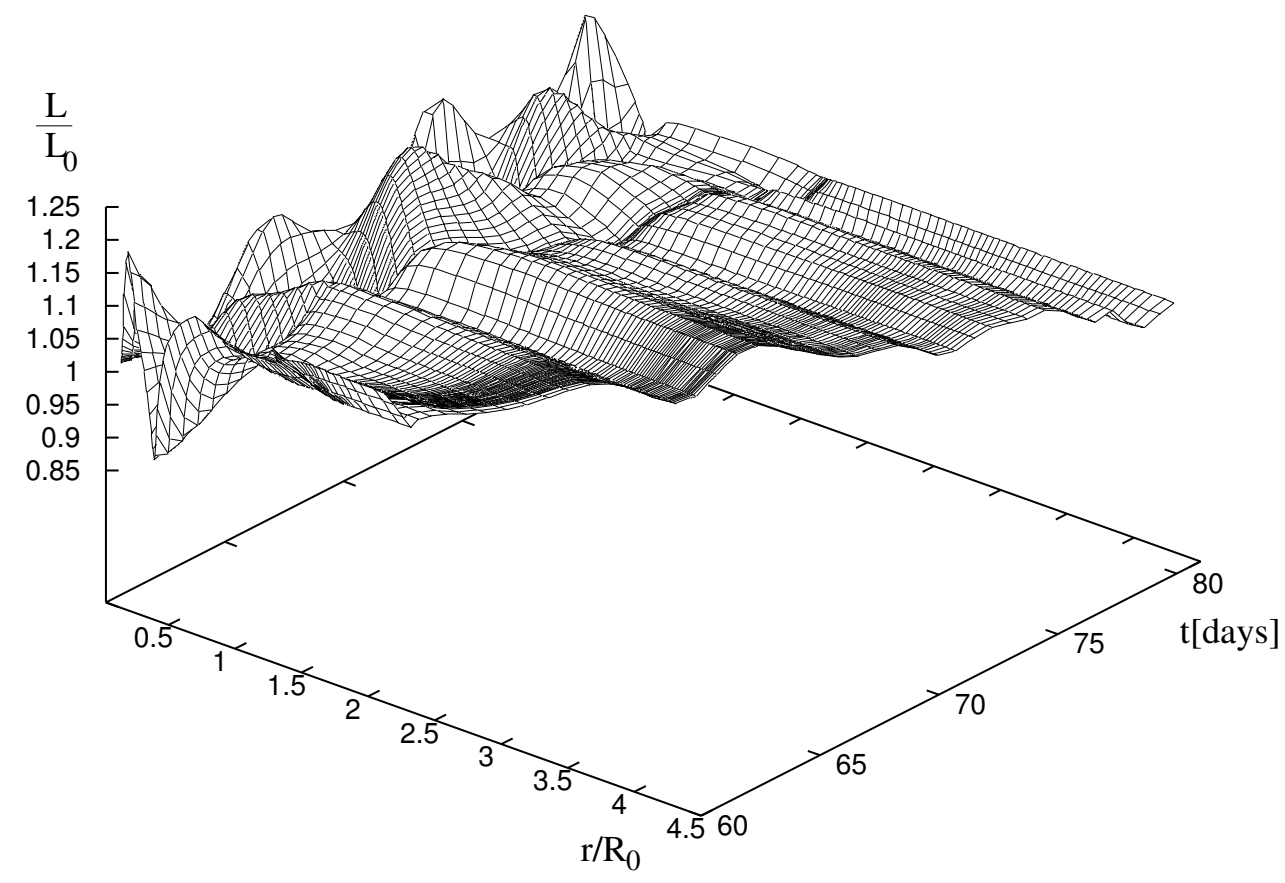

$\mathrm{t}$ [days]

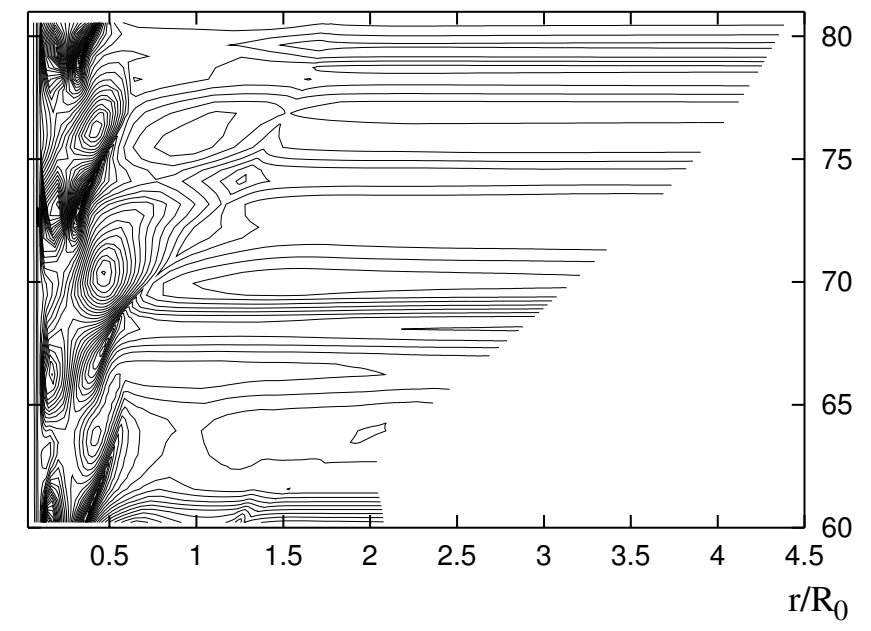

Figure 7.8: Luminosity in units of the initial luminosity as a function of radius (in units of the initial radius) before the shock capturing. The corresponding contour plot is given in the bottom panel. Note that the luminosity variations take place on the dynamical timescale. 

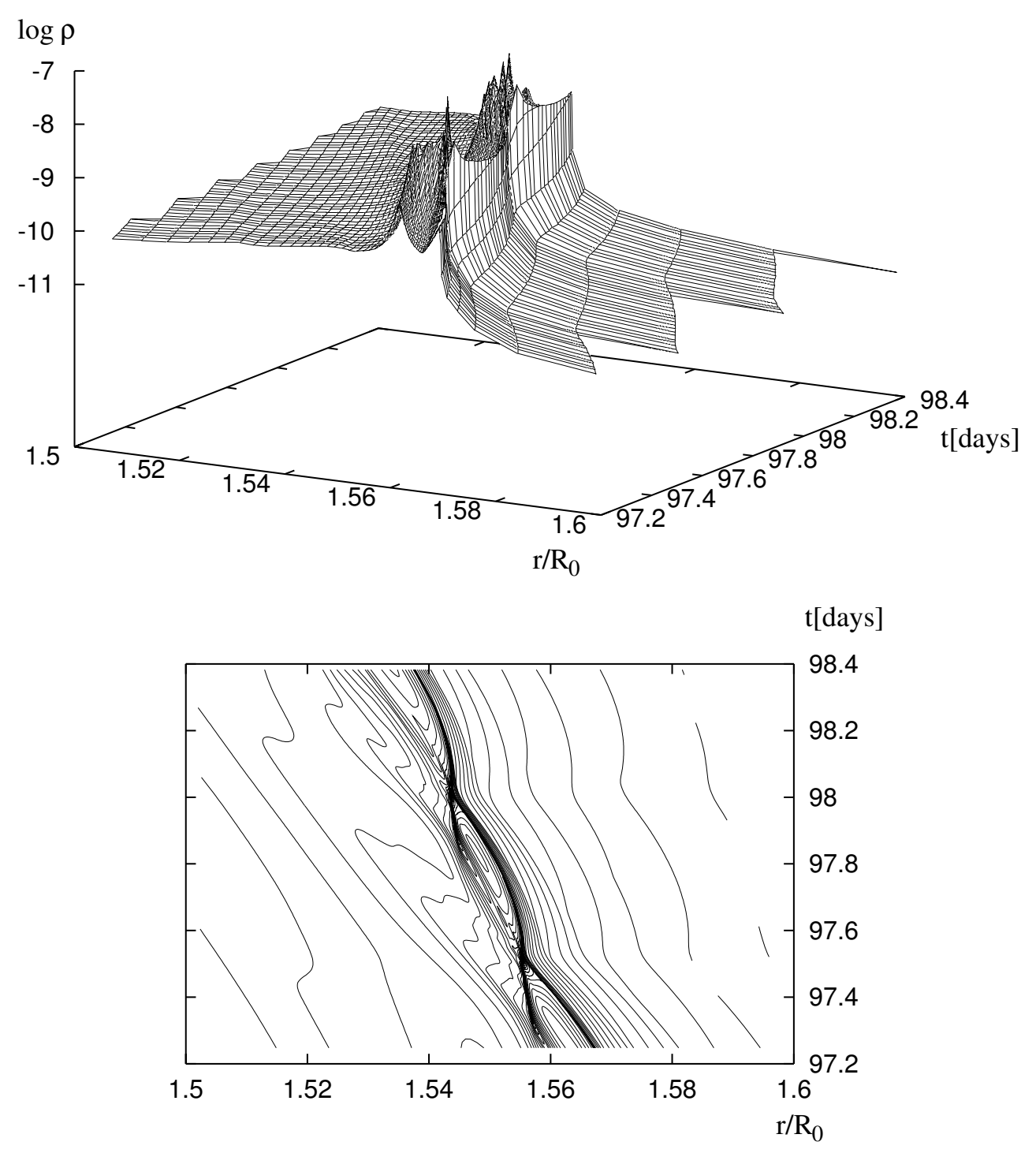

Figure 7.9: Same as Figure 7.6 in the vicinity of the captured shock and for a time interval appropriate to resolve the shock oscillations. 

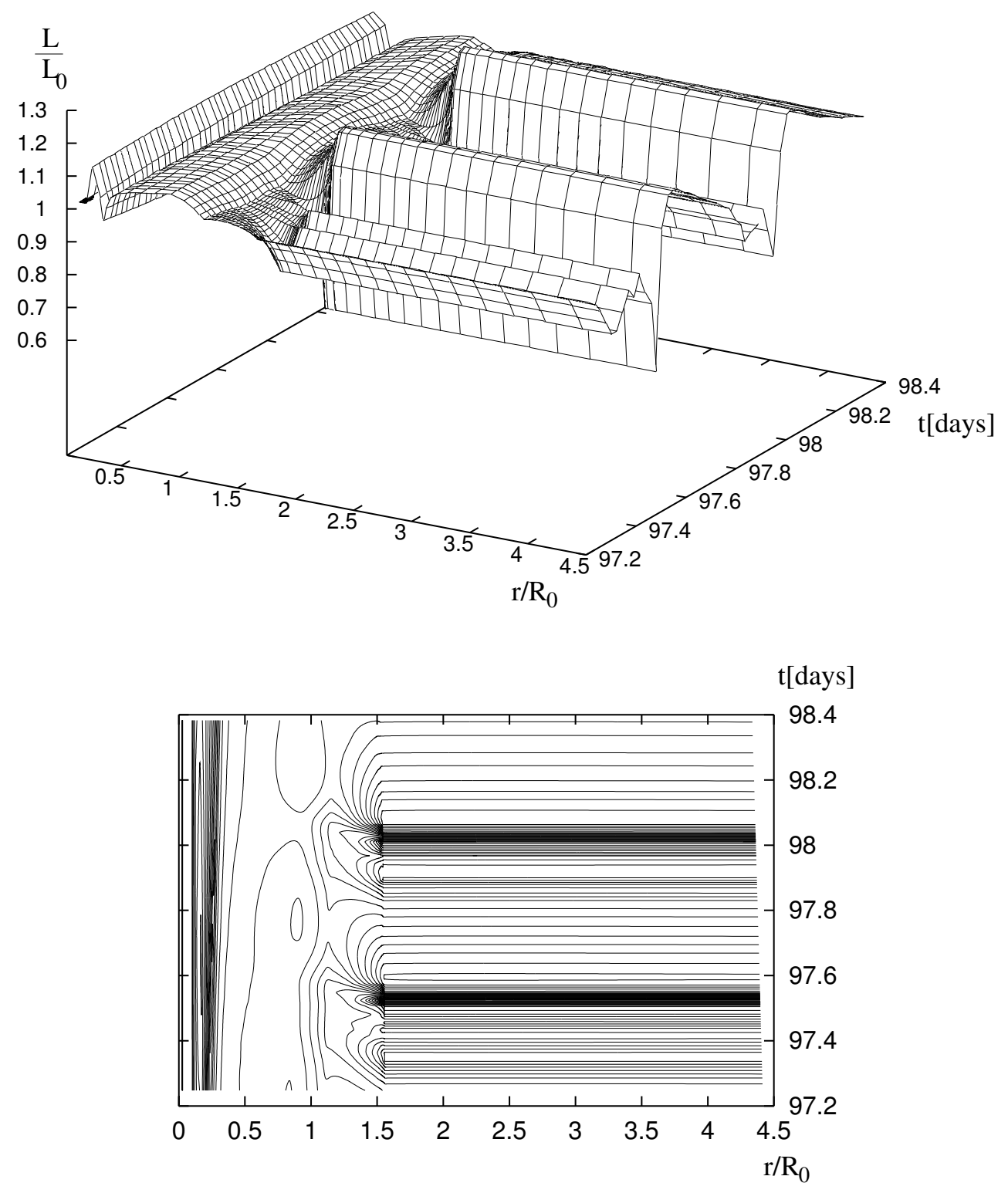

Figure 7.10: Luminosity in units of the initial luminosity as a function of radius (in units of the initial radius) and a time interval appropriate to resolve the shock oscillations (top panel). The corresponding contour plot is given in the bottom panel. Note that the rapid photospheric luminosity perturbations are defined at the captured shock (at $r / R_{0} \sim 1.5$ ). Below the shock, luminosity perturbations are governed by strange mode instabilities operating on the dynamical timescale. 
a mass of $M=50 M_{\odot}($ El Eid \& Hartmann 1993), whereas spectroscopic observations are consistent with masses down to $M=23 M_{\odot}$ (Pauldrach \& Puls 1990). For our simulations we adopt $M=26.5 M_{\odot}$, a value supported by the observation. Even with a more conservative mass of $M=50 M_{\odot}$, the model is known to be unstable with respect to strange modes (Kiriakidis, Fricke \& Glatzel 1993). The higher value of $L / M$ adopted here amplifies the tendency towards instability through shorter thermal timescales.

The simulation of strange mode instabilities in P Cygni starts from the envelope model in hydrostatic equilibrium without any external perturbations. Strange mode instabilities develop from numerical noise, pass the linear regime of exponential growth and form multiple shocks in the non-linear domain. One of these shocks is captured in the $\mathrm{H}$-ionisation zone and starts oscillating on timescales of the order of the sound travel time across the front $(\sim 0.5$ days $)$, whereas its mean position varies on the dynamical timescale ( $\sim 10$ days). In this phase of the evolution on two different timescales domain decomposition is used to speed up the calculation considerably.

Figure 7.6 shows the density $\rho$ as a function of radius (in units of the initial radius) and time for the envelope model of P Cygni. The corresponding contour plot is given in the bottom panel. Note, that contour lines here and in the following are not always closed, since during the evolution of the star e.g. the density drops to values lower than those of the initial model. After reaching the non-linear regime at $t=\sim 20$ days, shocks are formed in the outer envelope, travelling outwards and inflating the envelope successively up to 4.5 initial radii. During this period, the surface velocity reaches 55 per cent of the escape velocity at $t=75$ days. After $\sim 80$ days the envelope starts to collapse, and a shock originating at $r / R \approx 0.6$ at time $t=70$ days is then captured in the H-ionisation zone around $r / R \approx 1.5$ at $t \approx 85$ days. Subsequent shocks, generated by the primary strange mode instability, are confined to the region below the captured shock.

Figure 7.7 is the analogue to Figure 7.6 for the temperature $T$. The shocks discussed above can easily be identified in the contour plot. Note the steep temperature gradient at the captured shock after its formation.

Figure 7.8 shows the luminosity in units of the initial luminosity as a function of radius (in units of the initial radius) and a time interval before the shock capturing. The luminosity varies on the dynamical timescale by 10 per cent, corresponding to a bolometric variation of $\sim 0.1^{\mathrm{m}}$. It is defined in the inner envelope and remains constant with respect to radius above. There, luminosity perturbations cannot be sustained due to the low specific heat and the associated short thermal timescales. These luminosity variations remind of the microvariations in P Cygni, described by, e.g., de Groot, Sterken \& van Genderen (2001). These authors report on cyclic behaviour of the visual brightness with amplitudes of $\sim 0.1^{\mathrm{m}}$ and cycle lengths between 10 and 25 days, best fitting a quasi-period of $17.3^{\mathrm{d}}$.

Figure 7.9 shows the density $\rho$ as a function of radius (in units of the initial radius) in the vicinity of the captured shock and a time appropriate to resolve the shock oscillations. In particular, the corresponding contour plot in the bottom panel shows variation on two different timescales. It exhibits both shock oscillations with a mean period of $\sim 0.5$ days and the evolution on the dynamical timescale, as indicated by the variation of the shock 
position between $r / R_{0} \approx 1.56$ and $r / R_{0} \approx 1.54$.

Figure 7.10 shows the luminosity in units of the initial luminosity as a function of radius (in units of the initial radius) and a time interval appropriate to resolve the shock oscillations. The rapid photospheric luminosity perturbations are defined at the captured shock (at $r / R_{0} \sim 1.5$ ) and remain constant above it (see discussion of Figure 7.8). The variation amounts to 20 per cent, corresponding to $\sim 0.2^{\mathrm{m}}$ bolometrically. Below the shock, luminosity perturbations are governed by strange mode instabilities operating on the dynamical timescale. The photometric luminosity perturbation is therefore a superposition of two effects, dominated by the fast oscillations induced by the shock. We emphasise, however, that the one dimensional calculations presented here have to be interpreted with caution, since massive stars are known to suffer also from non-radial instabilities (Glatzel \& Mehren 1996). Whether the captured shocks survive the deformations induced by non-radial instabilities or become unstable themselves with respect to non-radial perturbations, remains to be tested by at least two dimensional calculations. They might break apart and could then contribute to the entire variation by stochastically adding high-frequency perturbations to the cyclic perturbations on the dynamical timescale induced by strange mode instabilities. On the other hand, from the observations discussed by de Groot et al. (2001), no indications for the stability properties of the captured shock found here can be inferred, since the time resolution of the data is not sufficiently high ( $\sim$ one measurement per day).

Another effect of the primary strange mode instability consists of a mass transfer from the instability region into the outer parts of the stellar envelope. Owing to the Lagrangian approach chosen here, this implies a reduced spatial resolution in the inner part of the stellar envelope. Simultaneously, grid points are concentrated around the captured shock. For reliable calculations, however, a high resolution of the instability region is indispensable. Otherwise, the physical strange mode instability generating acoustic energy and shock waves is suppressed. Thus, due to insufficient resolution, the simulation discussed had to be stopped after $\sim 100$ days of simulated time. To overcome the difficulty grid reconstruction is necessary. A corresponding algorithm, consistently inserting and eliminating gridpoints during the calculation is currently being developed and will be commented on in a forthcoming publication.

\subsection{Conclusions}

Motivated by the discovery of high frequency shock oscillations in pulsating stars confined in a narrow region, we have developed a procedure for an efficient treatment of such phenomena. The integration domain is decomposed into several subdomains according to the various, vastly different timescales present in the configuration. To save computing time, these domains are integrated according to their respective timescales.

Criteria for the choice and decomposition of the computational domains have been derived. Decomposition implies artificial inner boundaries which require suitable boundary conditions. How these have to be chosen in order to minimise the numerical error 
(compared to the approach without decomposition) has been discussed. An overlap of the domains was found to be necessary to produce satisfactory results.

The decomposition technique has been tested and validated by comparison with results obtained without decomposition. The major effect of decomposition was found to consist of a delay in time (phaseshift) with respect to the original calculation. The latter is regarded as physically largely irrelevant. Otherwise the numerical quality of the results was proven to be satisfactory.

For the models considered, decomposition was found both theoretically and by numerical tests to reduce the computational costs by approximately a factor of two. The speed-up critically depends on the size of the rapidly varying domain. Although a reduction of computing time by 50 per cent sounds moderate, it is of practical relevance considering the duration of several weeks for a complete simulation. The intended extension of the procedure to two dimensional problems will yield an appreciably higher speed-up than that for the one dimensional model considered here, since the iterative inversion of some matrices there requires of the order of $N^{2}$ operations. Moreover, decomposition in two dimensions may reduce the size of matrices to be inverted such that fast direct methods (requiring of the order of $N$ operations) become most efficient, whose application would then imply a further acceleration of the computation.

We have applied the method to a model for the star P Cygni, paying special attention to the adequate treatment of the different timescales involved.

\section{Acknowledgements}

We thank Professors K.J. Fricke and G. Warnecke for encouragement and support. Financial support by the Graduiertenkolleg "Strömungsinstabilitäten und Turbulenz" (MG) and by the DFG under grant WA 633 12-1 (SC) is gratefully acknowledged. The numerical computations have been carried out using the facilities of the GWDG at Göttingen and of the ZIB at Berlin. 


\section{Bibliography}

Ardelyan N., Gushchin I., 1982, Moscow Univ. Comp. Math. and Cybernetics, 3, 3

Baker N.H., Kippenhahn R., 1965, ApJ, 142, 869

Cox J.P., 1980, Theory of Stellar Pulsation. Princeton Univ. Press, Princeton, NJ

Dorfi E. A., Gautschy A., 2000, ApJ, 545, 982

El Eid M.F., Hartmann D., 1993, ApJ, 404, 271

Fraley, G.S., 1968, Ap\&SS, 2, 96

Glatzel W., Kiriakidis M. 1993, MNRAS, 263, 375

Glatzel W., Kiriakidis M., Chernigovskij S., Fricke K.J. 1999, MNRAS, 303, 116

Glatzel W., Mehren S. 1996, MNRAS, 282, 1470

de Groot M., Sterken C., van Genderen A.M., 2001, A\&A, 376,224

Grott M., Glatzel W., Chernigovski S., 2003, MNRAS, submitted

Humphreys R.M., Davidson K., 1979, ApJ, 232, 409

Humphreys R.M., Davidson K., 1994, PASP, 106, 704

Iglesias C.A., Rogers F.J., Wilson B.G., 1992, ApJ, 397, 717

Kiriakidis M., Fricke K.J., Glatzel W. 1993, MNRAS, 264, 50

Najarro F., Hillier D.J., Stahl O., 1997, A\&A, 326, 1117

Pauldrach A.W.A., Puls J., 1990, A\&A, 237, 409

Rogers F.J., Iglesias C.A., 1992, ApJS, 79, 507

Samarskii A., Popov Yu., 1969, Zh. Vychisl. Mat. i Mat. Fiz., 9, 953

Samarskii A., Tishkin V., Favorskii A., Shashkov M., 1981, Diff. Eqns., 17, 854

Shashkov M., 1996, Conservative Finite-Difference Methods on General Grids, CRC

Press, New York

Wu Z., 1999, SIAM J. Sci. Comput., 20, 5, 1851

Wu Z., Zou H., 2000, J. Comp. Phys., 157, 2 


\title{
8. Grid reconstruction
}

\section{A grid reconstruction procedure for the simulation of stel- lar instabilities ${ }^{1}$}

\author{
S. Chernigovski ${ }^{3}$, M. Grott ${ }^{2}$ and W. Glatzel ${ }^{2}$ \\ ${ }^{2}$ Universiẗ̈ts-Sternwarte Göttingen, Geismarlandstr .11, 37083 Göttingen, Germany \\ ${ }^{3}$ Institut für Analysis und Numerik, Universität Magdeburg, Universitätsplatz 2, 39106 Magdeburg, Germany
}

\begin{abstract}
The simulation of stellar instabilities requires a sufficient resolution of shocks, ionisation zones and instability regions. Resolution problems in the latter occur in simulations of the evolution of strange mode instabilities, if a Lagrangian description is used and the instability leads to a mass transfer in the stellar envelope. This necessitates a reconstruction of the Lagrangian grid. A grid reconstruction procedure suitable for the simulation of stellar instabilities and pulsations is presented and verified. This includes a systematic study of the influence of grid resolution and artificial viscosity on the result of numerical calculations.
\end{abstract}

Key words: hydrodynamics - instabilities - stars: mass-loss stars: oscillations - stars: variables: other.

\subsection{Introduction}

Sufficiently luminous objects, such as massive stars, are known to suffer from strange mode instabilities with growth rates in the dynamical range (Kiriakidis, Fricke \& Glatzel 1993; Glatzel \& Kiriakidis 1993). The boundary of the domain in the HertzsprungRussel diagram (HRD) above which all stellar models are unstable - irrespective of their metallicity -, coincides with the observed Humphreys-Davidson (HD) limit (Humphreys \& Davidson 1979). Moreover, the range of unstable models covers the stellar parameters

\footnotetext{
${ }^{1}$ This paper has been submitted to MNRAS (Chernigovski et al. 2003)
} 
for which the LBV phenomenon is observed (see, e.g., Humphreys \& Davidson 1994 and van Genderen 2001).

The high growth rates of the instabilities indicate a connection to the observed mass loss of the corresponding objects. To verify this suggestion, simulations of their evolution into the non linear regime have been performed. In fact, for selected models Glatzel et al. (1999) found the maximum velocity to exceed the escape velocity (see, however, Dorfi \& Gautschy 2000).

For a further study of a possible connection between strange mode instabilities and mass loss, we have constructed a stellar envelope model with mass $M=23 M_{\odot}$, effective temperature $T_{\text {eff }}=19300 \mathrm{~K}$, luminosity $L=752.5 \cdot 10^{3} L_{\odot}$ and chemical composition $X=0.31, Y=0.67, Z=0.02$. These values correspond to the parameters of the LBV P Cygni as derived by Pauldrach \& Puls (1990) and Najarro et al. (1997). The model suffers from violent strange mode instabilities which generate sound waves and shocks in the interior of the stellar envelope and transfer mass into the outer regions. We take this as an indication for an outburst of the object associated with mass loss. However, due to the Lagrangian description adopted the grid resolution in the instability region around $\log T \approx 5.3$ is decreased by the mass transfer. As a consequence of insufficient resolution the instability is suppressed and the mass flow ceases. With respect to the anticipated connection between strange mode instabilities and mass loss, a solution of this numerically induced problem is crucial. A grid reconstruction procedure which guarantees a sufficient resolution in the instability region is therefore necessary.

Subject of this paper is the development and validation of a grid reconstruction procedure satisfying the requirements discussed above. The basic equations and assumptions are introduced in Section 7.2 and a detailed motivation for the study is presented in Section 8.2. The influence of grid resolution and artificial viscosity on the simulation of the evolution of strange mode instabilities into the non-linear regime is studied in Sections 8.3.2 and 8.3.3. The grid reconstruction procedure is discussed in detail in Section 8.3.4 and verified in Section 8.3.5. Our conclusions follow.

\subsection{Basic equations and assumptions}

\subsubsection{Initial models and relaxation}

Hydrostatic envelope models are constructed for given mass $M$, effective temperature $T_{\text {eff }}$, luminosity $L$ and chemical composition $X, Y, Z$ by integration of the initial value problem posed by the equations of hydrostatic equilibrium, mass conservation and energy transport. Convection is treated in the standard mixing-length approach with 1.5 pressure scaleheights for the mixing length. The onset of convection is determined by the Schwarzschild criterion. For the opacities, OPAL tables (Iglesias, Rogers \& Wilson 1992; Rogers \& Iglesias 1992) are used. In an envelope model the stellar core and energy generation there are disregarded. For the subsequent dynamical calculations, the detailed treatment of the stellar core is replaced by imposing suitable boundary condi- 
tions at the bottom of the envelope. This is justified, since the core remains unaffected by the instabilities investigated here.

The envelope integration provides a spatial distribution of gridpoints that is is in general not compatible with the numerical scheme used in the dynamical calculations. Moreover the number of gridpoints is too high. Therefore the hydrostatic envelope model and its dependent variables have to be adapted to a grid suitable for the dynamical calculations. Otherwise the numerical errors induce perturbations with amplitudes in the non-linear regime. The problem may be overcome by damping the artificial perturbations using dissipation and viscosity or by a pseudo time evolution of the grid (Dorfi \& Drury 1987; Dorfi \& Feuchtinger 1995; Dorfi 1998). Using these techniques, however, the stellar models have to be perturbed externally to trigger the physical instability and envelope pulsations. Typically, initial velocity amplitudes of the order of $10 \mathrm{~km}$ $\mathrm{sec}^{-1}$ amounting to 10 per cent of the local sound speed are required (see, e.g., Dorfi \& Gautschy 2000).

In our approach the adaption of the hydrostatic envelope model to a suitable grid is achieved by reintegrating the envelope using the same difference scheme as that applied for the dynamical calculations and using the results of the standard envelope integration as initial guesses for the integration procedure. It implies, that - apart from the initial values - only the convective flux is taken from the predetermined envelope model. Using this approach, hydrostatic equilibrium is satisfied to within round-off errors, rather than to $\mathcal{O}\left(\frac{1}{N}\right)$, which is the difference between incompatible difference schemes $(N$ is the number of gridpoints). Thus, no damping of artificial numerically induced perturbations is required. Physical instabilities and stellar pulsations then develop without any further (unphysical) external perturbation from numerical noise, which (for the models considered here) corresponds to velocities of the order of a few $\mathrm{cm} \mathrm{sec}^{-1}$.

\subsubsection{Non-linear evolution}

The evolution of instabilities of a stellar envelope is followed into the non-linear regime assuming spherical symmetry and adopting a Lagrangian description. The independent variables are the time $t$ and the Lagrangian mass coordinate $s$ which we define by $d s=$ $\rho(r, 0) r^{2} d r$. The equations to be solved (see, e.g., Cox 1980) are then given by mass conservation,

$$
\frac{\partial}{\partial t}\left(\frac{1}{\rho}\right)=\frac{\partial\left(r^{2} v\right)}{\partial s}
$$

momentum conservation,

$$
\frac{\partial v}{\partial t}=-r^{2} \frac{\partial p}{\partial s}-\frac{4 \pi G M_{r}}{r^{2}}
$$

energy conservation

$$
\frac{\partial \epsilon}{\partial t}=-p \frac{\partial\left(r^{2} v\right)}{\partial s}-\frac{\partial\left(r^{2}\left(F_{\mathrm{rad}}+F_{\mathrm{con}}\right)\right)}{\partial s}
$$


and the diffusion equation for energy transport

$$
F_{\text {rad }}=-\theta r^{2} \frac{\partial T}{\partial s}
$$

where $\rho, p, T, r, v$ and $\epsilon$ denote density, pressure, temperature, radius, velocity and specific internal energy, respectively. We emphasise that $\frac{\partial}{\partial t}$ is the substantial time derivative. $M_{r}$ is the mass inside a sphere of radius $r, G$ the gravitational constant and

$$
\theta=\frac{4 a c T^{3}}{3 \kappa \rho}
$$

is the diffusion coefficient, which contains the radiation constant $a$, the speed of light $c$ and the opacity $\kappa$. For consistency with the hydrostatic envelope integration, $\kappa$ is again taken from the OPAL tables (Iglesias, Rogers \& Wilson 1992; Rogers \& Iglesias 1992). $F_{\text {rad }}$ and $F_{\text {con }}$ are the radiative and the convective flux, respectively. The latter is kept constant during the evolution and equal to its initial value, in accordance with the standard frozen in approximation (see Baker \& Kippenhahn 1965). In this study we consider only models where this approximation is satisfied, i.e., where the turnover timescale of convection is much larger than the dynamical timescale and the fraction of energy transported by convection is not significant. The system of equations is closed by the definition of the velocity $v=\frac{\partial r}{\partial t}$ and the equations of state $p=\mathcal{P}(\rho, T)$ and $\epsilon=$ $\mathcal{E}(\rho, T)$. For consistency, the latter have to be identical to those used for the construction of the initial model.

Since the instabilities are localised in the outer envelope, the evolution of the core can be neglected. Its properties are taken into account by imposing time independent boundary conditions (i.e., by prescribing luminosity, [vanishing] velocity $v$ and [constant] radius) at the bottom of the envelope. At the outer boundary, the gradient of heat sources is required to vanish:

$$
\operatorname{grad}(\operatorname{div} F)=0
$$

This boundary condition implies (by using equations 8.1,8.3 and 8.4) boundary values for the temperature $T$ and the pressure $p$. It is chosen to ensure that outgoing shocks pass through the boundary without reflection.

The numerical code relies on a Lagrangian, with respect to time implicit, fully conservative difference scheme proposed by Fraley (1968) and Samarskii \& Popov (1969), which will be discussed in detail in the following section. To handle shock waves, artificial viscosity is used. Concerning tests of the code, we adopted the same criteria as Glatzel et al. (1999).

\subsubsection{Difference equations}

The equations governing the evolution of instabilities in the stellar envelope are discretised on a staggered mesh such that the radius $r$, the velocity $v$, the radiative and 
convective flux $F_{\text {rad }}$ and $F_{\text {con }}$ and the mass $M_{r}$ inside a sphere of radius $r$ (including the mass of the core) are defined on the nodes of the grid, whereas the density $\rho$, the pressure $p$, the specific internal energy $\epsilon$, the temperature $T$ and the diffusion coefficient $\theta$ are defined in the cells. In this scheme, equations 8.1- 8.4 may be written as

$$
\begin{aligned}
\left(\frac{1}{\hat{\rho}_{i}}-\frac{1}{\rho_{i}}\right) \cdot \frac{1}{\tau}= & \frac{\left(R_{i+1} \cdot v_{i+1}^{(0.5)}-R_{i} \cdot v_{i}^{(0.5)}\right)}{m_{i}} \\
\frac{\hat{v}_{i}-v_{i}}{\tau}= & -R_{i} \cdot \frac{\left(g_{i}^{(\alpha)}-g_{i-1}^{(\alpha)}\right)}{\bar{m}_{i}}-\frac{4 \pi G M_{r_{i}}}{\hat{r}_{i} r_{i}} \\
\frac{\hat{\epsilon}_{i}-\epsilon_{i}}{\tau}= & -g_{i}^{(\alpha)} \cdot \frac{\left(R_{i+1} \cdot v_{i+1}^{(0.5)}-R_{i} \cdot v_{i}^{(0.5)}\right)}{m_{i}} \\
& -\frac{R_{i+1} \cdot\left(F_{\mathrm{rad}, \mathrm{i}+1}^{(\sigma)}+F_{\mathrm{con}, \mathrm{i}+1}\right)}{m_{i}} \\
& +\frac{R_{i} \cdot\left(F_{\mathrm{rad}, \mathrm{i}}^{(\sigma)}+F_{\mathrm{con}, \mathrm{i}}\right)}{m_{i}} \\
F_{\mathrm{rad}, \mathrm{i}}= & -\bar{\theta}_{i} \cdot r_{i}^{2} \cdot \frac{T_{i}-T_{i-1}}{\bar{m}_{i}}
\end{aligned}
$$

$i$ denotes the number of the node/cell, with $i$ increasing outwards. $m_{i}=\rho_{i}\left(r_{i}^{3}-r_{i-1}^{3}\right) / 3$ is the mass of cell $i$ and $\bar{m}_{i}=\frac{1}{2} \cdot\left(m_{i}+m_{i-1}\right)$ the mass associated with node $i$, respectively. $g$ is the sum of pressure $p$ and artificial viscosity $Q$. The latter is given by

$$
Q= \begin{cases}C_{0} \rho(\operatorname{div} v)^{2} & \operatorname{div} v<0 \\ 0 & \operatorname{div} v \geq 0\end{cases}
$$

with the viscosity parameter $C_{0}>0$.

$\bar{\theta}_{i}=\sqrt{\theta_{i-1}\left(p_{i-1}, T_{i-1}\right) \cdot \theta_{i}\left(p_{i}, T_{i}\right)}$ defines the diffusion coefficient on the nodes of the grid. $R_{i}$ is given by

$$
R_{i}=\frac{1}{3}\left(\hat{r}_{i}^{2}+\hat{r}_{i} r_{i}+r_{i}^{2}\right)
$$

The definition of the velocity

$$
\frac{\hat{r}_{i}-r_{i}}{\tau}=v_{i}^{(0.5)}
$$



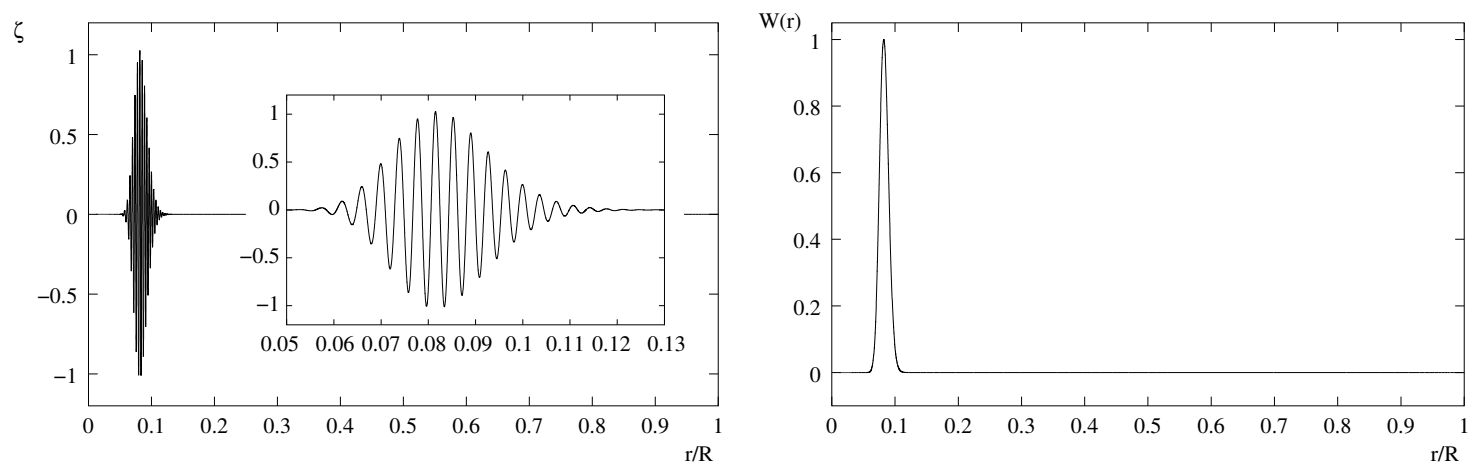

Figure 8.1: Lagrangian displacement $\zeta$ as a function of relative radius for the mode having $\omega_{R}=2.133 \cdot 10^{-4} \mathrm{sec}^{-1}, \omega_{I}=-3.149 \cdot 10^{-5} \sec ^{-1}$ (a). The corresponding work integral as a function of relative radius is given in (b).

and the equations of state

$$
\begin{aligned}
p_{i} & =\mathcal{P}\left(\rho_{i}, T_{i}\right) \\
\epsilon_{i} & =\mathcal{E}\left(\rho_{i}, T_{i}\right)
\end{aligned}
$$

close the system of equations.

The scheme is implicit with respect to time where quantities $\hat{x}$ and $x$ refer to the time $t_{n+1}$ and $t_{n}$, respectively. ( $t_{n+1}=t_{n}+\tau, \tau$ is the variable integration timestep.) $\alpha$ and $\sigma \in[0,1]$ are free weight parameters, and time averaging is done according to

$$
x^{(\alpha)}=\alpha \cdot \hat{x}+(1-\alpha) \cdot x
$$

In the presented simulations, the values $\alpha=\sigma=1$ have been adopted.

\subsection{Grid reconstruction}

\subsubsection{Motivation}

We have constructed a stellar envelope model with parameters appropriate for the star $\mathrm{P}$ Cygni. Concerning luminosity, effective temperature and chemical composition for this object, various authors (Najarro, Hillier \& Stahl 1997 and Pauldrach \& Puls 1990) agree that these parameters should lie in the vicinity of $L=752.5 \cdot 10^{3} L_{\odot}, T_{\text {eff }}=19300 \mathrm{~K}$ and $X=0.31, Y=0.67, Z=0.02$. The most uncertain parameter of P Cygni is its mass. Standard stellar evolution calculations indicate a mass of $M=50 M_{\odot}(\mathrm{El}$ Eid \& Hartmann 1993), whereas spectroscopic observations are consistent with masses down to $M=23 M_{\odot}$ (Pauldrach \& Puls 1990). For the simulations we have adopted $M=23 M_{\odot}$, the value supported by the observation. Even with a more conservative mass of $M=50 M_{\odot}$, the model is known to be unstable with respect to strange mode instabilities (Kiriakidis, Fricke \& Glatzel 1993). The higher value of $L / M$ adopted here amplifies the tendency towards instability through shorter thermal timescales. 

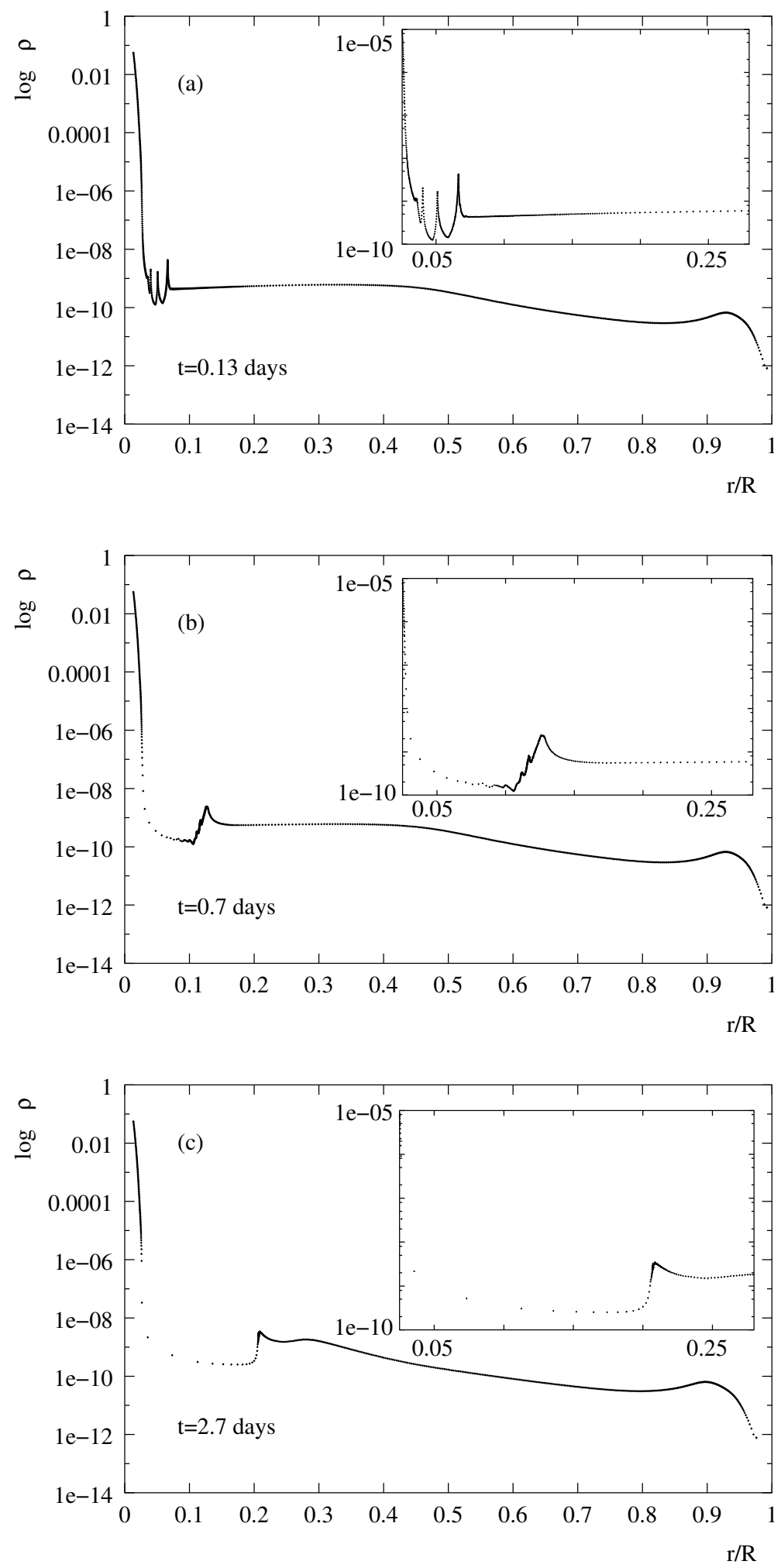

Figure 8.2: Snapshots of the density $\rho$ as a function of relative radius $r / R$ after 0.13 (a), 0.7 (b) and 2.7 (c) days of simulated time for an envelope model of the star P Cygni. Details of the instability region are shown in the small panels. The grid resolution is indicated by dots. 
Table 8.1: Results of a linear non-adiabatic stability analysis for a model of the star $\mathrm{P}$ Cygni. Frequencies $\omega_{R}$ and growth rates $\omega_{I}$ of some representative high and low order unstable strange modes are given in units of $\left[10^{-5} \mathrm{sec}^{-1}\right]$. Negative $\omega_{I}$ corresponds to instability.

\begin{tabular}{cccccc}
\hline$\omega_{R}$ & 0.378 & 0.878 & 0.946 & 0.993 & 1.040 \\
$\omega_{I}$ & $-3.237 \cdot 10^{-6}$ & $-1.233 \cdot 10^{-2}$ & $-1.142 \cdot 10^{-3}$ & $-8.911 \cdot 10^{-3}$ & $-9.012 \cdot 10^{-3}$ \\
\hline$\omega_{R}$ & 1.087 & 1.203 & 1.296 & 1.389 & 21.33 \\
$\omega_{I}$ & $-5.460 \cdot 10^{-3}$ & $-3.437 \cdot 10^{-3}$ & $-5.711 \cdot 10^{-3}$ & $-1.969 \cdot 10^{-3}$ & -3.149 \\
\hline$\omega_{R}$ & 25.05 & 38.88 & 44.18 & 46.42 & 49.55 \\
$\omega_{I}$ & -3.890 & -7.806 & -8.428 & -7.154 & -8.721 \\
\hline
\end{tabular}

We have performed a linear stability analysis of the model using the Riccati method (Gautschy \& Glatzel 1990) and identified various unstable modes. The results of the analysis are summarised in Table 8.3.1, where eigenfrequencies of some low and high order unstable strange modes are given. The Lagrangian displacement component of a high order eigenfunction is shown as a function of relative radius in Figure 8.1.a. It is oscillatory and confined between $0.05<r / R<0.12$, at the edge of the stellar core and close to the opacity peak around $\log T \approx 5.3$. (In this region inversions of both the sound speed and the density do occur.) The corresponding work integral is given in Figure 8.1.b. It indicates the region which drives the pulsation and is responsible for the instability. (For a discussion of the applicability of the concept of the work integral in the case of highly non-adiabatic pulsations see Glatzel (1994).) Although this region coincides with the opacity maximum, the mechanism driving the instability cannot be the classical $\kappa$-mechanism, since the modes are unstable also in the NAR-limit (see, e.g., Gautschy \& Glatzel 1990), which implies vanishing specific heat. Rather, this is a strange mode instability, caused by the low ratio of gas pressure to total pressure $\beta$, which is found for this model in the vicinity of the opacity peak.

Using the numerical procedure described in Section 7.2 we have investigated the nonlinear evolution of instabilities of this model, which is dominated by high order modes due to their extremely large growth rates $\omega_{I}$. Figure 8.2 shows snapshots of the density $\rho$ as a function of relative radius $r / R$ after $0.13,0.7$ and 2.7 days of simulated time. ( $R$ is the initial radius of the model.) The instability generates sound waves at $r / R \approx 0.05$ thus forming a sequence of shocks (Figure 8.2.a), which then travel outwards, thereby transferring mass into the outer parts of the envelope. Owing to the Lagrangian approach chosen here, this leads to a decreased grid resolution as can be seen in Figures 8.2.b and 8.2.c. Subsequent unstable sound waves appearing at $r / R \approx 0.05$ can then not be resolved any longer. As a consequence the instability is suppressed and the mass flow out of the instability domain ceases. This fact would be crucial should the instability, as suspected, lead to an outburst of and mass loss from the object.

The simulation of the non linear evolution of instabilities in stellar envelopes requires 
an adequate resolution of shocks, ionisation zones and instability regions. For this purpose, various approaches have been proposed to overcome resolution problems (see, e.g., the adaptive grid algorithms used by Stellingwerf (1975) and Dorfi \& Feuchtinger (1991). With respect to shocks and zones exhibiting large gradients of the density, the resolution problem is solved intrinsically by the Lagrangian description adopted here. However, a sufficient resolution of the instability region driving the pulsation and mass flow is not guaranteed by the Lagrangian and any of the other mentioned approaches. A systematic study of the influence of grid resolution on the non-linear evolution of strange mode instabilities will therefore be given in the following section.

\subsubsection{The influence of resolution}

The influence of grid resolution on the simulation of dynamical instabilities in stellar envelopes is studied on an envelope model having the parameters $M=45 M_{\odot}, T_{\text {eff }}=$ $33890 \mathrm{~K}, L=537 \cdot 10^{3} L_{\odot}$ and the chemical composition $X=0.7, Y=0.28$ and $Z=0.02$. It has been chosen for the resolution study for two reasons. Firstly, the linear non-adiabatic stability analysis provides only one unstable mode with frequency $\omega_{R}=1.99 \cdot 10^{-5} \mathrm{sec}^{-1}$ and growth rate $\omega_{I}=3.98 \cdot 10^{-7} \mathrm{sec}^{-1}$ and this facilitates the comparison with the non-linear simulation and its validation. Secondly, this model has been studied previously by Dorfi \& Gautschy (2000) and been denoted M45. Thus our results can be compared directly to those obtained with the Vienna-RHD code. Dorfi \& Gautschy (2000) found, that M45 reaches a stable limit cycle and pulsates regularly with pulsation amplitudes reaching 15 per cent of the escape velocity at the stellar surface. As Dorfi \& Gautschy (2000) we will refer to it as M45 in the following.

Adopting the parameters of M45, three configurations with a resolution of 256, 512 and 1024 gridpoints were constructed, which will be referred to as R256, R512 and $\mathrm{R} 1024$, respectively. The results of the simulations are summarised in Table 8.3.2, where the growth rates $\omega_{I}$, the frequencies $\omega_{R}$, the maximum velocities normalised to the escape velocity, the grid resolution (total number of gridpoints) and the resolution of the instability zone (number of gridpoints there) between $T=1 \cdot 10^{5} \mathrm{~K}$ and $=3.1 \cdot 10^{5}$ $\mathrm{K}$ are presented. For comparison, the results of the independent, linear, non-adiabatic stability analysis (LNA) and the results obtained by Dorfi \& Gautschy (2000) are also given there. The LNA was performed using the Riccati method (see Gautschy \& Glatzel 1990).

The results of the non-linear simulations are shown in Figure 8.3 for the models R256 (first row), R512 (second row) and R1024 (third row). The absolute value of the velocity $|v|$ as a function of time at the outermost gridpoint is given on a logarithmic scale for the entire integration interval in the first column of Figure 8.3, the velocity $v$ for two pulsation periods in the non-linear regime in the second column. The third column contains the corresponding periodograms, i.e., the power spectral density as a function of frequency.

The power spectral density (PSD) was calculated from the time series of the velocity $v$ starting at the time $t$ when the pulsation amplitude has saturated, i.e., the linear regime 
Table 8.2: Growth rates $\omega_{I}$, frequencies $\omega_{R}$, the maximum velocities normalised to the escape velocity, total number of gridpoints and number of gridpoints in the instability region between $T=1 \cdot 10^{5} \mathrm{~K}$ and $T=3.1 \cdot 10^{5} \mathrm{~K}$ for various models. For comparison, the results of an independent, linear, non-adiabatic stability analysis (LNA) and the results obtained by Dorfi \& Gautschy (2000) (M45) are also given.

\begin{tabular}{lccccc} 
Model & $\omega_{R}\left[\mathrm{sec}^{-1}\right]$ & $\omega_{I}\left[\mathrm{sec}^{-1}\right]$ & $v_{\max } / v_{\mathrm{esc}}$ & Grid Resolution & $\begin{array}{c}\text { Resolution of } \\
\text { instab. region }\end{array}$ \\
\hline LNA & $1.99 \cdot 10^{-5}$ & $3.98 \cdot 10^{-7}$ & - & - & - \\
M45 & $2.01 \cdot 10^{-5}$ & - & 0.15 & 500 & - \\
\hline R256 & $1.98 \cdot 10^{-5}$ & $2.73 \cdot 10^{-7}$ & 0.09 & 256 & 84 \\
R512 & $1.97 \cdot 10^{-5}$ & $3.45 \cdot 10^{-7}$ & 0.15 & 512 & 160 \\
R1024 & $1.98 \cdot 10^{-5}$ & $3.94 \cdot 10^{-7}$ & 0.18 & 1024 & 350 \\
R512V5 & $1.99 \cdot 10^{-5}$ & $3.34 \cdot 10^{-7}$ & 0.21 & 512 & 160 \\
R512V50 & $1.99 \cdot 10^{-5}$ & $3.34 \cdot 10^{-7}$ & 0.19 & 512 & 160 \\
R512V250 & $1.98 \cdot 10^{-5}$ & $3.32 \cdot 10^{-7}$ & 0.15 & 512 & 160 \\
R512V1000 & $1.97 \cdot 10^{-5}$ & $3.34 \cdot 10^{-7}$ & 0.16 & 512 & 160 \\
\hline C512 & $1.98 \cdot 10^{-5}$ & $3.94 \cdot 10^{-7}$ & 0.16 & $512 / 672$ & $160 / 320$
\end{tabular}

of exponential growth of the time series at the beginning of the simulation has been discarded. This corresponds to $t \approx 8.5 \cdot 10^{6} \mathrm{sec}, t \approx 6.5 \cdot 10^{6} \mathrm{sec}$ and $t \approx 5.8 \cdot 10^{6} \mathrm{sec}$ for the models R256, R512 and R1024, respectively. For the Fourier transform an equidistantly sampled time series was obtained by interpolation. Before calculating the periodogram, the data was weighted with a triangular window. To achieve a higher frequency resolution, oversampling was used while calculating the discrete Fourier transform. The width of the peaks depends on the number of sampled periods.

In the first column of Figure 8.3, the exponential growth of the velocity $v$ in the linear regime, when the physical instability has overcome the level of numerical perturbations $\left[\mathcal{O}\left(10^{3} \mathrm{~cm} \mathrm{sec}^{-1}\right)\right]$, is clearly visible for all considered models. The corresponding growth rates have been calculated from the slope of the increase of the velocity $v$ and are indicated by the solid lines. The extrapolation of these lines to $t=0$ indicate that in all cases the pulsations commence from a contribution of the order of a few cm sec $\mathrm{in}^{-1}$ the unstable mode to the initial numerical noise. Comparing these growth rates with that of the independent linear, non-adiabatic stability analysis, they are lower by $\sim 31,13$ and one per cent for the models R256, R512 and R1024, respectively. Furthermore, the pulsation amplitude reached increases with the grid resolution from 9 to 18 per cent of the escape velocity. Our model R512, which has a resolution comparable to M45, yields similar results as those reported in Dorfi \& Gautschy (2000).

In the second column of Figure 8.3 the final form of the velocity curve of the pulsation is shown for the considered models. More details become visible with increasing grid resolution. The most striking feature is an additional local maximum in the pulsation velocity for the model R512, which becomes even more pronounced for the model R1024. Moreover, the increasing branches of the velocity curves become steeper with increasing 

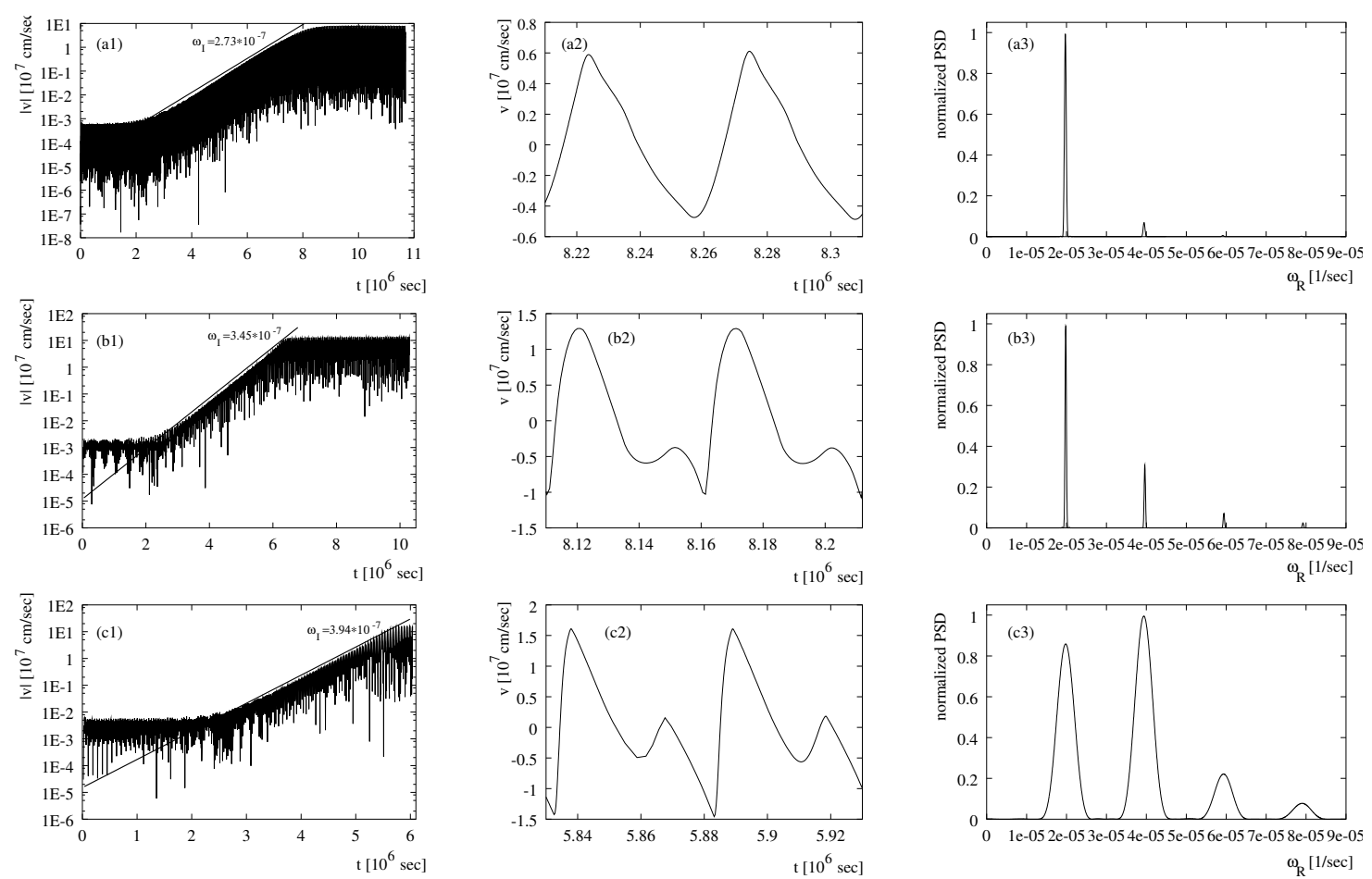

Figure 8.3: The velocity $v$ as a function of time during the entire integration interval (1) and two periods of the final pulsation (2) for the models R256 (a), R512 (b) and R1024 (c). The corresponding periodograms are given in column (3).

resolution.

This behaviour may also be read off from the periodograms given in the third column. Whereas for the model R256 the main contribution to the pulsation is the fundamental mode, the contribution of the higher overtones increases with resolution. For the model R1024 the first overtone contributes even more than the fundamental mode. Note, that the width of the various peaks depends on the number of sampled periods, which was smaller for R1024 due to the higher computational effort. The period of the fundamental mode is in very good agreement with that of the LNA, differing by at most 1 per cent. The deviations from the results discussed in Dorfi \& Gautschy (2000) may be caused by the fact that these authors do not include any convective flux in their calculation.

We conclude that with respect to resolution, the most sensitive quantities are the growth rate of the instability $\omega_{I}$, the pulsation amplitude $\left(v_{\max }\right)$ and the form of the final pulsation. For the model considered, a minimum resolution of $\sim 1000$ gridpoints should be used for reliable results. As will be shown in section 8.3.5, the resolution of the instability region is primarily responsible for a correct reproduction of $\omega_{I} \cdot v_{\max }$ and the form of the pulsation depend primarily on the dissipation incorporated in the simulation, which will be demonstrated in the next section. 

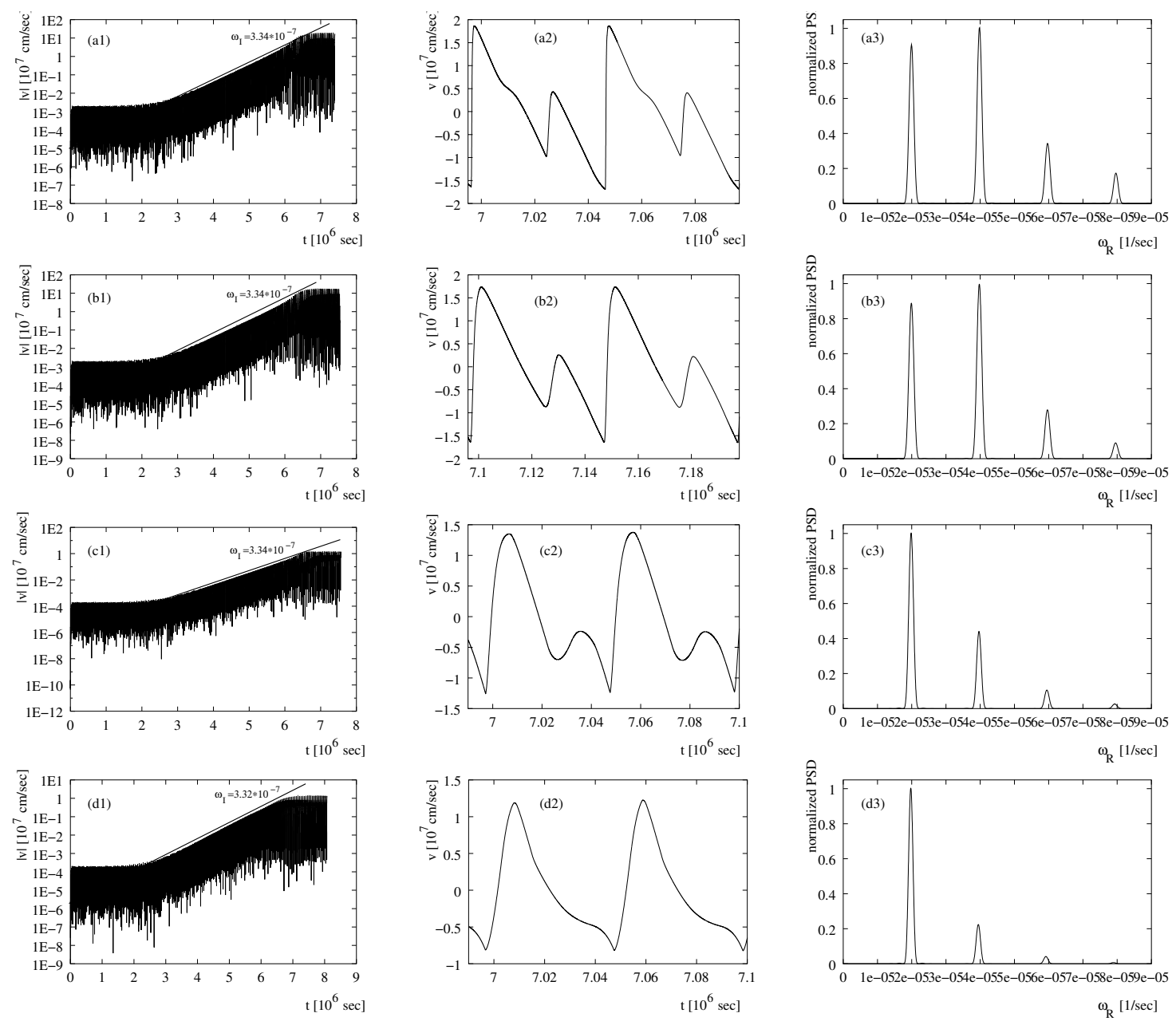

Figure 8.4: Same as Figure 8.3, but for the models R512V5 (a), R512V50 (b), R512V250 (c) and R512V1000 (d).

\subsubsection{The influence of artificial viscosity}

One of the consequences of an enhanced resolution is the reduction of the intrinsic viscosity of the difference scheme. Therefore, we have systematically investigated the influence of the artificial viscosity parameter $C_{0}$. To save computing time, the tests were carried out with a moderate grid resolution of 512 gridpoints (model R512), and $C_{0}$ was varied between 5 and 1000. The value adopted in the models R256, R512 and R1024 was 500.

The results of the calculations are shown in Figure 8.4 and summarised in Table 8.3.2. The models R512V5, R512V50, R512V250 and R512V1000 correspond to calculations with $C_{0}=5,50,250$ and 1000, respectively. The pulsation frequencies $\omega_{R}$ and the growth rates $\omega_{I}$ turned out to be largely independent of the value of the artificial viscosity. In the linear regime the influence of artificial viscosity is negligible. Therefore growth rates $\omega_{I}$, which are determined from the exponential growth of the instabilities in the 
linear regime, are not expected to depend on $C_{0}$.

The pulsation amplitudes, however, strongly depend on artificial viscosity and $v_{\max }$ varies between 15 and 21 per cent of the escape velocity. The periodograms show an increased power in the higher harmonics with decreased artificial viscosity. This is in line with the steeper gradients and a more detailed substructure of the velocity curves.

Artificial viscosity - in contrast to the resolution dependent intrinsic viscosity of the difference scheme - is unimportant in the linear regime. In the non-linear regime, both effects play a significant role concerning the pulsation amplitudes and the form of the final velocity curves. Thus, for the study of mass loss, a reduction of artificial viscosity is not sufficient and a high resolution of the instability region is necessary to guarantee reliable results. One way to achieve an appropriate resolution consists of continuously inserting gridpoints during the simulation whenever necessary. Details of such a procedure are presented in the following section.

\subsubsection{Numerical Procedure}

The insertion of new grid points during a simulation poses two problems: Firstly, the smoothness of the grid, i.e., the slow variation of the length of neighbouring grid cells, has to be guaranteed. Otherwise, the errors when calculating grid derivatives can cause unphysical oscillations of the solution. Secondly, the definition of the variables velocity, temperature, internal energy, etc. on the new gridpoints has to be chosen such, that equations 8.7-8.10 are satisfied, which is not guaranteed by interpolation. As for the relaxation of initial models, small errors in these equations can lead to large artificial oscillations, which is most pronounced in the almost hydrostatic inner regions.

In the approach presented, the grid resolution is increased by inserting new nodes in the middle of the cells of the reconstruction area. Subsequently, the gridpoints at the inner and outer edges of this area are redistributed such that the smoothness of the grid is guaranteed. This yields the radii $\tilde{r}_{i}$ of the new grid. The maximum variation of the length of neighbouring cells was required to be less than 10 per cent.

To overcome the second problem, an approach similar to the relaxation procedure described in section 8.2.1 was followed. In a first step, the right hand sides of equations 8.8 and 8.9 are evaluated at the current timelevel $t_{n}$ on the old grid. Denoting them by $\phi_{v, i}$ and $\phi_{\epsilon, i}$ we obtain

$$
\phi_{v, i}=-r_{i}^{2} \cdot \frac{\left(g_{i}-g_{i-1}\right)}{\bar{m}_{i}}-\frac{4 \pi G M_{r, i}}{r_{i}^{2}}
$$

and

$$
\begin{aligned}
\phi_{\epsilon, i}= & -g_{i} \cdot \frac{\left(r_{i+1}^{2} \cdot v_{i+1}-r_{i}^{2} \cdot v_{i}\right)}{m_{i}} \\
& -\frac{r_{i+1}^{2} \cdot\left(F_{\mathrm{rad}, \mathrm{i}+1}+F_{\mathrm{con}, \mathrm{i}+1}\right)}{m_{i}} \\
& +\frac{r_{i}^{2} \cdot\left(F_{\mathrm{rad}, \mathrm{i}}+F_{\mathrm{con}, \mathrm{i}}\right)}{m_{i}}
\end{aligned}
$$


In the second step, $\phi_{v, i}, \phi_{\epsilon, i}, v_{i}$ and $F_{\text {con,i }}$ are interpolated (using monotonic, rational, cubic Hermite interpolation, see, e.g., Dougherty, Edelman \& Hyman 1989) on the new gridpoints and denoted by $\tilde{\phi}_{v, i}, \tilde{\phi}_{\epsilon, i}, \tilde{v}_{i}$ and $\tilde{F}_{\text {con,i }}$.

Temperature $\tilde{T}_{i}$ and the pressure $\tilde{p}_{i}$ on the new grid $\tilde{r}_{i}$ are then determined by the equations

$$
-\tilde{r}_{i}^{2} \cdot \frac{\left(\tilde{g}_{i}-\tilde{g}_{i-1}\right)}{\tilde{m}_{i}}-\frac{4 \pi G \tilde{M}_{\tilde{r}, i}}{\tilde{r}_{i}^{2}}=\tilde{\phi}_{v, i}
$$

and

$$
\begin{aligned}
& -\tilde{g}_{i} \cdot \frac{\left(\tilde{r}_{i+1}^{2} \cdot \tilde{v}_{i+1}-\tilde{r}_{i}^{2} \cdot \tilde{v}_{i}\right)}{\tilde{m}_{i}} \\
& -\frac{\tilde{r}_{i+1}^{2} \cdot\left(\tilde{F}_{\mathrm{rad}, \mathrm{i}+1}+\tilde{F}_{\mathrm{con}, \mathrm{i}+1}\right)}{\tilde{m}_{i}} \\
& +\frac{\tilde{r}_{i}^{2} \cdot\left(\tilde{F}_{\mathrm{rad}, \mathrm{i}}+\tilde{F}_{\mathrm{con}, \mathrm{i}}\right)}{\tilde{m}_{i}}=\tilde{\phi}_{\epsilon, i}
\end{aligned}
$$

We drop the $\sim$ for readability, use the diffusion equation for energy transport (equation 8.10) and the definition of the mass of a cell/node and are left with

$$
\begin{aligned}
\frac{1}{2}\left[m_{i}+\frac{1}{3} \rho_{i-1}\left(r_{i}^{3}-r_{i-1}^{3}\right)\right] & \\
\times\left[\phi_{v, i} \cdot r_{i}^{2}+4 \pi G M_{r_{i}}\right] & \\
+r_{i}^{4}\left(p_{i}-p_{i-1}\right) & =0 \\
\frac{1}{2}\left[m_{i}+\frac{1}{3} \rho_{i-1}\left(r_{i}^{3}-r_{i-1}^{3}\right)\right] & \\
\times\left[\phi_{\epsilon, i} \cdot m_{i}+p_{i}\left(r_{i+1}^{2} v_{i+1}-r_{i}^{2} v_{i}\right)\right. & \\
\left.+F_{\text {rad }, \mathrm{i}+1}+F_{\text {con }, \mathrm{i}+1}-F_{\text {con }, \mathrm{i}}\right] & \\
+r_{i}^{4} \cdot \bar{\theta}_{i}\left(T_{i}-T_{i-1}\right) & =0
\end{aligned}
$$

This is an initial value problem, since $T_{i-1}$ and $p_{i-1}$ are completely determined by the corresponding values at nodes $i$ and $i+1$ and in cells $i$. Together with the equation of state for the density (equation 8.14), the system can be solved iteratively, e.g., by a standard Newton method.

For the next integration step the density $\rho_{i-1}$ (via the equation of state), the mass $m_{i-1}$ and the radiative flux $F_{\text {rad,i }}$ (via the diffusion equation for energy transport) are then calculated using $T_{i-1}$ and $p_{i-1}$.

We note, that the procedure presented violates the conservativity otherwise inherent in the numerical scheme, since the interpolation errors in $\tilde{\phi}_{v, i}, \tilde{\phi}_{\epsilon, i}, \tilde{v}_{i}$ and $\tilde{F}_{\text {con,i }}$ are retained. The relative errors in mass, momentum and energy conservation thus generated depend on the region where grid reconstruction is applied and amount to $\sim 10^{-4}$ for the inner and $\sim 10^{-7}$ for the outer envelope. A more detailed study of the consequences of conservativity violation by the grid reconstruction is presented in the following section. 

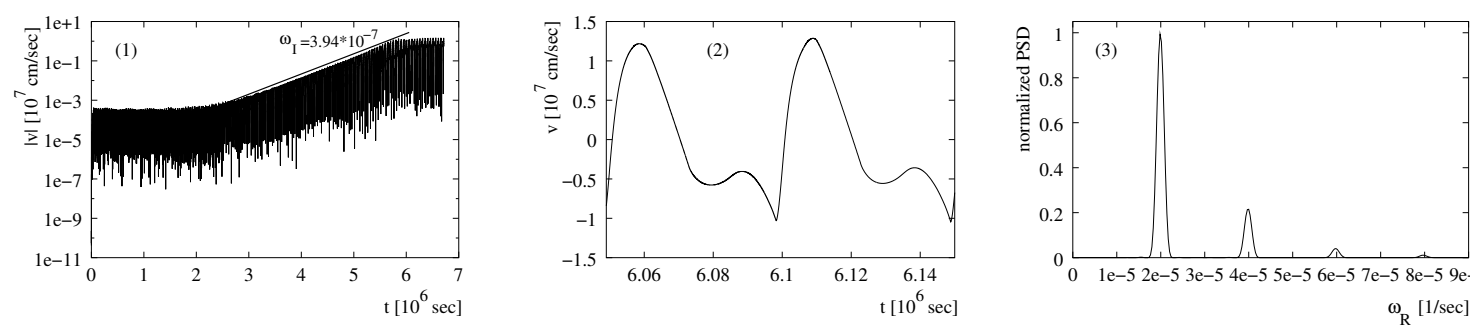

Figure 8.5: Same as Figure 8.3, but for the model C512.

\subsubsection{Validation of the grid reconstruction procedure}

To study the influence of the proposed grid reconstruction procedure in long term simulations, the following test was carried out: On the basis of model R512, the resolution of the instability region around $\log T \approx 5.3$ was switched between 160 and 320 gridpoints every 100 timesteps using the procedure discussed above. We will refer to this test as model C512 in the following.

The test calculation covers $\sim 6.8 \cdot 10^{6} \mathrm{sec}$ and consists of $\sim 200000$ integration timesteps, corresponding to 2000 grid reconstructions. The results of the simulation are shown in Figure 8.5 and are summarised in Table 8.3.2. C512 excellently reproduces the growth rate $\omega_{I}$, as can be read off from Figure 8.5.a1. This is an immediate consequence of the increased resolution of the instability region. As expected, the pulsation amplitude $\left(v_{\max }\right)$ lies between those of models R512 and R1024 and is thus consistent with the resolution study in section 8.3.2. The final form of the pulsation, shown in Figure 8.5.a2, does not differ significantly from that obtained for the model R512 (compare Figure 8.3.b2). This is confirmed by the periodogram in Figure 8.5.a3, which exhibits only a slightly smaller contribution of the first overtone compared with Figure 8.3.b3. We thus conclude that violation of conservativity and dissipation caused by the grid reconstruction has a negligible influence on the results of the calculation.

The final relative error in the mass of the stellar envelope was found to be 1 per cent. It is made up of the errors of the individual reconstructions, which for C512 are of the order of $\sim 10^{-4}$. Usually, grid reconstruction in the instability region will become necessary only a few times for a complete simulation, thus reducing the final error to at most $10^{-3}$.

\subsection{Conclusions}

The study of the non-linear evolution of strange mode instabilities in a model of the star P Cygni turned out to suffer from an insufficient resolution of the instability region. This is due to a Lagrangian description together with a mass flow out of the instability domain caused by the instability itself. As a consequence of the insufficient resolution and an increased dissipation in the driving regions of the stellar envelope, the instability is suppressed and the mass flow out of the instability domain ceases. This fact would be crucial should the instability, as suspected, lead to an outburst of and mass loss from 
the object. Thus the development of a grid reconstruction algorithm was necessary to guarantee a sufficient resolution.

Reconstruction of the grid, in particular inserting new grid points, can cause artificial oscillations. To avoid them, the grid reconstruction procedure presented here consists of two steps: Firstly, new gridpoints are inserted into the reconstruction domain and the adjacent regions, such that the grid remains smooth. Then the stellar envelope is reintegrated on the reconstructed grid with prescribed velocity field $v(r)$ and convective flux $F_{\text {con }}(r)$. The latter are derived from their values on the previous grid by interpolation. This approach does not suffer from unphysical oscillations.

By comparison to results obtained without grid reconstruction the procedure was tested and validated. The solution does not become affected by additional unphysical features and the effect of the approach may be explained in terms of modified resolution. Although violation of conservativity did not imply any severe consequences, it would still be preferable to maintain the conservativity otherwise inherent in the numerical scheme. An attempt in this direction might consist of using the degrees of freedom in the interpolation step of the grid reconstruction procedure to achieve conservativity. Whether a modified functional minimisation approach as proposed by Chernigovski \& Novac (1996) can provide values for the variables, which at least minimise the errors in the conserved quantities, remains to be seen.

The presented method might also be incorporated in the rezoning step of an arbitrary Lagrangian Eulerian (ALE) technique to guarantee numerical stability and to avoid artificial oscillations (for an introduction to ALE see, e.g., Hirt, Amsden \& Cook 1997). In this method, the procedure of filling-in new gridpoints is replaced by moving the grid according to the special requirements, which consist of properly resolving the instability region. An occasional remapping of the nodes of the grid to their original positions may be a suitable prescription for the grid motion.

\section{Acknowledgements}

We thank Professors K.J. Fricke and G. Warnecke for encouragement and support. Financial support by the Graduiertenkolleg "Strömungsinstabilitäten und Turbulenz" (MG) and by the DFG under grant WA 633 12-1 (SC) is gratefully acknowledged. This work has gained from Jörg Hubers expertise on signal analysis and we are indebted to him for calculating the presented periodograms. The numerical computations have been carried out using the facilities of the GWDG at Göttingen. 


\section{Bibliography}

Baker N.H., Kippenhahn R., 1965, ApJ, 142, 869

Chernigovski S., Novac S., 1996, in Stancu D.D., Coman G., Breckner W.W., BlagaP., eds, Proc. ICAOR, Transilvania Press, vol.2, p. 47

Cox J.P., 1980, Theory of Stellar Pulsation. Princeton Univ. Press, Princeton, NJ

Dougherty R.L., Edelman A., Hyman J.M., 1989, Math. of Comp. 52, 186, 471

Dorfi E. A., 1998, Computational Methods for Astrophysical Fluid Flow, ed. O. Steiner \& A. Gautschy (Berlin: Springer), 263

Dorfi E. A., Drury L.O'C., 1987, J. Comput. Phys., 69, 175

Dorfi E. A., Feuchtinger M. U., 1995, Comput. Phys. Commun., 89, 69

Dorfi E. A., Feuchtinger M. U., 1991, A\&A, 249, 417

Dorfi E. A., Gautschy A., 2000, ApJ, 545, 982

El Eid M.F., Hartmann D., 1993, ApJ, 404, 271

Fraley, G.S., 1968, Ap\&SS, 2, 96

Gautschy A., Glatzel W., 1990, MNRAS, 245, 154

van Genderen, 2001, A\&A, 366, 508

Glatzel W., 1994, MNRAS, 271, 66

Glatzel W., Kiriakidis M. 1993, MNRAS, 263, 375

Glatzel W., Kiriakidis M., Chernigovskij S., Fricke K.J. 1999, MNRAS, 303, 116

Hirt C.W., Amsden A.A., Cook J.L., 1997, Journal of Comp. Phys., 135, 108

Humphreys R.M., Davidson K., 1979, ApJ, 232, 409

Humphreys R.M., Davidson K., 1994, PASP, 106, 704

Iglesias C.A., Rogers F.J., Wilson B.G., 1992, ApJ, 397, 717

Kiriakidis M., Fricke K.J., Glatzel W., 1993, MNRAS, 264, 50

Najarro F., Hillier D.J., Stahl O., 1997, A\&A, 326, 1117

Pauldrach A.W.A., Puls J., 1990, A\&A, 237, 409

Rogers F.J., Iglesias C.A., 1992, ApJS, 79, 507

Samarskii A., Popov Yu., 1969, Zh. Vychisl. Mat. i Mat. Fiz., 9, 953 Diff. Eqns., 17, 854

Stellingwerf R.F., 1975, ApJ, 195, 441 


\section{Summary}

\subsection{Summary of results}

In this study we have followed the evolution of strange mode instabilities in massive stars into the non-linear regime. We have constructed stellar models which in the HR diagram are situated in the instability region previously identified by Kiriakidis et al. (1993). As expected, the models turned out to suffer from multiple strange mode instabilities.

The evolution of the instabilities was then followed into the non-linear regime by direct numerical simulations. As an effect of the instability, sound waves are generated in the inner envelope, which travel outwards and steepen to form shock waves, which accelerate the outer mass shells and successively inflate the envelope. The instabilities act on the dynamical timescale, which for the objects studied corresponds to a few days. In this process, the outer mass shells of the envelope reach $\sim 50$ per cent of the escape velocity. Contrary to the results obtained by Glatzel et al. (1999) for hot massive stars, no pulsationally driven mass loss was found for the considered cool objects investigated here. A previously unknown phenomenon consists of capturing of shock waves in the hydrogen ionisation zone. The captured shock then starts to oscillate on timescales much shorter than the dynamical one. For the models studied by Glatzel et al. (1999), hydrogen is always completely ionised due to their high effective temperature. A detailed summary of the physics of shock oscillations, their appropriate numerical treatment and the grid reconstruction procedure is presented in the following subsections.

\subsubsection{Paper I: Captured shocks}

As a first step, the origin of the shock oscillations observed in numerical simulations had to be clarified. In principle, the observed rapid oscillations could have been numerical artifacts. The only independent way to distinguish between physical and numerical instability is a linear stability analysis, which was therefore applied to the stellar envelope containing a captured shock. The analysis differed from the standard linear stability analysis as described by, e.g., Baker and Kippenhahn (1962), because the non-vanishing background flow had to be taken into account. As a result of the analysis, unstable modes with appropriate frequencies and high growth rates were identified. These are localised in the shock zone and excited there. Thus we have proven that the shock oscillations are of physical origin. 
In a second step, a simplified analytical model of the envelope containing a captured shock was constructed to clarify which physical parameters are responsible for the instability. As a result, the shock was shown to split the envelope into three quasi-independent acoustic cavities, each of them providing a separate spectrum of eigenmodes. Thus, a detailed study of the origin of the instability could be restricted to the zone containing the captured shock. On the basis of this shock zone model, we could show that the stratification of the envelope, in particular the temperature gradient, is responsible for the instability.

\subsubsection{Paper II: Domain decomposition}

Since the captured shock oscillates on timescales much shorter than the dynamical one, the integration timestep is strongly limited. This inhibits the long term study of the system. Therefore, having proven the shock oscillations to be of physical, rather than numerical origin, an adequate numerical treatment of the problem had to be developed. It takes advantage of the fact that the timescales present are vastly different.

The procedure consists of the following steps: We first divide the domain of computation into three subdomains, according to the different time variation of dependent variables in these regions. Thus we obtain a rapidly varying shock region, and, with respect to these rapid changes, quasi hydrostatic domains above and below the captured shock. All domains are then advanced in time separately according to their intrinsic timescales. For the time integration, boundary conditions had to be prescribed at the domain interfaces. The quality of the results strongly depends on the definition of proper boundary values. The different computational domains overlap. After advancing all domains by the same time $\tau$ they are reassembled such that the flawed values of the overlapping grids are discarded. Thus the errors introduced by the artificial inner boundaries are minimised. Although the procedure is not conservative, the influence of the violation of conservativity on the results were shown to be small.

Using the procedure developed, the computing time can be reduced by a factor of $\sim$ 2.5 for the models considered. The speedup mainly depends on the size of the rapidly varying domain.

\subsubsection{Paper III: Grid reconstruction}

Another effect of the instabilities consists of a mass transfer from the instability region of the stellar envelope to its outer parts. In the Lagrangian description adopted here this leads to a loss of gridpoints and resolution in the instability region. As a consequence, the driving instability is suppressed and the mass flow out of the instability domain ceases. To overcome this numerical problem, a grid reconstruction algorithm has been developed.

Reconstruction of the grid, in particular inserting new grid points, can cause artificial oscillations. To avoid them, the grid reconstruction procedure developed consists of two 
steps: Firstly, new gridpoints are inserted into the reconstruction domain and the adjacent regions, such that the grid remains smooth. Then the stellar envelope is reintegrated on the reconstructed grid with prescribed velocity field and convective flux. The latter are derived from their values on the previous grid by interpolation. This approach does not suffer from unphysical oscillations. The procedure violates the conservativity otherwise inherent in the applied difference scheme. However, this effect was found to be negligible for the models considered.

The influence of grid resolution on the simulation of the non-linear evolution of strange mode instabilities has been studied systematically. The pulsation amplitudes reached, as well as the final form of the pulsation, were found to depend sensitively on resolution, e.g., an insufficient number of gridpoints in the instability region can reduce the pulsation amplitudes by a factor of 2 .

\subsection{Conclusions}

Stellar models situated in the instability region of the HR diagram identified by Kiriakidis et al. (1993) pulsate with large amplitudes and the outer envelope reaches velocities comparable with the escape velocity. However, from the present study, we cannot confirm pulsationally driven mass loss, as reported by Glatzel et al. (1999). The discrepancy might be due to the formation of a captured, rapidly oscillating shock in the cool models considered here. It appears to have a damping influence on the primary instability. Captured shocks are not present in the models studied by Glatzel et al. (1999), since hydrogen is always completely ionised there.

As our models are restricted to the optically thick regime, line driving of mass loss is disregarded a priori. Whether a corresponding acceleration in the optically thin atmosphere could provide the missing link to the observed mass loss remains to be seen. In any case do the high pulsation velocities provide further evidence for a connection between strange mode instabilities and the observed HD limit (Humphreys \& Davidson 1979). Another strong indication for a relation between strange mode instabilities and the LBV phenomenon is the mass flow out of the instability region found in models for P Cygni. This causes numerical difficulties which still prevent a further simulation, although they have been partially solved in the present investigation.

The luminosity variations induced by the captured shocks remind of the microvariations observed for certain LBVs (as, e.g., in P Cygni (de Groot, Sterken \& van Genderen 2001)). We emphasise, however, that the one dimensional calculations presented here have to be interpreted with caution, since massive stars are known to suffer also from non-radial instabilities (Glatzel \& Mehren 1996). Whether the captured shocks survive the deformations induced by non-radial instabilities or become unstable themselves with respect to non-radial perturbations, remains to be tested by at least two dimensional calculations. They might break apart and could then contribute to the entire variation by stochastically adding high-frequency perturbations to the cyclic perturbations on the dynamical timescale induced by strange mode instabilities. 


\subsection{Future research}

Future research concerning the evolution of instabilities in stellar envelopes should include the refinement of numerical techniques. A step in this direction could be the use of arbitrary Lagrangian Eulerian (ALE) techniques (for an introduction to ALE see, e.g., Hirt, Amsden \& Cook 1997). These combine a Lagrangian description with a motion of the computational grid, i.e., the time integration is split into different phases. In a first step the considered equations are integrated in time explicitly or implicitly using a Lagrangian description. The second step consists of a remapping of the nodes of the grid (rezoning phase). The grid reconstruction procedure presented here might be incorporated in the rezoning phase of such a method to guarantee numerical stability and to avoid artificial oscillations. The procedure proposed here for the definition of the new grid could then be replaced by moving the grid according to the requirement of properly resolving the instability region. An occasional remapping of the nodes of the grid to their original positions may be a suitable prescription for the grid motion.

Although violation of conservativity of the grid reconstruction procedure did not imply any severe consequences, it would still be preferable to maintain the conservativity otherwise inherent in the numerical scheme. An attempt in this direction might consist of using the degrees of freedom in the interpolation step of the procedure to achieve conservativity. Whether a modified functional minimisation approach as proposed by Chernigovski \& Novac (1996) can provide values for the variables, which at least minimise the errors in the conserved quantities, remains to be seen.

As mentioned above, a physical effect that should be included in the calculations is the line driving in the optically thin regime. Due to the Doppler shift the velocity field induced by the primary instability yields an enhanced absorption of and acceleration by radiation. Whether this effect can be modelled by suitably adjusting the Rosseland mean of the opacity remains to be investigated.

Finally, as the growth rates of non-radial instabilities are of the same order as those for radial perturbations (Glatzel \& Mehren 1996; Glatzel \& Kaltschmidt 2002), multidimensional simulations should be carried out. We expect that they will lead to pulsation velocities comparable to the radial case. Whether the combination of radial and nonradial instabilities eventually leads to pulsationally driven mass loss remains to be seen. First progress in the direction of the development of an appropriate method for multidimensional simulations has already been made (Chernigovski, Glatzel, \& Fricke 2000). 


\section{Bibliography}

Ardelyan N., Gushchin I., 1982, Moscow Univ. Comp. Math. and Cybernetics, 3, 3

Appenzeller I., 1970, A\&A, 5, 355

Appenzeller I., 1988, in IAU Colloquium, Physics of Luminous Blue Variables, ed. K.

Davidson et. al., Kluwer Acad. Press, 113, 195

Baker N.H., Kippenhahn R., 1962, Z. f. Astrophysik, 54, 114

Baker N.H., Kippenhahn R., 1965, ApJ, 142, 869

Böhm-Vitense E., Z. f. Astrophysik, 1958, , 46, 108

Buchler J.R., Whalen P., 1990, in The Numerical Modelling of Nonlinear Stellar

Pulsations Problems and Prospects, ed. J. R. Buchler, NATO ASI ser. C302, Kluver

Academic Publishers, Dordrecht, 315

Cairns R.A., 1979, Journal of Fluid Mech., 92, 1

Castor J.I., 1971, ApJ, 166, 109

Chernigovski, S., Glatzel, W., Fricke, K.J., 2000, Astronomische Gesellschaft Meeting Abstracts, 17, 23

Chernigovski S., Grott M., Glatzel W., 2003, MNRAS, submitted

Chernigovski S., Novac S., 1996, in Stancu D.D., Coman G., Breckner W.W., Blaga P., eds, Proc. ICAOR, Transilvania Press, vol.2, p. 47

Chen L., 1987, Waves and Instabilities in Plasmas, World Scientific, Lecture Notes in Physics Vol. 12, Singapore

Christy R.F., 1966, in Stein R.F., Cameron A.G.W., ed., Stellar Evolution. Plenum Press, New York, p 359

Cox J.P., 1980, Theory of Stellar Pulsation. Princeton Univ. Press, Princeton, NJ

Davey A., 1977, J. Comput. Phys., 24, 331

Davidson K., 1988, in IAU Colloquium, Physics of Luminous Blue Variables, ed. K.

Davidson et. al., Kluwer Acad. Press, 113, 101

De Jager C., 1984, A\&A, 138, 252

Dougherty R.L., Edelman A., Hyman J.M., 1989, Math. of Comp. 52, 186, 471

Dorfi E. A., 1998, Computational Methods for Astrophysical Fluid Flow, ed. O. Steiner \& A. Gautschy (Berlin: Springer), 263

Dorfi E. A., Drury L.O'C., 1987, J. Comput. Phys., 69, 175

Dorfi E. A., Feuchtinger M. U., 1991, A\&A, 249, 417

Dorfi E. A., Feuchtinger M. U., 1995, Comput. Phys. Commun., 89, 69

Dorfi E. A., Gautschy A., 2000, ApJ, 545, 982 
El Eid M.F., Hartmann D., 1993, ApJ, 404, 271

Fraley G.S., 1968, Ap\&SS, 2, 96

Gautschy A., Glatzel W., 1990a, MNRAS, 245, 154

Gautschy A., Glatzel W., 1990b, MNRAS, 245, 597

Gautschy A., 1993, MNRAS, 265, 340

van Genderen A.M., 2001, A\&A, 366, 508

Glatzel W., 1987, MNRAS, 228, 77

Glatzel W., Gautschy A., 1992, MNRAS, 256, 209

Glatzel W., 1994, MNRAS, 271, 66

Glatzel W., 1998, A\&A, 339, L5

Glatzel W., Kaltschmidt H.-O., 2002, MNRAS, 337, 743

Glatzel W., Kiriakidis M., 1993a, MNRAS, 262, 85

Glatzel W., Kiriakidis M., 1993b, MNRAS, 263, 375

Glatzel W., Kiriakidis M., 1998, MNRAS, 295, 251

Glatzel W., Kiriakidis M., Fricke K.J., 1993, MNRAS, 262, L7

Glatzel W., Kiriakidis M., Chernigovskij S., Fricke K.J., 1999, MNRAS, 303, 116

Glatzel W., Mehren S., 1996, MNRAS, 282, 1470

de Groot M., Sterken C., van Genderen A.M., 2001, A\&A, 376,224

Grott M., Glatzel W., Chernigovski S., 2003a, MNRAS, submitted

Grott M., Chernigovski S., Glatzel W., 2003b, MNRAS, accepted

Hansen C.J., Aizenman M.L., Ross R.R., 1976, ApJ, 207, 736

Hirt C.W., Amsden A.A., Cook J.L., 1997, Journal of Comp. Phys., 135, 108

Humphreys R.M., Davidson K., 1979, ApJ, 232, 409

Humphreys R.M., Davidson K., 1994, PASP, 106, 704

Iglesias C.A., Rogers F.J., Wilson B.G., 1992, ApJ, 397, 717

Kippenhahn R., 1977, A\&A, 58, 267

Kippenhahn R., Weigert A., 1990, Stellar Structure and Evolution, Springer Verlag, Berlin, Heidelberg, New York

Kiriakidis M., 1992, PhD thesis, Göttingen (in german)

Kiriakidis M., Fricke K.J., Glatzel W. 1993, MNRAS, 264, 50

Kiriakidis M., Glatzel W., Fricke K.J., 1996, MNRAS, 281, 406

Langer N., 1998, A\&A, 329, 551

Langer N., 1999, ApJ, 520, L49

Ledoux P., 1941, ApJ, 94, 537

Ledoux P., 1945, ApJ, 102, 143

Maeder A., 1988, in IAU Colloquium, Physics of Luminous Blue Variables, ed. K.

Davidson et. al., Kluwer Acad. Press, 113, 18

Maeder A., 1992, in Instabilities in Evolved Super- and Hypergiants, ed. C. de Jager and H. Nieuwenhuijzen, Amsterdam, North Holland, 138

Moskalik P., Dziembowski W.A., 1992, A \& A, 256, L5

Najarro F., Hillier D.J., Stahl O., 1997, A\&A, 326, 1117

Noh W.F., 1987, J. Comput. Phys., 72, 78

Osaki Y., 1975, PASJ, 44, 27 
Pauldrach A.W.A., Puls J., 1990, A\&A, 237, 409

Press H., Teukolski S.A., Vetterling W.T., Flannery B.P., 1992, Numerical recipies in Fortran, second edition, Cambridge Univ. Press

Rogers F.J., Iglesias C.A., 1992, ApJS, 79, 507

Samarskii A., Tishkin V., Favorskii A., Shashkov M., 1981, Diff. Eqns., 17, 854

Samarskii A., Popov Yu., 1969, Zh. Vychisl. Mat. i Mat. Fiz., 9, 953

Schwarzschild M., Harm R., 1959, ApJ, 191, 693

Scott M.R., 1973, J. Comput. Phys., 12, 334

Shashkov M., 1996, Conservative Finite-Difference Methods on General Grids, CRC Press, New York

Shibahashi H., Osaki Y., 1981, PASJ, 33, 427

Stellingwerf R.F., 1975, ApJ, 195, 441

Stother R., Chin C.-W., 1993, ApJ, 408, L85

Stother R., Chin C.-W., 1994, ApJ, 426, L43

Stother R., 1999a, ApJ, 516, 366

Stother R., 1999b, MNRAS, 305, 365

Tassoul J.-L., 1978, Theory of Rotating stars, Princeton University Press, Princeton

Tscharnuter W. M., Winkler K.-H., 1979, Comp. Phys. Comm., 18, 171

Unno W. Osaki Y., Ando H., Saio H., Shibahashi H., 1989, Nonradial Pulsations. Univ. Tokyo Press, Tokyo

Wu Z., 1999, SIAM J. Sci. Comput., 20, 5, 1851

Wu Z., Zou H., 2000, J. Comp. Phys., 157, 2

Zalewski J., 1992, PASJ, 44, 27

von Zeipel, H., 1924, MNRAS, 84, 665

Ziebarth K., 1970, ApJ, 162, 947 


\section{Part III}

\section{Appendix}





\section{A. On a criterion for adiabatic instability}

Starting from the integral expression

$$
\sigma^{2}=-\frac{\int_{0}^{R} r^{3} \frac{\mathrm{d}}{\mathrm{d} r}\left[\left(3 \Gamma_{1}-4\right) p\right] \frac{\delta r}{r} \mathrm{~d} r}{\int_{0}^{R} r^{4} \rho \frac{\delta r}{r} \mathrm{~d} r}
$$

for the eigenvalue $\sigma^{2}$ given by Ledoux (1945) Stothers (1999b) approximates $\delta r / r$ by a Heaviside function to estimate the lowest eigenfrequency $\sigma$ of the radial acoustic spectrum. $r^{*}$ denotes the position of the discontinuity of the Heaviside function. Integration by parts of the nominator then yields

$$
\sigma^{2}=\frac{\int_{r^{*}}^{R} 3 r^{2}\left[\left(3 \Gamma_{1}-4\right) p\right] \mathrm{d} r+r^{*^{3}}\left[\left.\left(\left.3 \Gamma_{1}\right|_{r^{*}}-4\right) p\right|_{r^{*}}\right]}{\int_{r^{*}}^{R} r^{4} \rho \mathrm{d} r}
$$

Therefore, equation 3 in Stothers (1999b) should read

$$
\sigma^{2}=\frac{\left(3\left\langle\Gamma_{1}\right\rangle-4\right) \int_{r^{* 3}}^{R^{3}} p \mathrm{~d}\left(r^{3}\right)}{\int_{r^{* 3}}^{R^{3}} \frac{1}{3} r^{2} \rho \mathrm{d}\left(r^{3}\right)}+\frac{r^{* 3}\left[\left.\left(\left.3 \Gamma_{1}\right|_{r^{*}}-4\right) p\right|_{r^{*}}\right]}{\int_{r^{*} 3}^{R^{3}} \frac{1}{3} r^{2} \rho \mathrm{d}\left(r^{3}\right)}
$$

with

$$
\left\langle\Gamma_{1}\right\rangle=\frac{\int_{r^{* 3}}^{R^{3}} \Gamma_{1} p \mathrm{~d}\left(r^{3}\right)}{\int_{r^{* 3}}^{R^{3}} p \mathrm{~d}\left(r^{3}\right)}
$$

Contrary to equation 3 in Stothers (1999b), equation A.3 contains an additional indefinite surface term comparable to the integral term. The surface term does not allow equation A.3 to be interpreted as a criterion for dynamical instability. 


\section{B. The work integral}

The work integral is a widely used tool in stellar pulsation theory to determine, which regions of the stellar envelope drive or damp the pulsations. On the basis of a time average, it is only defined for quasi-periodic motions (see, e.g., Cox 1980). However, Glatzel (1994) showed that the concept can be extended to the non-periodic case by use of an ensemble average. Here we present his derivation of the work integral, supplementing the missing calculation steps.

The work integral specifies the energy per time transferred from the background to the perturbation. We consider the mechanical part of the pulsation equations (continuity and Euler equation), which in one dimension and in the Eulerian description read

$$
\begin{aligned}
& \frac{\partial \rho}{\partial t}=-\nabla(\rho v) \\
& \frac{\partial v}{\partial t}=-\frac{1}{\rho} \nabla p-\nabla \Phi
\end{aligned}
$$

The linearisation of these equations may be obtained in the Eulerian approach by applying the linearisation operator $(\tilde{})$. It commutes with $\nabla$ and $\frac{\partial}{\partial t}$ (see, e.g., Cox 1980) and we are left with

$$
\begin{aligned}
& \frac{\partial \tilde{\rho}}{\partial t}=-\nabla(\bar{\rho} \tilde{v}) \\
& \frac{\partial \tilde{v}}{\partial t}=-\frac{1}{\bar{\rho}} \nabla \tilde{p}+\frac{1}{\bar{\rho}^{2}}(\nabla \bar{p}) \tilde{\rho}-\nabla \tilde{\Phi}
\end{aligned}
$$

where $\tilde{v}, \tilde{\rho}$ and $\tilde{p}$ are the Eulerian perturbations of velocity, density and pressure, respectively. $\bar{v}, \bar{\rho}$ and $\bar{p}$ refer to the hydrostatic background model. The problem is simplified considerably in the Cowling approximation, where the perturbations of the gravitational potential are assumed to be negligible, i.e. $\tilde{\Phi} \approx 0$. In particular, the Cowling approximation is justified for a core-envelope structure of the stellar model.

In order to derive the work integral, we switch to the Lagrangian description. This is achieved by using the relation

$$
\tilde{f}=\Delta f-\xi(\nabla \bar{f})
$$


between Eulerian perturbations $\tilde{f}$ and Lagrangian perturbations $\Delta f$. For the time evolution of the perturbations we assume a dependence of the form $e^{i \omega t}$. We note that $\xi=\tilde{v} / i \omega$ and $\partial \xi / \partial t=\tilde{v}$. Application to the continuity equation yields

$$
\begin{aligned}
& \frac{\partial}{\partial t}(\Delta \rho-\xi \nabla \bar{\rho})=-\nabla(\bar{\rho} \tilde{v}) \\
\Leftrightarrow & i \omega \Delta \rho-i \omega \xi \nabla \bar{\rho}=-\nabla(\bar{\rho} \tilde{v}) \\
\Leftrightarrow & \Delta \rho-\xi \nabla \bar{\rho}=-\nabla(\bar{\rho} \xi) \\
\Leftrightarrow & \Delta \rho+\bar{\rho}(\nabla \xi)=0
\end{aligned}
$$

The Euler equation can be rewritten as

$$
\begin{gathered}
\frac{\partial^{2} \xi}{\partial t}=-\frac{1}{\bar{\rho}} \nabla(\Delta p-\xi \nabla \bar{p})+\frac{1}{\bar{\rho}^{2}} \nabla \bar{p}(\Delta \rho-\xi \nabla \bar{\rho}) \\
\Leftrightarrow \nabla(\Delta p)=-\bar{\rho} \frac{\partial^{2} \xi}{\partial t^{2}}+\left(\nabla^{2} \bar{p}-\frac{1}{\bar{\rho}}(\nabla \bar{p})(\nabla \bar{\rho})\right) \xi
\end{gathered}
$$

Multiplying B.2 with $\frac{\partial \xi}{\partial t}$ and using B.1 we obtain

$$
\begin{aligned}
& \frac{\partial}{\partial t}\left[\frac{1}{2} \bar{\rho}\left(\frac{\partial \xi}{\partial t}\right)^{2}+\left(\frac{1}{\bar{\rho}}(\nabla \bar{p})(\nabla \bar{\rho})-\nabla^{2} \bar{p}\right) \frac{1}{2} \xi^{2}\right]= \\
& -\Delta p \frac{1}{\bar{\rho}} \frac{\partial \Delta \rho}{\partial t}-\nabla\left(\frac{\partial \xi}{\partial t} \Delta p\right)
\end{aligned}
$$

Integrating B.3 over the volume of the star, and assuming that the surface term, i.e., the acoustic flux across the surface, vanishes, we are left with

$$
\begin{aligned}
& \frac{\partial}{\partial t} \int d V\left[\frac{1}{2} \bar{\rho}\left(\frac{\partial \xi}{\partial t}\right)^{2}+\left(\frac{1}{\bar{\rho}}(\nabla \bar{p})(\nabla \bar{\rho})-\nabla^{2} \bar{p}\right) \frac{1}{2} \xi^{2}\right]= \\
& \int d V\left(-\Delta p \frac{1}{\bar{\rho}} \frac{\partial \Delta \rho}{\partial t}\right)
\end{aligned}
$$

If $\Delta p(\partial \Delta \rho / \partial t)$ is a complete differential with respect to time, e.g., if the phase lag between pressure and density perturbation vanishes, i.e., for a relation of the form $\Delta p \propto$ $\Delta \rho$, equation B.4 provides a - not necessarily positive definite - conserved energy like quantity, which may be identified with the "energy" of the pulsation. The right hand side of equation B. 4 corresponds to the $p d V$ work associated with a perturbation and forms the basis of the derivation of the classical work integral.

Contrary to the classical analysis, however, we cannot interpret the work integral here as thermal energy transferred into mechanical energy. Moreover, for non-periodic motions to be considered here, the time integral over one period cannot be defined. Rather, we take an ensemble average of equation B.4, regarding $(\xi, \Delta p, \Delta \rho)$ as a previously determined eigenfunction. A proper ensemble is provided by the fact, that the eigenfunctions are determined only up to a complex factor, accounting for the initial conditions. 
The most general form of a real eigensolution of a physical quantity $Q$ is given by

$$
Q=\mathfrak{R e}\left(e^{i \omega_{r}-\omega_{i}+i \alpha} a Q^{\dagger}(r)\right)
$$

where $Q^{\dagger}$ denotes the spatial part of the eigenfunction and the ambiguity is described by the complex number $a \exp (i \alpha)$ with phase factor $\alpha \in[0,2 \pi]$ and amplitude $a>0$. The ensemble average is then given by

$$
<Q>=\frac{1}{2 \pi} \int_{0}^{2 \pi} Q d \alpha
$$

Without loss of generality we choose $a=1$ and apply the ensemble average to equation B.4:

$$
\begin{aligned}
& \frac{\partial}{\partial t}\left\{\int _ { 0 } ^ { 2 \pi } d \alpha \int d V \left[\frac{1}{2} \bar{\rho}\left(\frac{\partial}{\partial t} \Re \mathfrak{e}\left(e^{i \omega_{r} t-\omega_{i} t+i \alpha} \xi^{\dagger}\right)\right)^{2}\right.\right. \\
& \left.\left.+\left(\frac{1}{\bar{\rho}} \nabla \bar{p} \nabla \bar{\rho}-\nabla^{2} \bar{p}\right) \frac{1}{2}\left(\mathfrak{R e}\left(e^{i \omega_{r} t-\omega_{i} t+i \alpha} \xi^{\dagger}\right)\right)^{2}\right]\right\} \\
& =\int_{0}^{2 \pi} d \alpha \int d V\left[-\mathfrak{R e}\left(e^{i \omega_{r} t-\omega_{i} t+i \alpha} \Delta p^{\dagger}\right) \frac{1}{\bar{\rho}} \frac{\partial}{\partial t} \mathfrak{M e}\left(e^{i \omega_{r} t-\omega_{i} t+i \alpha} \Delta \rho^{\dagger}\right)\right] \\
& \Leftrightarrow \frac{\partial}{\partial t}\left\{\int _ { 0 } ^ { 2 \pi } d \alpha \int d V \left[\frac{1}{4} \bar{\rho}\left(\frac{\partial}{\partial t}\left(e^{i \omega_{r} t-\omega_{i} t+i \alpha} \xi^{\dagger}+e^{-i \omega_{r} t-\omega_{i} t-i \alpha} \xi^{\dagger *}\right)\right)^{2}\right.\right. \\
& \left.\left.+\left(\frac{1}{\bar{\rho}} \nabla \bar{p} \nabla \bar{\rho}-\nabla^{2} \bar{p}\right) \frac{1}{4}\left(\left(e^{i \omega_{r} t-\omega_{i} t+i \alpha} \xi^{\dagger}+e^{-i \omega_{r} t-\omega_{i} t-i \alpha} \xi^{\dagger *}\right)\right)^{2}\right]\right\} \\
& =\int_{0}^{2 \pi} d \alpha \int d V\left[-\frac{1}{4}\left(e^{i \omega_{r} t-\omega_{i} t+i \alpha} \Delta p^{\dagger}+e^{-i \omega_{r} t-\omega_{i} t-i \alpha} \Delta p^{\dagger *}\right)\right. \\
& \left.\times \frac{1}{\bar{\rho}} \frac{\partial}{\partial t}\left(e^{i \omega_{r} t-\omega_{i} t+i \alpha} \Delta \rho^{\dagger}+e^{-i \omega_{r} t-\omega_{i} t-i \alpha} \Delta \rho^{\dagger *}\right)\right] \\
& \Leftrightarrow \frac{\partial}{\partial t}\left\{\int d V \left[\frac{1}{2} \bar{\rho}\left(e^{-2 \omega_{i} t}\left(\omega_{r}^{2}+\omega_{i}^{2}\right) \xi^{\dagger} \xi^{\dagger *}\right)\right.\right. \\
& \left.\left.+\left(\frac{1}{\bar{\rho}} \nabla \bar{p} \nabla \bar{\rho}-\nabla^{2} \bar{p}\right) \frac{1}{2}\left(e^{-2 \omega_{i} t} \xi^{\dagger} \xi^{\dagger *}\right)\right]\right\} \\
& =\int d V\left[-\frac{1}{\bar{\rho}} \frac{1}{4} e^{-2 \omega_{i} t}\left(\left(-i \omega_{r}-\omega_{i}\right) \Delta p^{\dagger} \Delta \rho^{\dagger *}+\left(i \omega_{r}-\omega_{i}\right) \Delta p^{\dagger *} \Delta \rho^{\dagger}\right)\right] \\
& \Leftrightarrow-\omega_{i} \int d V\left[\frac{1}{2} \bar{\rho}\left(\omega_{r}^{2}+\omega_{i}^{2}\right)+\frac{1}{2} \frac{1}{\bar{\rho}}\left(\nabla \bar{p} \nabla \bar{\rho}-\nabla^{2} \bar{p}\right)\right] \xi^{\dagger} \xi^{\dagger *} \\
& =\int d V \frac{1}{\bar{\rho}} \frac{1}{4}\left[i \omega_{r}\left(\Delta p^{\dagger} \Delta \rho^{\dagger *}+\Delta p^{\dagger *} \Delta \rho^{\dagger}\right)\right] \\
& +\int d V \frac{1}{\bar{\rho}} \frac{1}{4}\left[\omega_{i}\left(\Delta p^{\dagger} \Delta \rho^{\dagger *}+\Delta p^{\dagger *} \Delta \rho^{\dagger}\right)\right]
\end{aligned}
$$




$$
\begin{aligned}
\Leftrightarrow \quad \omega_{i} \int & d V\left[\bar{\rho}\left(\omega_{r}^{2}+\omega_{i}^{2}\right)+\frac{1}{\bar{\rho}}\left(\nabla \bar{p} \nabla \bar{\rho}-\nabla^{2} \bar{p}\right)\right] \xi^{\dagger} \xi^{\dagger *} \\
& +\frac{1}{\bar{\rho}} \frac{1}{2}\left(\Delta p^{\dagger} \Delta \rho^{\dagger *}+\Delta p^{\dagger *} \Delta \rho^{\dagger}\right) \\
= & \omega_{r} \int d V \frac{1}{\bar{\rho}} \mathfrak{I} \mathfrak{m}\left(\Delta p^{\dagger} \Delta \rho^{\dagger *}\right)
\end{aligned}
$$

The integral on the right hand side of equation B.5 is one of the representations of the classical work integral. Provided, the integral on the left hand side and the pulsation frequency $\omega_{r}$ do not vanish we have

$$
W=\frac{\omega_{i}}{\omega_{r}} \propto \int d V \frac{1}{\bar{\rho}} \mathfrak{I} \mathfrak{m}\left(\Delta p^{\dagger} \Delta \rho^{\dagger *}\right)
$$

The sign of the integrand in equation B.6 indicates whether a region of the star has a driving or damping influence. The differential form of the work integral is given by

$$
\begin{aligned}
\frac{d W}{d r} & =\frac{d}{d r} \int d V \frac{1}{\bar{\rho}} \mathfrak{I m}\left(\Delta p^{\dagger} \Delta \rho^{\dagger *}\right) \\
& =4 \pi r^{2} \bar{p} \mathfrak{I m}\left(\frac{\Delta p^{\dagger}}{\bar{p}} \frac{\Delta \rho^{\dagger *}}{\bar{\rho}}\right)
\end{aligned}
$$

Equation B.7 can be compared to equation C8 in Baker \& Kippenhahn (1962) by switching to the independent variable $\log \bar{p}$. This yields

$$
\begin{aligned}
\frac{d W}{d \log \bar{p}} & =\frac{d W}{d r} \frac{d r}{d \log \bar{p}} \\
& =4 \pi r^{2} \bar{p} \mathfrak{I m}\left(\frac{\Delta p^{\dagger}}{\bar{p}} \frac{\Delta \rho^{\dagger *}}{\bar{\rho}}\right) \frac{d r}{d \log \bar{p}} \\
& =4 \pi r^{2} \bar{p} \mathfrak{I m}\left(\frac{\Delta p^{\dagger *}}{\bar{p}} \frac{\Delta \rho^{\dagger}}{\bar{\rho}}\right) \frac{\bar{r}^{2} \bar{p}}{G M_{r} \bar{\rho}} \\
& =4 \pi \frac{\bar{p}^{2} \bar{r}^{4}}{G M_{r} \bar{\rho}} \mathfrak{I m}\left(\frac{\Delta p^{\dagger *}}{\bar{p}} \frac{\Delta \rho^{\dagger}}{\bar{\rho}}\right)
\end{aligned}
$$

whereas the result reported in Baker \& Kippenhahn (1962) is

$$
\frac{d W}{d \log \bar{p}}=4 \pi^{2} \frac{\bar{p}^{2} \bar{r}^{4}}{G M_{r} \bar{\rho}} \mathfrak{I m}\left(\frac{\Delta p^{\dagger *}}{\bar{p}} \frac{\Delta \rho^{\dagger}}{\bar{\rho}}\right)
$$

The difference by a factor $\pi$ is not relevant, since due to the ambiguity in the eigenfunction the differential work integral is determined only up to a real factor anyway. 


\section{Lebenslauf}

Name: Matthias Grott

Geboren: 11.08.1973 in Hamburg

Eltern: Uwe und Antje Grott

Staatsangehörigkeit: deutsch

Familienstand: ledig

1980 - $1984 \quad$ Grundschule Seth

$1984-1993$

Juni 1993

Juli 1993 - Sept. 1994

Okt. 1994 - Juli 1996

Juli 1996

Okt. 1996 - Juni 1997

Sept.1997 - Juli 2000

Jan. 1999 - Juli 2000

Juli 2000

Sept.2000 - Juli 2003

Abitur

Zivildienst
Dahlmannschule Bad Segeberg

Studium der Physik, Universität Göttingen.

Vordiplom in Physik, Note "gut"

Studium der Physik, University of Edinburgh

Studium der Physik, Universität Göttingen

Diplomarbeit mit dem Titel

"s-Produkt Zerlegung beschränkter Bosefelder

in 1+1 Dimensionen"

im Institut für Theoretische Physik

Betreuer: Prof. Dr. Karl-Henning Rehren

Diplom in Physik, Note "sehr gut"

Doktorarbeit mit dem Titel

"On the evolution and simulation of strange-mode instabilities"

an der Universitäts Sternwarte in Göttingen

Betreuer: Prof. Dr. Wolfgang Glatzel

Stipendium des Graduiertenkollegs

"Strömungsinstabilitäten und Turbulenz" 
